TRANSACTIONS OF THE

AMERICAN MATHEMATICAL SOCIETY

Volume 357, Number 6, Pages 2389-2444

S 0002-9947(04)03576-7

Article electronically published on October 28, 2004

\title{
CHARACTERISTIC SUBSURFACES AND DEHN FILLING
}

\author{
STEVE BOYER, MARC CULLER, PETER B. SHALEN, AND XINGRU ZHANG
}

\begin{abstract}
Let $M$ be a simple knot manifold. Using the characteristic submanifold theory and the combinatorics of graphs in surfaces, we develop a method for bounding the distance between the boundary slope of an essential surface in $M$ which is not a fiber or a semi-fiber, and the boundary slope of a certain type of singular surface. Applications include bounds on the distances between exceptional Dehn surgery slopes. It is shown that if the fundamental group of $M(\alpha)$ has no non-abelian free subgroup, and if $M(\beta)$ is a reducible manifold which is not homeomorphic to $S^{1} \times S^{2}$ or $P^{3} \# P^{3}$, then $\Delta(\alpha, \beta) \leq 5$. Under the same condition on $M(\beta)$, it is shown that if $M(\alpha)$ is Seifert fibered, then $\Delta(\alpha, \beta) \leq 6$. Moreover, in the latter situation, character variety techniques are used to characterize the topological types of $M(\alpha)$ and $M(\beta)$ in case the bound of 6 is attained.
\end{abstract}

\section{INTRODUCTION}

Many results in the theory of Dehn surgery (see [Go1]) assert that if $M$ is a compact, orientable, atoroidal, irreducible 3-manifold whose boundary is an incompressible torus, and if two Dehn fillings $M(\alpha)$ and $M(\beta)$ have specified properties, then the distance $\Delta(\alpha, \beta)$ of the slopes $\alpha$ and $\beta$ is bounded by a suitable constant. (The reader is referred to the body of this paper for the definition of "slope" and "distance", as well as for precise versions of many definitions, statements and proofs that are hinted at in this Introduction.)

In this paper, we define a closed 3-manifold to be very small if its fundamental group has no non-abelian free subgroup. The motivating result of the paper, Corollary 7.4.4, asserts that if $M(\alpha)$ is very small and $M(\beta)$ is a reducible manifold other than $S^{2} \times S^{1}$ or $P^{3} \# P^{3}$, then $\Delta(\alpha, \beta) \leq 5$. This follows from the following stronger result which deals with essential planar surfaces in $M$ which are not semi-fibers (see $1.3)$.

Corollary 7.4.3. Let $M$ be a simple knot manifold, and let $F \subset M$ be an essential planar surface with boundary slope $\beta$ which is not a semi-fiber. Let $\alpha$ be a slope in $\partial M$. If $M(\alpha)$ is very small, or more generally if $F \subset M \subset M(\alpha)$ is not $\pi_{1}$-injective in $M(\alpha)$, then $\Delta(\alpha, \beta) \leq 5$.

Received by the editors December 6, 2002 and, in revised form, December 2, 2003.

2000 Mathematics Subject Classification. Primary 57M25, 57M50, 57M99.

The first author was partially supported by NSERC grant OGP0009446 and FCAR grant ER-68657.

The second and third authors were partially supported by NSF grant DMS 0204142.

The fourth author was partially supported by NSF grant DMS 0204428 . 
Corollary 6.2.3 gives a qualitatively similar conclusion when $M(\alpha)$ is very small and $\beta$ is the boundary slope of an essential bounded surface $F$ of arbitrary genus which is not a semi-fiber. Here the upper bound for $\Delta(\alpha, \beta)$ is $15+(20 g-15) / m$, where $g$ is the genus of $F$ and $m$ is the number of components of $\partial F$. Note that, while this result does provide an upper bound in the case where $F$ is planar, the bound given by Corollary 7.4.3 is much stronger. In a follow-up paper we will examine the case where $g=1$ and show that in this situation, the bounds obtained in Corollary 6.2.3, and those described in the results mentioned below, can be significantly improved.

The proofs of these results begin with the observation that a bounded essential surface $F$ with boundary slope $\beta$ may be regarded as a non-properly embedded surface of negative Euler characteristic in $M(\alpha)$, and that if $M(\alpha)$ is very small, then the inclusion homomorphism from $\pi_{1}(F)$ to $\pi_{1}(M(\alpha))$ cannot be injective. From this one can deduce that there is a map of a disk into $M(\alpha)$ which maps the boundary of the disk into $M-F$ but cannot be homotoped rel boundary into $M-F$. After normalizing such a map and restricting it to the inverse image of $M$, one obtains a "singular surface" in $M$, "well positioned" with respect to $F$; according to the precise definitions given in Section 2, such a singular surface is defined by a map $h$ of a surface $S$ into $M$ having certain properties. In the case we are discussing here, $S$ is planar, and each component of $\partial S$ is either mapped into $M-F$ by $h$, or mapped homeomorphically onto a curve in $\partial M$ of slope $\alpha$. This last property is expressed by saying that the singular surface has boundary slope $\alpha$.

The main results of the paper, Theorems 6.2.2 and 7.4.2, give bounds on the distance between two slopes $\alpha$ and $\beta$ in terms of the data involving an essential surface $F$ in $M$ which is not a semi-fiber and has boundary slope $\beta$, and a singular surface which is well positioned with respect to $F$ and has boundary slope $\alpha$. Applying this in the case of a planar singular surface we obtain such results as Corollaries 6.2.3 and 7.4.3.

By applying Theorems 6.2.2 and 7.4.2 to other kinds of singular surfaces, we obtain different kinds of information about boundary slopes and Dehn filling. For instance we prove:

Corollary 7.4.5. Let $M$ be a simple knot manifold, and let $F \subset M$ be an essential planar surface with boundary slope $\beta$ which is not a semi-fiber. Let $\alpha$ be a slope in $\partial M$. If $M(\alpha)$ is a Seifert fibered space or if there exists a $\pi_{1}$-injective map from $S^{1} \times S^{1}$ to $M(\alpha)$, then $\Delta(\alpha, \beta) \leq 6$.

This implies Corollary 7.4.6, which asserts that if $M(\alpha)$ is a Seifert fibered space and $M(\beta)$ is a reducible manifold other than $S^{2} \times S^{1}$ or $P^{3} \# P^{3}$, then $\Delta(\alpha, \beta) \leq 6$. Corollary 7.4.5, like Corollary 7.4.3, has a high-genus analogue: Corollary 6.2.4 asserts that if $M(\alpha)$ is a Seifert fibered space and $\beta$ is the boundary slope of an essential surface $F$ in $M$ which is not a semi-fiber, then $\Delta(\alpha, \beta) \leq 18+(24 g-18) / m$, where $g$ is the genus of $F$ and $m$ is the number of its boundary components. These results are proved by observing that if $M(\alpha)$ is Seifert fibered, then either it is very small, in which case the conclusions follow from Corollaries 6.2.3 and 7.4.3, or it contains a $\pi_{1}$-injective singular torus. Such a torus can be used to construct a genus-1 singular surface in $M$ having boundary slope $\alpha$, to which Theorems 6.2.2 and 7.4.2 can be applied. 
Still another type of application of Theorems 6.2.2 and 7.4.2 can be obtained by observing that an essential surface in $M$ with boundary slope $\alpha$ is a special case of a singular surface with boundary slope $\alpha$. This leads to upper bounds for the distance between boundary slopes of two essential surfaces (not both semifibers) in terms of the genera and numbers of boundary components of the surfaces. Such bounds are given in Corollary 6.2.5 in the general case, and Corollary 7.4.7, which recovers a result of Gordon and Litherland [GLi Proposition 6.1] under the additional hypothesis that one of the surfaces is planar and is not a semifiber. Corollary 6.2.5, which is qualitatively similar to an unpublished result due to Cameron Gordon, strengthens a result due to Torisu [T], but in turn has been strengthened slightly by Agol $[\mathrm{A}]$, using results of Cao and Meyerhoff [CM].

The constructions of singular surfaces that are needed to pass from Theorems 6.2.2 and 7.4.2 to their various corollaries are given in detail in Section 2.

The statements of Theorems 6.2.2 and 7.4.2 involve an essential surface $F$ which is not a semi-fiber. We shall sketch the proofs under the simplifying assumption that $M-F$ has two components, whose closures we shall denote by $M_{F}^{+}$and $M_{F}^{-}$. The first step in the proofs, which is carried out in Section 3, involves graph-theoretical arguments. Suppose that we are given an essential surface $F \subset M$ with boundary slope $\beta$ and a singular surface which is well positioned with respect to $F$ and has boundary slope $\alpha$. Such a singular surface is defined by a certain kind of map $h$ of a compact 2-manifold $S$ into $M$. We obtain a surface $\hat{S}$ from $S$ by identifying certain components of $\partial S$ to points, and the images of the arc components of $h^{-1}(S)$ are the edges of a graph $G \subset \hat{S}$. Each vertex of $G$ has valence $m \Delta(\alpha, \beta)$, where $m$ is the number of boundary components of $F$. By using certain non-degeneracy properties of $G$ we find a family of parallel edges in $G$ whose size is bounded below in terms of topological data about $F$ and the valence $m \Delta(\alpha, \beta)$.

A parallel family of edges in $G$ gives 3-dimensional information about how the essential surface $F$ sits in $M$. While the edges of $G$ do not map to properly embedded arcs in $F$, each edge of $G$ does give rise to an essential path in $(F, \partial F)$, which can be extended to a map of a "pair of glasses" (see Figure 3.3.1) into $F$ that maps the rims homeomorphically to components of $\partial F$. A parallel family of $k+1$ edges in $G$ defines a sequence of $k+1$ such "singular pairs of glasses" and $k$ essential homotopies in $M_{F}^{+}$and $M_{F}^{-}$between the successive pairs of glasses in the sequence. (Under the homotopies, the images of the rims of the glasses stay in $\partial M$.) Furthermore, these homotopies alternate strictly between homotopies in $M_{F}^{+}$and homotopies in $M_{F}^{-}$. According to the precise definition given in Section 3 such a sequence of homotopies determines a reduced homotopy of length $k$. The graph-theoretical arguments that we have sketched here are used in Section 3 to show that upper bounds for the length of a reduced homotopy of singular pairs of glasses in $M$ imply theorems of the type of 6.2.2 and 7.4.2. The rest of the paper is devoted to obtaining such bounds for the lengths of reduced homotopies, in the more general context of a map of a polyhedron into $M$ which is "large" in the sense that the induced homomorphism of fundamental groups has a non-abelian image.

A reduced homotopy of length 1 is by definition an essential homotopy in $M_{F}^{+}$ or $M_{F}^{-}$whose time-0 and time-1 maps are maps of the domain into $F$. We are interested in reduced homotopies whose time- 0 maps are large. Such homotopies can be understood in terms of the characteristic submanifold theory ([JS], [Jo] $)$. This theory provides a (possibly disconnected) 2-manifold $\Phi^{ \pm} \subset M_{F}^{ \pm}$, which is 
"large" in the sense that the fundamental group of each component of $\Phi^{ \pm}$is nonabelian and maps injectively into $\pi_{1}(F)$. Any large map of a polyhedron into $F$ which is the time-0 map of an essential homotopy in $M_{F}^{ \pm}$is homotopic in $F$ to a map into $\Phi^{ \pm}$. Furthermore, the identity map of $\Phi^{ \pm}$is itself the time-0 map of an essential homotopy in $M_{F}^{ \pm}$.

In Section 5 we generalize this to reduced homotopies of length $k$ : we define large 2-dimensional submanifolds $\Phi_{k}^{ \pm}$of $F$ for $k=1,2, \ldots$ If a large map $f$ of a polyhedron into $F$ is the time- 0 map of a length- $k$ reduced homotopy in $M$ which "begins" in $M_{F}^{+}$(or $M_{F}^{-}$), then $f$ is homotopic in $F$ to a map into $\Phi_{k}^{+}$(respectively $\Phi_{k}^{-}$). Furthermore, the identity map of $\Phi_{k}^{ \pm}$is itself the time-0 map of a reduced homotopy in $M$ which begins in $M_{F}^{ \pm}$. The $\Phi_{k}^{ \pm}$for $k>1$ are defined inductively, using the notion of an essential intersection of subsurfaces of $F$, which is presented in [Ja]. In Section 4 we give a self-contained account of a version of the theory of essential intersections that is adapted to the study of large subsurfaces.

The $\Phi_{k}^{ \pm}$give a natural tool for bounding the length of a reduced homotopy: if $n$ is a positive integer such that $\Phi_{n}^{+}=\Phi_{n}^{-}=\emptyset$, it is clear that any reduced homotopy with large time-0 map has length $<n$. (Of course there can be no such bound in the case that $F$ is a semi-fiber.)

The $\Phi_{k}^{ \pm}$may be taken to be nested: $\Phi_{1}^{+} \supset \Phi_{2}^{+} \supset \ldots$, and similarly for the $\Phi_{k}^{-}$. A crucial step in the argument is provided by Proposition 5.3.9, which asserts that when $F$ is not a semi-fiber, and if $\Phi_{k}^{+}$(say) is non-empty for a given $k$, then $\Phi_{k+2}^{+}$is not isotopic to $\Phi_{k}^{+}$. Hence in the sequence $\Phi_{1}^{+} \supset \Phi_{3}^{+} \supset \Phi_{5}^{+} \supset \ldots$, the successive subsurfaces are always non-isotopic until one of them becomes empty. This means that to bound the length of a reduced homotopy whose time-0 map is large, it suffices to bound the length of a nested sequence of subsurfaces of $F$ in which successive subsurfaces are non-isotopic. This is a matter of elementary surface topology, and the bound can be improved by a factor of 2 using Corollary 5.3.8, which asserts that for odd $k$ the $\Phi_{k}^{ \pm}$all have even Euler characteristic. This leads to Theorem 5.4.1, which gives a bound of $8 g+3 m-8$ for the length of a reduced homotopy with a large time- 0 map, where $g$ is the genus of $F$ and $m$, as above, denotes the number of its boundary components.

While Theorem 5.4.1 is significant for general large maps, it is far from optimal for the case of singular pairs of glasses arising from essential paths. In Section 6 we introduce a variant of $\Phi_{k}^{ \pm}$which we denote by $\dot{\Phi}_{k}^{ \pm}$; it is simply the union of the "outer components" of $\Phi_{k}^{ \pm}$, i.e. those components which have at least one boundary component which is homotopic to a component of $\partial F$. If a singular pair of glasses $f$ arising from an essential path in $F$ is the time- 0 map of a length- $k$ reduced homotopy in $M$ which begins in $M_{F}^{+}$(or $M_{F}^{-}$), then $f$ is homotopic in $F$ to a map into $\dot{\Phi}_{k}^{+}$(respectively $\dot{\Phi}_{k}^{-}$). In Section 6 , under the assumption that there exists a reduced homotopy of length $m$ whose time- 0 map is an essential path, we establish analogues for the $\dot{\Phi}_{k}^{ \pm}$of all the properties of the $\Phi_{k}^{ \pm}$that are established in Section 4, including Corollary 6.1.10 and Proposition 6.1.11 which are the analogues of Corollary 5.3.8 and Proposition 5.3.9. By definition the $\dot{\Phi}_{k}^{ \pm}$ have the additional property that they are outer subsurfaces, in the sense that all their components are outer components. Hence to bound the length of a reduced homotopy whose time-0 map is an essential path, it suffices to bound the length of a nested sequence of outer subsurfaces of $F$ in which successive subsurfaces are non-isotopic. The restriction to outer subsurfaces turns out to improve the bound, 
almost by another factor of 2 . The upshot is Theorem 6.2.1, which gives a bound of $4 g+3 m-4$ for the length of a reduced homotopy whose time- 0 map is an essential path, where $g$ and $m$ are defined as above. Theorem 6.2 .2 is proved by combining Theorem 6.2.1 with the results of Section 2.

In order to prove Theorem 7.4.2 we need to improve the conclusion of Theorem 6.2.1 in the special case where $F$ is planar, i.e. $g=0$.

Theorem 7.4.1. Let $F$ be an essential planar surface in a simple knot manifold $M$. Suppose that $F$ is not a semi-fiber. Set $m=|\partial F|$ and let $H$ be any reduced homotopy in the pair $(M, F)$ such that $H_{0}$ is an essential path in $F$ and $H_{t}(\partial I) \subset \partial M$ for each $t \in I$. Then the length of $H$ is at most $m-1$.

The proof formally proceeds by contradiction, beginning with the assumption that there is a length- $m$ reduced homotopy whose time- 0 map is an essential path, and applying some machinery that is set up in Section 7. We shall sketch this machinery in the case where $F$ is planar, although many of the results of Section 7 are stated more generally. We introduce yet another variant of $\Phi_{k}^{ \pm}$which we denote by $\breve{\Phi}_{k}^{ \pm}$. It differs from $\dot{\Phi}_{k}^{ \pm}$in that it contains $\partial F$, but may have annular components. Under the assumption that there is a length- $m$ reduced homotopy whose time-0 map is an essential path, we again obtain analogues for the $\breve{\Phi}_{k}^{ \pm}$of the properties that are established in the preceding sections for the $\Phi_{k}^{ \pm}$and the $\dot{\Phi}_{k}^{ \pm}$, even though the surfaces $\breve{\Phi}_{k}^{ \pm}$need not be large. In Proposition 7.2.10, which is the analogue of Propositions 5.3.9 and 6.1.11, the analogue of the conditions $\Phi_{k}^{ \pm}=\emptyset$ or $\dot{\Phi}_{k}^{ \pm}=\emptyset$ is that $\breve{\Phi}_{k}^{ \pm}$is a regular neighborhood of $\partial F$, which is in fact equivalent to the condition $\dot{\Phi}_{k}^{ \pm}=\emptyset$.

The planarity of $F$ implies that if $F$ is not a semi-fiber, then some component of $\breve{\Phi}_{1}^{ \pm}$is tight in the sense that its frontier in $F$ is a single simple closed curve. We define the size of a tight component of $\breve{\Phi}_{k}^{ \pm}$to be the number of components of $\partial F$ that it contains. We call a component of $\breve{\Phi}_{1}^{+}$or $\breve{\Phi}_{1}^{-}$very tight if its size is at most the minimum size of any tight component of $\breve{\Phi}_{1}^{+}$or $\breve{\Phi}_{1}^{-}$. We may assume by symmetry that $\breve{\Phi}_{1}^{+}$has a very tight component. Lemma 7.3.1, the proof of which is based on the same ideas as that of Corollary 5.3.8, implies that the number $\left|V T\left(\breve{\Phi}_{k}^{+}\right)\right|$of very tight components of $\breve{\Phi}_{k}^{+}$is always even. A key step in proving Theorem 7.4.1 is Lemma 7.3.7, which implies that if $F$ is not a semi-fiber, then increasing $k$ by 2 always strictly increases $\left|V T\left(\breve{\Phi}_{k}^{+}\right)\right|$, unless $\breve{\Phi}_{k}^{+}$is already a regular neighborhood of $\partial F$. From this it is not hard to deduce (cf. Proposition 7.3.8) that $\breve{\Phi}_{m-1}^{+}$is a regular neighborhood of $\partial F$; this in turn easily implies that there is no length- $m$ reduced homotopy whose time- 0 map is an essential path, a contradiction which completes the proof of Theorem 7.4.1.

In Section 8 we further investigate the situation where $M$ is a simple knot manifold and $\alpha$ and $\beta$ are slopes such that $M(\alpha)$ is Seifert fibered while $M(\beta)$ is reducible. In Proposition 8.4 we establish restrictions on which Seifert fibered spaces can arise in this situation when $\Delta(\alpha, \beta)>3$. The proof begins by applying Corollary 7.4.6 to deduce that $\Delta(\alpha, \beta)$ is equal to 4,5 or 6 . We then use the $P S L_{2}(\mathbb{C})$ character variety together with some observations from algebraic number theory to describe the Seifert fibered structure on $M(\alpha)$. For instance we prove:

Corollary 8.5. Let $M$ be a simple knot manifold and fix slopes $\alpha$ and $\beta$ on $\partial M$. If $M(\beta)$ is reducible, though not $S^{1} \times S^{2}$ or $P^{3} \# P^{3}$, and $M(\alpha)$ is a Seifert fibered 
space, then $\Delta(\alpha, \beta) \leq 5$ unless perhaps $M(\beta) \cong P^{3} \# L(p, q)$ and $M(\alpha)$ is a small Seifert manifold with base orbifold $S^{2}(a, b, c)$, where $(a, b, c)$ is a hyperbolic triple and 6 divides $l c m(a, b, c)$.

Similar methods lead to restrictions, in Proposition 8.7, of the possible Seifert fibrations of $M(\alpha)$ when $M(\beta)$ is a Seifert fibered space containing an (embedded) incompressible torus and $5<\Delta(\alpha, \beta) \leq 10$. The method of proof is similar to that used in Proposition 8.4, except that the role of Corollary 7.4.6 is played by a theorem due to Agol $[\underline{\mathrm{A}}]$ and Lackenby $[\mathrm{La}$ which implies that $\Delta(\alpha, \beta) \leq 10$ in this situation.

This paper had its origins in unpublished work done by M. Culler, C. MacA. Gordon and P. B. Shalen in 1984. This work established a preliminary version of the Cyclic Surgery Theorem [CGLS], giving an upper bound of 5 for the distance between two cyclic filling slopes for a simple knot manifold, which was afterwards superseded by the bound of 1 established in CGLS. The proof used character variety techniques to reduce the result to a complicated topological statement, which was in turn proved by combining the graph-theoretical construction of Section 3 and the use of the subsurfaces $\Phi_{k}^{ \pm}$in the same way as is done in the proof of Theorem 6.2.2. The techniques that were then available could have produced a result qualitatively similar to Theorem 6.2 .2 , but much weaker.

Independently and more recently, techniques similar to ours have been used in a related context by Cooper and Long [CL] and $\mathrm{Li}[\mathrm{Li}]$. While our main results bound $\Delta(\alpha, \beta)$ under the assumption that $\beta$ is a boundary slope and $\pi_{1}(M(\alpha))$ does not contain a non-abelian free group, the results in $\mathrm{CL}$ ] and [i] imply a weaker bound for $\Delta(\alpha, \beta)$ under the weaker assumption that $\beta$ is a boundary slope and $\pi_{1}(M(\alpha))$ does not contain the fundamental group of a closed surface of genus $>1$. Li proves a result which is qualitatively similar to our Theorem 6.2.1, but with a bound of $6 g+4 m-6$ where Theorem 6.2.1 provides the stronger bound of $4 g+3 m-4$.

We are indebted to Cameron Gordon for his role in the development of the ideas in this paper.

\section{TERMinOLOGY AND NOTATION}

We describe here various notational conventions that will be used throughout the paper.

1.1. If $X$ is a topological space, $|X|$ will denote the number of components of $X$. A (continuous) map $f: X \rightarrow Y$ of topological spaces will be called $\pi_{1}$-injective if for each $x_{0} \in X$, the homomorphism $f_{\sharp}: \pi_{1}\left(X ; x_{0}\right) \rightarrow \pi_{1}\left(Y ; f\left(x_{0}\right)\right)$ is injective. A subset $A$ of a space $Y$ will be called $\pi_{1}$-injective if the inclusion map $A \rightarrow Y$ is $\pi_{1}$-injective.

A homotopy with domain $X$ and target $Y$ is a map $H: X \times I \rightarrow Y$. For each $t \in[0,1]$ we define $H_{t}: X \rightarrow Y$ by $H_{t}(x)=H(x, t)$. We shall sometimes refer to $H_{t}$ as the time-t map of $H$.

Let $H^{1}, \ldots, H^{n}$ be homotopies with domain $X$ and target $Y$. A homotopy $H$ with domain $X$ and target $Y$ will be said to be a composition of $H^{1}, \ldots, H^{n}$ if there exist numbers $0=x_{0}<x_{1} \cdots<x_{n}=1$ and monotone increasing linear homeomorphisms $\alpha_{i}:\left[x_{i-1}, x_{i}\right] \rightarrow[0,1]$ such that $H(x, t)=H^{i}\left(x, \alpha_{i}(t)\right)$ whenever $t \in\left[x_{i-1}, x_{i}\right]$. 
We shall say that a map $f: X \rightarrow Y$ between spaces is homotopic into a subset $B$ of $Y$ if $f$ is homotopic to a map $g: X \rightarrow Y$ for which $g(X) \subset B$. A subset $A$ of a space $Y$ is said to be homotopic into a subset $B$ of $Y$ if the inclusion map from $A$ to $Y$ is homotopic into $B$.

Let $f:(X, Y) \rightarrow(Z, W)$ be a map of topological pairs, where $Z$ is a connected $n$-manifold and $W \subset \partial Z$ is an $(n-1)$-manifold. In the case where $X$ is pathwise connected, we shall say that $f$ is essential if it is $\pi_{1}$-injective as a map from $X$ to $Z$ and is not homotopic, as a map of pairs, to a map $f^{\prime}:(X, Y) \rightarrow(Z, W)$ where $f^{\prime}(X) \subset W$. In general we shall say that $f$ is essential if $X \neq \emptyset$ and $f$ restricts to an essential map from $(C, C \cap Y)$ to $(Z, W)$ for every component $C$ of $X$. A map $f$ from a space $X$ to a manifold $Z$ will be termed essential if $f:(X, \emptyset) \rightarrow(Z, \partial Z)$ is essential.

We will denote the unit interval $[0,1]$ by $I$. By an essential path in a surface $F$ with non-empty boundary we shall mean an essential map $f:(I, \partial I) \rightarrow(F, \partial F)$.

A manifold $M$ is said to be orientable if all of its components are orientable. An orientation of $M$ is defined by a choosing an orientation for each component; a manifold $M$ is said to be oriented if an orientation of $M$ has been fixed. Every codimension 0 submanifold of an oriented manifold $M$ inherits an orientation.

Suppose that $A$ is a codimension 0 submanifold of a manifold $M$ and that $h$ : $A \rightarrow M$ is an embedding. We say that $h$ preserves orientation if it carries the orientation of $A$ inherited from $M$ to the orientation of $h(A)$ inherited from $M$; we say that $h$ reverses orientation if it carries the orientation of $A$ inherited from $M$ to the orientation of $h(A)$ which is the opposite of that inherited from $M$. In general, if $A$ is disconnected, there may exist embeddings which neither preserve nor reverse orientation.

If $S$ is a compact surface, then $\chi(S)$ denotes the Euler characteristic of $S$ and $\operatorname{genus}(S)$ denotes the total genus of $S$, i.e. the sum of the genera of the components of $S$.

1.2. A compact orientable 3-manifold $M$ is said to be irreducible if it is connected and every 2-sphere in $M$ bounds a ball. A compact orientable 3-manifold $M$ is said to be boundary irreducible if it is connected and for any properly embedded disk $D$ in $M$ the curve $\partial D$ bounds a disk in $\partial M$.

Let $M$ be a compact, orientable, irreducible 3-manifold, and let $Q \subset \partial M$ be a compact $\pi_{1}$-injective surface. We define an essential surface in $(M, Q)$ to be a compact surface $F$ in $M$ such that (i) $\partial F=F \cap \partial M \subset Q$, and (ii) the inclusion map $(F, \partial F) \rightarrow(Z, Q)$ is essential. Note that condition (ii) is equivalent to the condition that $F$ is incompressible and not parallel to a subsurface of $Q$. By an essential surface in a 3 -manifold $M$ we mean an essential surface in $(M, \partial M)$.

Let $M$ be a compact, orientable, irreducible 3-manifold and let $Q$ be a $\pi_{1^{-}}$ injective subsurface of $\partial M$. We will say that the pair $(M, Q)$ is acylindrical if there does not exist any essential map from $\left(S^{1} \times I, S^{1} \times \partial I\right)$ to $(M, \partial M)$ which sends $S^{1} \times \partial I$ into $Q$. By the Annulus Theorem [JS IV.3.1], $(M, Q)$ is acylindrical if and only if there is no essential annulus in $(M, Q)$.

We will say that a compact 3-manifold $M$ is atoroidal if there exists no essential map from $\left(S^{1} \times S^{1}, \emptyset\right)$ to $(M, \partial M)$.

A closed orientable 3-manifold will be said to be very small if its fundamental group contains no non-abelian free subgroup. 
1.3. Given a compact orientable 3 -manifold $M$ and a connected, properly embedded, transversely oriented surface $F$ in $M$, we will implicitly fix a regular neighborhood $N(F)$ of $F$ and a homeomorphism $f_{F}$ from $F \times[-1,1]$ to $N(F)$ which sends the standard orientation of $[-1,1]$ to the transverse orientation of $F$ and maps $F \times\{0\}$ to $F$. The sets $f_{F}(F \times(-1,0])$ and $f_{F}(F \times[0,1))$ will be denoted $N_{-}(F)$ and $N_{+}(F)$, respectively.

We will use the notation $M_{F}$ to denote the compact manifold

$$
M-f_{F}(F \times(-1 / 2,1 / 2)) .
$$

The boundary of $M_{F}$ contains the two subsurfaces $F \times\{-1 / 2\}$ and $F \times\{1 / 2\}$ which will be denoted $F_{-}$and $F_{+}$, respectively. By restricting the projection map $N(F) \rightarrow F$ we obtain two standard homeomorphisms $i_{-}: F_{-} \rightarrow F$ and $i_{+}: F_{+} \rightarrow$ $F$. Whenever it is convenient to do so we will identify the manifold $M$ with the quotient of $M_{F}$ obtained by gluing $F_{-}$to $F_{+}$via the homeomorphism $i_{+}^{-1} \circ i_{-}$.

The surface $F$ will be called a semi-fiber in $M$ if the pair $\left(M_{F}, F_{-} \cup F_{+}\right)$is an $I$-pair in the sense of [Ja]; that is, if there is an $I$-bundle $E$ over a surface and a homeomorphism $h: M_{F} \rightarrow E$ such that $h\left(F_{-} \cup F_{+}\right)$is the $\partial I$-bundle associated to $E$. (Note that $E$ may be either a trivial $I$-bundle over a connected orientable surface, or a twisted $I$-bundle over a non-orientable surface of two components.)

1.4. Simple knot manifolds and slopes. We will say that a compact connected orientable 3 -manifold $M$ is simple provided that (1) $M$ is irreducible and boundary irreducible, (2) $M$ contains no essential surface of Euler characteristic 0, and (3) $M$ is not Seifert-fibered. If in addition the boundary of $M$ is a torus we will say that $M$ is a simple knot manifold.

If $M$ is a simple knot manifold, then an unoriented isotopy class of homotopically non-trivial simple closed curves on $\partial M$ will be called a slope. We will write $\Delta(\alpha, \beta)$ for the geometric intersection number of two slopes $\alpha$ and $\beta$. We will denote by $M(\alpha)$ the Dehn filling of $M$ determined by $\alpha$. If $F$ is a bounded essential surface in $M$, then the boundary curves of $F$ all have the same slope, which will be called the boundary slope of $F$. A slope will be called a boundary slope if it is the boundary slope of some essential surface. A slope will be called a strict boundary slope if it is the boundary slope of some essential surface which is not a semi-fiber in $M$.

\section{Singular SURFACES}

Let $M$ be a simple knot manifold. By a singular surface in $M$ we will mean a triple $(S, X, h)$, where $S$ is a compact, connected, orientable surface, $X$ is a nonempty union of components of $\partial S$, and $h:(S, X) \rightarrow(M, \partial M)$ is a map of pairs such that (i) $h(S-X) \subset$ int $M$ and (ii) $h$ maps the components of $X$ homeomorphically onto disjoint, homotopically non-trivial simple closed curves in $\partial M$.

If $(S, X, h)$ is a singular surface in $M$, the components of $h(X)$ are all simple closed curves with the same slope, which we shall call the boundary slope of $(S, X, h)$.

We shall say that two simple closed curves $\gamma$ and $\gamma^{\prime}$ on a torus $T$ are in standard position if there is a covering map $p: \mathbb{R}^{2} \rightarrow T$ for which $p^{-1}(\gamma)$ and $p^{-1}\left(\gamma^{\prime}\right)$ are Euclidean lines.

Let $F$ be a bounded essential surface in a simple knot manifold $M$. A singular surface $(S, X, h)$ will be said to be well positioned with respect to $F$ if (i) $h$ is transverse to $F$, (ii) $h(\partial S-X) \cap F=\emptyset$, (iii) $h(X)$ is in standard position with respect to $\partial F$, and (iv) each component of $h^{-1}(F)$ is mapped by $h$ to an essential 
path or to a homotopically non-trivial (possibly singular) closed curve in $F$. It follows from (ii) that the arc components of $h^{-1}(F)$ have their endpoints in $X$. Note that (iv) implies in particular that no simple closed curve component of $h^{-1}(F)$ bounds a disk in $S$, and that no arc component of $h^{-1}(F)$ is parallel in $S$ to an arc in $X$.

In this section we shall give several ways of constructing singular surfaces that are well positioned with respect to a given bounded essential surface in a simple knot manifold.

Proposition 2.1. Suppose that $F$ and $S$ are bounded essential surfaces in a simple knot manifold $M$. Then the inclusion map from $S$ to $M$ is isotopic to an embedding $h: S \rightarrow M$ such that the singular surface $(S, \partial S, h)$ is well positioned with respect to $F$.

Proof. After an isotopy we may assume that $S$ and $F$ meet transversely and that $\partial S$ is in standard position with respect to $F$. We claim that every arc component of $S \cap F$ is essential in $F$. If this is not the case, then there is a disk $D \subset F$ whose frontier in $F$ is an arc $A$ such that $A=D \cap S$. Since $\partial M$ is a torus, the essential surface $S$ is boundary-incompressible; hence $A$ is the frontier in $S$ of a disk $E \subset S$. Now $D \cup E$ is a properly embedded disk in the simple knot manifold $M$ and is therefore parallel in $M$ to a disk $J \subset \partial M$. As $\partial J$ is made up of an arc in $\partial F$ and an arc in $\partial S$, we have a contradiction to standard position, and the claim is proved.

To prove the proposition it now suffices to show that if some component of $S \cap F$ is a homotopically trivial simple closed curve $C$ in $F$, then $S$ is isotopic rel boundary to a surface $S^{\prime}$ such that $\left|S^{\prime} \cap F\right|<|S \cap F|$. We may suppose $C$ to be chosen so that there is a disk $D \subset F$ such that $\partial D=C=D \cap S$. Since $S$ is essential, $C$ also bounds a disk $E \subset S$; since $M$ is irreducible the disks $D$ and $E$ are isotopic by an isotopy that fixes $C$. We may thus obtain the required surface $S^{\prime}$ by moving $(S-i n t E) \cup D$ into general position with respect to $F$.

Proposition 2.2. Let $M$ be a simple knot manifold. Let $F$ be a bounded essential surface in $M$, and let $\alpha$ be a slope on $\partial M$. Suppose that there exist a compact orientable surface $T$ and a $\pi_{1}$-injective map $f: T \rightarrow M(\alpha)$ such that

(1) $f(\partial T) \subset M-F \subset M \subset M(\alpha)$;

(2) there exists no map from $T$ to $M$ which agrees with $f$ on $\partial T$ and induces an injection from $\pi_{1}(T)$ to $\pi_{1}(M(\alpha))$.

Then there exists a singular surface $(S, X, h)$ in $M$ which has boundary slope $\alpha$ and is well positioned with respect to $F$. Moreover, we have genus $S=$ genus $T$, and $|\partial S-X|=|\partial T|$.

Proof. Let us write $M(\alpha)=M \cup V$, where $V$ is a solid torus and a meridian curve of $V$ is identified with a curve of slope $\alpha$ in $\partial M$.

By transversality and uniqueness of regular neighborhoods, there exists a map $h: T \rightarrow M(\alpha)$ such that

(i) $h$ is $\pi_{1}$-injective and agrees with $f$ on $\partial T$;

(ii) the components of $h^{-1}(V)$ are disks in the interior of $T$ which are mapped homeomorphically by $h$ to disjoint meridian disks of $V$, whose boundaries are all in standard position with respect to all of the components of $\partial F$;

(iii) each component of $h^{-1}(F)$ is a properly embedded 1-manifold in the bounded surface $h^{-1}(M)$. 
Define the complexity of a map $h$ satisfying conditions (i) - (iii) to be the ordered pair $(v(h), b(h))$, where $v(h)$ is the number of components of $h^{-1}(V)$ and $b(h)$ is the number of components of $h^{-1}(F)$. Note that hypothesis (2) and condition (i) imply that $v(h)$ must be strictly positive.

Among all maps satisfying (i) - (iii), we suppose $h$ to be chosen so that the complexity of $h^{-1}(F)$ is minimal with respect to lexicographical order.

We set $S=T-h^{-1}($ int $V)=h^{-1}(M)$ and $X=\partial S-\partial T=h^{-1}(\partial M)$. By (ii), the components of $h(X)$ are simple closed curves of slope $\alpha$.

We shall show that the map $h$ satisfies the following additional condition:

(iv) Each component of $h^{-1}(F)$ is mapped by $h$ to an essential path or to a homotopically non-trivial (possibly singular) closed curve in $F$.

It follows from the definitions that if $h$ satisfies (i)-(iv), then the triple $(S, X, h \mid S)$ is a singular surface, well positioned with respect to $F$ and having boundary slope $\alpha$. Furthermore, we have genus $S=$ genus $T$ and $|\partial S-X|=|\partial T|$, since $S$ was obtained from $T$ by removing a collection of disjoint disks bounded by $X$. Hence the proof of the proposition will be complete when we have shown that $h$ satisfies (iv).

To prove (iv), first suppose that some curve component $C$ of $h^{-1}(F)$ is mapped by $h$ to a homotopically trivial closed curve in $F$. Then, since $h$ is $\pi_{1}$-injective, the curve $C$ bounds a disk $D$ in $T$. Thus there is a map $h^{\prime}: T \rightarrow M(\alpha)$ which agrees with $h$ on the complement of $D$, and maps $D$ into $F$. Moving $h^{\prime}$ into general position would produce a map of lower complexity than $h$, giving a contradiction.

To complete the proof of (iv), we consider an arc component $A$ of $h^{-1}(F)$, and let $p, q \in X$ be the endpoints of $A$. We must show that the path $h(A)$ is essential in $F$.

It will be convenient to work in the smooth category for this argument. Fix an orientation on the manifold $M$ and give $\partial M$ the induced orientation. Given an ordered pair $\left(\gamma_{1}, \gamma_{2}\right)$ of oriented 1-manifolds on $\partial M$, intersecting transversely at a point, we define the sign of their intersection at that point to be the sign of the frame $\left(\mathbf{u}_{1}, \mathbf{u}_{2}\right)$ with respect to the orientation of $\partial M$, where $\mathbf{u}_{i}$ is a tangent vector to $\gamma_{i}$ which is positive with respect to its orientation.

Next we fix orientations of $F$ and $T$. The orientation of $T$ restricts to an orientation of $S$. Give the components of $\partial F$ and $X$ the orientations induced from those of $F$ and $S$, and then push the orientation of $X$ forward under $h$ to obtain orientations of the meridian curves that make up $h(X)$. The points $h(p)$ and $h(q)$ are transverse intersection points of the oriented 1-manifolds $\partial F$ and $h(X)$ on $\partial M$. A key step in the proof that $h(A)$ is an essential path is to show that the signs of these intersections are opposite.

The orientations of $M$ and $F$ define a transverse orientation of $F$. Because the map $h$ is transverse to $F$, we can pull back the transverse orientation of $F$ to a transverse orientation of $A$ in $S$. Let $\mathbf{u}_{p}$ and $\mathbf{u}_{q}$ be tangent vectors to $X$ at $p$ and $q$ which are positive with respect to the orientation of $X$. Let $\mathbf{v}_{p}$ and $\mathbf{v}_{q}$ be tangent vectors to $\partial F$ at the points $h(p)$ and $h(q)$ which are positive with respect to the orientation of $\partial F$. Let $\mathbf{w}_{p}$ and $\mathbf{w}_{q}$ be tangent vectors to $\partial M$ which are transverse to $\mathbf{v}_{p}$ and $\mathbf{v}_{q}$ and positive with respect to the transverse orientation of $F$. Observe first that, since $A$ is a properly embedded $\operatorname{arc}$ in $S$, the transverse orientation of $A$ is consistent with the orientation of $X$ at $p$ if and only if it is inconsistent with the orientation of $X$ at $q$. Second, observe that the signs of $\left(\mathbf{v}_{p}, \mathbf{w}_{p}\right)$ and $\left(\mathbf{v}_{q}, \mathbf{w}_{q}\right)$ 
agree. It follows that the signs of $\left(D h\left(\mathbf{u}_{p}\right), \mathbf{v}_{p}\right)$ and $\left(D h\left(\mathbf{u}_{q}\right), \mathbf{v}_{q}\right)$ are opposite. In other words, the signs of the intersections of $\partial F$ and $h(X)$ are opposite at $h(p)$ and $h(q)$.

Now assume that the path $h(A)$ is inessential in $F$. In particular the points $h(p)$ and $h(q)$ then lie on the same component of $\partial F$. But the signs of the intersections of $\partial F$ and $h(X)$ are opposite at $h(p)$ and $h(q)$, and by (ii) the components of $h(X)$ are all in standard position with respect to all the components of $\partial F$. It follows that $p$ and $q$ lie on distinct components $C_{1}$ and $C_{2}$ of $X$, and that the orientations inherited from $h(X)$ by the meridian curves $h\left(C_{1}\right)$ and $h\left(C_{2}\right)$ are opposite on the torus $\partial M$. Let $D_{1}, D_{2} \subset T$ be the disk components of $h^{-1}(V)$ bounded by $C_{1}$ and $C_{2}$, and define $D$ to be a regular neighborhood of $D_{1} \cup A \cup D_{2}$ in $T$. Then $h(\partial D)$ is homotopic in $M$ to a (possibly singular) closed curve on the torus $\partial M$ which is the composition of conjugates of the oppositely oriented meridian curves $h\left(C_{1}\right)$ and $h\left(C_{2}\right)$. In short, $h(\partial D)$ is homotopically trivial in $M$. Hence there is a map $h^{\prime}: T \rightarrow M(\alpha)$ which agrees with $h$ on the complement of $D$, and maps $D$ into $M$. Then $h^{\prime}$ has lower complexity than $h$. This contradiction completes the proof that $A$ is essential and hence that $h$ satisfies (iv).

The following results are corollaries to Propositions 2.1 and 2.2.

Corollary 2.3. Let $M$ be a simple knot manifold, and let $F \subset M$ be an essential bounded surface. Let $\alpha$ be a slope in $\partial M$. If $M(\alpha)$ is very small, or more generally if $F \subset M \subset M(\alpha)$ is not $\pi_{1}$-injective in $M(\alpha)$, then there exists a singular surface $(S, X, h)$, well positioned with respect to $F$ and having boundary slope $\alpha$, such that $S$ is planar and $\partial S-X$ is non-empty and connected.

Proof. It follows from the definition of a simple manifold that if $F$ is a bounded essential surface in a simple knot manifold $M$, then $\chi(F)<0$, so that $\pi_{1}(F)$ is a non-abelian free group. Hence if $M(\alpha)$ is very small, then $F \subset M(\alpha)$ is not $\pi_{1^{-}}$ injective in $M(\alpha)$. We must show that if $F \subset M(\alpha)$ is not $\pi_{1}$-injective in $M(\alpha)$, then there exists a singular surface $(S, X, h)$ with the stated properties. We will apply Proposition 2.2, taking $T$ to be a disk and constructing the map $f: T \rightarrow M(\alpha)$ as follows. Since $F$ is not $\pi_{1}$-injective in $M(\alpha)$, there exists a homotopically non-trivial closed curve $\gamma$ on the surface $F$ which is null-homotopic in $M(\alpha)$. Deform the curve $\gamma$ by a homotopy to a curve $\gamma^{\prime}$ which is disjoint from $F$. Let $f$ be a null-homotopy of $\gamma^{\prime}$ in $M(\alpha)$. Then $f$ is $\pi_{1}$-injective since $\pi_{1}(T)$ is trivial, and condition (1) of Proposition 2.2 holds by construction. Since $F$ is essential, there does not exist a null-homotopy of $\gamma^{\prime}$ in $M$, so condition (2) of Proposition 2.2 holds as well.

Corollary 2.4. Let $M$ be a simple knot manifold, and let $F \subset M$ be an essential bounded surface. Let $\alpha$ be a slope in $\partial M$. If $M(\alpha)$ is reducible, then there exists a singular surface $(S, X, h)$, well positioned with respect to $F$ and having boundary slope $\alpha$, such that $S$ is planar and $X=\partial S$.

Proof. If $M(\alpha)$ is reducible, then there is an essential planar surface in $M$ with boundary slope $\alpha$. The assertion therefore follows from Proposition 2.1.

Corollary 2.5. Let $M$ be a simple knot manifold, and let $F \subset M$ be an essential bounded surface. Let $\alpha$ be a slope in $\partial M$ and let $g$ be a positive integer. Suppose that $\pi_{1}(M(\alpha))$ contains a subgroup which is isomorphic to the fundamental group of a closed connected surface of genus $g$, and that $\pi_{1}(M)$ does not. Then there exists 
a singular surface $(S, X, h)$, well positioned with respect to $F$ and having boundary slope $\alpha$, such that genus $S=g$ and $X=\partial S$.

Proof. If $\pi_{1}(M(\alpha))$ contains a genus- $g$ surface group, then there is a $\pi_{1}$-injective map from a surface of genus $g$ to $M(\alpha)$. Note that condition (1) of Proposition 2.2 holds vacuously, and condition (2) holds because of our hypothesis on $\pi_{1}(M(\alpha))$. The assertion therefore follows from Proposition 2.2.

Corollary 2.6. Let $M$ be a simple knot manifold, and let $F \subset M$ be an essential bounded surface. Let $\alpha$ be a slope in $\partial M$. If $M(\alpha)$ is a Seifert fibered space, then there exists a singular surface $(S, X, h)$, well positioned with respect to $F$ and having boundary slope $\alpha$, such that either (i) genus $S=1$ and $X=\partial S$, or (ii) $S$ is planar and $\partial S-X$ is non-empty and connected.

Proof. If $\pi_{1}(M(\alpha))$ is finite, or if $M(\alpha)$ is reducible and hence homeomorphic to $S^{1} \times S^{2}$ or $P^{3} \# P^{3}$, then $M(\alpha)$ is very small and conclusion (ii) holds by Corollary 2.3 .

If $\pi_{1}(M(\alpha))$ is infinite and $M(\alpha)$ is irreducible, then there exists a $\pi_{1}$-injective map $f: T \rightarrow M(\alpha)$, where $T$ is a torus. Since $M$ is atoroidal, any $\pi_{1}$-injective map from $T$ to $M$ is homotopic to a map into $\partial M$. But such a map cannot be $\pi_{1}$-injective as a map from $T$ to $M(\alpha) \supset M$, since $\partial M$ bounds a solid torus in $M(\alpha)$. It follows that $f$ is not homotopic to a map whose image is contained in $M$. Therefore $f$ satisfies the hypotheses of Proposition 2.2, which asserts the existence of a singular surface satisfying condition (i) of the statement.

\section{REDUCED HOMOTOPIES}

3.1. Basic homotopies. Let $M$ be a simple knot manifold and let $F$ be a transversely oriented essential surface in $M$. A homotopy in $(M, F)$ with domain $K$ is a homotopy $H$ with domain $K$ and target $M$ such that $H(K \times \partial I) \subset F$. A homotopy $H$ in $(M, F)$ is a basic homotopy if $H^{-1}(F)=K \times \partial I$.

We shall say that a basic homotopy $H:(K \times I, K \times \partial I) \rightarrow(M, F)$ starts on the + side (or the - side) if $H(K \times[0, \delta])$ is contained in $N_{+}(F)$ (or in $N_{-}(F)$ ) for sufficiently small $\delta>0$. Similarly, $H$ will be said to end on the + side (or the side) if $H(K \times[1-\delta, 1])$ is contained in $N_{+}$(or in $N_{-}$) for sufficiently small $\delta>0$. Of course in the case where $F$ separates $M$, a basic homotopy starts on the + side (or, respectively, the - side) if and only if it ends on the + side (or the - side). In this case we shall simply refer to it as a homotopy on the + side (or - side).

As a notational convention we will treat the symbols + and - as abbreviations for 1 and -1 , respectively. Thus if $\epsilon \in\{ \pm 1\}$ we may say that a homotopy $H$ starts on the $\epsilon$-side, meaning that it starts on the + side if $\epsilon=1$ or that it starts on the - side if $\epsilon=-1$.

There is a natural one-to-one correspondence between basic homotopies $H$ in $(M, F)$ with domain $K$ which start on the $\epsilon$ side, and homotopies $H^{\prime}$ in $\left(M_{F}, F_{-} \cup\right.$ $\left.F_{+}\right)$with domain $K$ such that $H^{\prime-1}\left(F_{-} \cup F_{+}\right)=K \times \partial I$ and $H_{0}^{\prime}(K) \subset F_{\epsilon}$. A basic homotopy $H$ is said to be essential if the corresponding homotopy

$$
H^{\prime}:(K \times I, K \times \partial I) \rightarrow\left(M, F_{-} \cup F_{+}\right)
$$

is an essential map of pairs. 


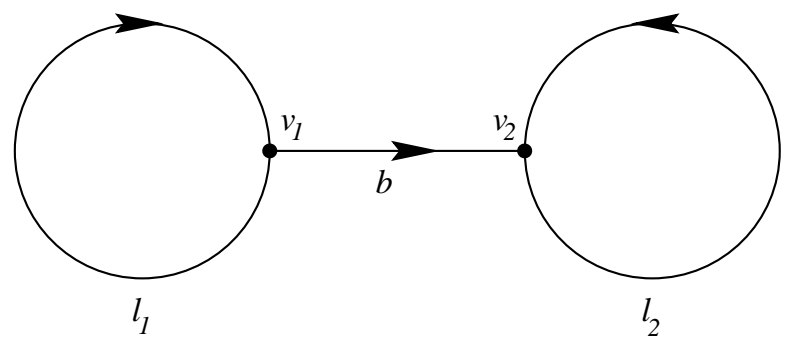

FIGURE 3.3.1

3.2. Reduced homotopies. Let $M$ be a simple knot manifold and $F$ an essential surface in $M$. Let $K$ be a finite polyhedron. Suppose that $n$ is a positive integer. A homotopy $H:(K \times I, K \times \partial I) \rightarrow(M, F)$ is said to be a reduced homotopy of length $n$ in $(M, F)$ if we may write $H$ as a composition of $n$ essential basic homotopies $H^{1}, \ldots, H^{n}$ in such a way that, for $i=1, \ldots n-1$, if $\epsilon_{i}$ denotes the element of $\{ \pm 1\}$ such that $H^{i+1}$ starts on the $\epsilon_{i}$ side, then we have that $H^{i}$ ends on the $-\epsilon_{i}$ side. If $\epsilon_{0}$ denotes the element of $\{ \pm 1\}$ such that $H^{1}$ starts on the $\epsilon_{0}$ side, then we shall also say that the reduced homotopy $H$ starts on the $\epsilon_{0}$ side.

We define a reduced homotopy of length 0 in $(M, F)$ to be a map $H$ from $K$ to $F$. The time-0 and time-1 maps of $H$ are defined to be $H$ itself. If $H$ is a reduced homotopy of length 0 and $H^{\prime}$ is a reduced homotopy of length $\geq 0$ whose time-1 (or time-0) map is equal to $H$, we define the composition of $H$ with $H^{\prime}$ (or of $H^{\prime}$ with $H$ ) to be $H^{\prime}$.

3.3. Let $\Gamma$ denote the 1-complex shown in Figure 3.3.1, consisting of two vertices $v_{1}$ and $v_{2}$, and three oriented edges $l_{1}, l_{2}$ and $b$. By an admissible pair of glasses in a surface $F$ we will mean a map $\gamma: \Gamma \rightarrow F$ such that the restriction of $\gamma$ to $\bar{b}$ is an essential path, and, for $i=1,2$, the restriction of $\gamma$ to $\bar{l}_{i}$ is a homeomorphism onto a boundary component of $F$. It is easy to show that if $\gamma$ is an admissible pair of glasses in a surface $F$ of negative Euler characteristic, then $\gamma:\left(\Gamma, \bar{l}_{1} \cup \bar{l}_{2}\right) \rightarrow(F, \partial F)$ is an essential map of pairs; and furthermore that if $\alpha$ is any map from $S^{1}$ to $\Gamma$ which is not homotopic into $l_{1}$ or $l_{2}$, then $g=\gamma \circ \alpha: S^{1} \rightarrow F$ is essential. In particular this remark applies when $F$ is an essential surface in a simple knot manifold, since the definition of simplicity then implies that $\chi(F)<0$.

3.4. Let $F$ be an essential surface in a simple knot manifold $M$. Suppose that $f:(I, \partial I) \rightarrow(F, \partial F)$ is an essential path and that

$$
H:(I \times I, I \times \partial I) \rightarrow(M, F)
$$

is a reduced homotopy of length $n$ such that $H_{0}=f$ and $H_{t}(\partial I) \subset \partial M$ for all $t \in I$. Let us identify the set $\bar{b} \subset \Gamma$ with $I$, respecting the orientations, so that $f$ becomes a map from $\bar{b}$ to $F$. Let us fix a product structure on $\partial M \cong S^{1} \times S^{1}$ such that each simple closed curve in $\partial F$ has the form $\{x\} \times S^{1}$ for some $x \in S^{1}$, and for $i=1,2$ let us fix orientation-respecting identifications of $\bar{l}_{i}$ with $S^{1}$. In this situation we shall describe a canonical way to extend $f$ to an admissible pair of glasses $\hat{f}: \Gamma \rightarrow F$, and to extend $H$ to a reduced homotopy $\hat{H}$ of length $n$ in the pair $(M, F)$ such that $\hat{H}_{0}=\hat{f}$. The reduced homotopy $\hat{H}$ will have the additional 
property that for all $t \in[0,1]$ the two closed curves $H_{t} \mid \bar{l}_{i}, i=1,2$, are homotopic to boundary components of $F$.

For $i=1,2$ the point $f\left(v_{i}\right)$ lies in a component $\left\{x_{i}\right\} \times S^{1}$ of $\partial F$. As we have identified $l_{i}$ with $S^{1}$, there is a unique rotation $\rho_{i}$ of $S^{1}$ such that $f\left(v_{i}\right)=\left(x_{i}, \rho_{i}\left(v_{i}\right)\right)$. We extend $f$ to a map $\hat{f}: \Gamma \rightarrow F$ by sending $\theta \in \bar{l}_{i}$ to $\left(x_{i}, \rho_{i}(\theta)\right)$ for $i=1,2$. Since the map $f$ is an essential path, it follows that $\hat{f}$ is an admissible pair of glasses. Similarly, we extend $H$ to a homotopy $\hat{H}$ as follows. For $i=1,2$ we map each $(\theta, t) \in \bar{l}_{i} \times I$ to $\left(x_{i, t}, \rho_{i, t}(\theta)\right)$, where $x_{i, t}$ is chosen so that $H\left(v_{i}, t\right) \in\left\{x_{i, t}\right\} \times S^{1}$, and $\rho_{i, t}$ is the unique rotation of $S^{1}$ such that $H\left(v_{i}, t\right)=\left(x_{i, t}, \rho_{i, t}\left(v_{i}\right)\right)$. Since $H$ is a reduced homotopy of length $n$, it is clear that $\hat{H}$ is also a reduced homotopy of length $n$.

3.5. Suppose that $F$ is an essential surface in a simple knot manifold $M$ and that $\gamma: \Gamma \rightarrow F$ is an admissible pair of glasses. Any length- $n$ reduced homotopy $\hat{H}$ in $(M, F)$ with domain $\Gamma$ and time- 0 map $\gamma$ can be used to produce a length- $n$ reduced homotopy in $(M, F)$ whose domain is $S^{1}$, and whose time-0 map is an essential map of $S^{1}$ into $F$. We map $S^{1}$ to the circuit in $\Gamma$ corresponding to the edge path $l_{1} b l_{2} b^{-1}$. Composing this map with $\gamma$ we obtain a map $g: S^{1} \rightarrow F$. The map $g$ is essential by the remark in Subsection 3.3. Composing $\hat{H}$ with $g \times$ id we obtain a reduced homotopy of length $n$ whose time- 0 map is $g$.

Definition 3.6. Let $G$ be a (possibly disconnected) graph in the interior of a compact surface $S$, and let $E$ and $E^{\prime}$ be distinct edges of $G$. We shall say that $E$ and $E^{\prime}$ are adjacent parallel edges if there exists a topological disk $D$, whose boundary is a union of two $\operatorname{arcs} A_{1}$ and $A_{2}$ with $A_{1} \cap A_{2}=\partial A_{1}=\partial A_{2}$, and a map $i: D \rightarrow S$, such that $i \mid\left(D-\partial A_{1}\right)$ is one-to-one, $i\left(A_{j}\right)=E_{j}$ for $j=1,2$, and $i$ (int $D) \cap G=\emptyset$. We shall say that $E$ and $E^{\prime}$ are parallel edges if there exist edges $E=E_{1}, \ldots, E_{n}=E^{\prime}$ such that $E_{i}$ and $E_{i+1}$ are adjacent parallel edges for $i=i, \ldots, n-1$. Note that parallelism is an equivalence relation on the set of edges of a graph.

3.7. For the statement of the next lemma we introduce the following notation, which will be used throughout the rest of the paper. If $s \geq 0, n \geq 0$ and $v>0$ are integers, we define

$$
N(s, n, v)=\max \left(1,6+\left[\frac{12 s+6 n-12}{v}\right]\right) .
$$

Lemma 3.8. Let $S$ be a compact, connected orientable surface having genus $s \geq 0$ and $n \geq 0$ boundary components. Let $G \subset$ int $S$ be a non-empty (but possibly disconnected) graph with $v>0$ vertices. Assume that no two (distinct) edges of $G$ are parallel, and that no loop in $G$ bounds a disk whose interior is disjoint from $G$. Then $G$ has a vertex of valence at most $N(s, n, v)$.

Proof. We may assume without loss of generality that $G$ has no vertex of valence 0 or 1.

Let $\phi$ be any component of $S-G$. Since $G$ has no valence-0 vertices, there exist a compact surface (with boundary) $\hat{\phi}$ and a map $i_{\phi}: \hat{\phi} \rightarrow S$, such that $i_{\phi}$ maps int $\hat{\phi}$ homeomorphically onto $\phi$, and $\partial \hat{\phi}$ has a cell decomposition in which every edge or vertex is mapped homeomorphically by $i_{\phi}$ onto an edge or vertex of $G$. We denote by $o(\phi)$ the number of edges in the cell decomposition of $\partial \hat{\phi}$. 
We claim that

$$
o(\phi) \geq 3 \chi(\hat{\phi})
$$

for every component $\phi$ of $S-G$. This is clear if $\bar{\phi}$ is not a disk, as in that case $\chi(\hat{\phi}) \leq 0$. Now suppose that $\bar{\phi}$ is a disk; we need to show that $o(\phi) \geq 3$. If $o(\phi)=1$, then there is a loop in $G$ bounding a disk whose interior is disjoint from $G$; this contradicts the hypothesis. If $o(\phi)=2$, and $i_{\phi}$ maps the edges of $\hat{\phi}$ to distinct edges $E_{1}$ and $E_{2}$ of $G$, then $E_{1}$ and $E_{2}$ are parallel, and again the hypothesis is contradicted. If $o(\phi)=2$, and $i_{\phi}$ maps the edges of $\hat{\phi}$ to the same edge of $G$, then $S \cong S^{2}$ and the graph $G$ has one edge and two vertices. This contradicts our assumption that $G$ has no vertices of valence 1 . Thus $(*)$ is proved in all cases.

Summing (*) over the components of $S-G$, we find that

$$
2 e=\sum_{\phi} o(\phi) \geq 3 t
$$

where $e$ denotes the number of edges of $G$ and

$$
t=\sum_{\phi} \chi(\hat{\phi})
$$

Now

$$
2-2 s-n=\chi(S)=v-e+t \leq v-\frac{e}{3} .
$$

If $k$ denotes the minimum valence of any vertex of $G$, then we have $2 e \geq k v$, and so

$$
2-2 s-n \leq v-\frac{k v}{6} .
$$

Since $k$ is an integer it follows that $k \leq N(s, n, v)$.

Proposition 3.9. Let $M$ be a simple knot manifold. Let $F$ be a bounded connected essential surface in $M$ with boundary slope $\beta$ and $m$ boundary components. Let $(S, X, h)$ be a singular surface in $M$ which has boundary slope $\alpha \neq \beta$ and is well positioned with respect to $F$. Set $s=$ genus $S, n=|\partial S-X|, v=|X|$.

Then there exists an essential homotopy $H: I \times I \rightarrow M$ having length at least

$$
\frac{m \Delta(\alpha, \beta)}{N(s, n, v)}-1
$$

such that $H_{0}$ is an essential path in $F$ and $H_{t}(\partial I) \subset \partial M$ for all $t \in I$.

Proof. The definition of a singular surface gives $X \neq \emptyset$, so that $v>0$. Let $\hat{S}$ denote the surface obtained from $S$ by identifying each component of $X$ to a point. The surface $\hat{S}$ contains a non-empty graph whose vertices are the $v$ points in the image of $X$, and whose edges are the images of the arc components of $h^{-1}(F)$. Each component of $h(X)$ is a curve on $\partial M$ of slope $\alpha$, and each component of $\partial F$ is a curve of slope $\beta$. Thus it follows from standard position that each component of $X$ meets $h^{-1}(F)$ in $m \Delta(\alpha, \beta)$ points. In other words, each vertex of the graph $G$ has valence $m \Delta(\alpha, \beta)$, which is strictly positive since $\alpha \neq \beta$. Since $(S, X, h)$ is well positioned with respect to $F$, there is no loop in $G$ bounding a disk whose interior is disjoint from $G$.

We may apply Lemma 3.8 to a subgraph of $G$ containing exactly one edge from each class of parallel edges. We conclude that the edges emanating from some vertex of $G$ fall into at most $N(s, n, v)$ parallel classes. Thus there must exist some class 
of parallel edges containing at least $m \Delta(\alpha, \beta) / N(s, n, v)$ edges. Label the edges of this class $E_{1}, \ldots, E_{k}$, where $k \geq m \Delta(\alpha, \beta) / N(s, n, v)$. For each $i=1, \ldots, k$ let $A_{i}$ be the arc component of $h^{-1}(F)$ which maps to $E_{i}$ under the quotient map from $S$ to $\hat{S}$. For each $i=1, \ldots, k-1$ there is a disk $Q_{i}$ in $S$ bounded by two subarcs of $X$ together with $A_{i}$ and $A_{i+1}$. Because $(S, X, h)$ is well positioned with respect to $F$, the interior of $Q_{i}$ is disjoint from $f^{-1}(F)$. The restriction of $h$ to this quadrilateral disk defines a basic homotopy $H^{i}$ whose time- 0 map is the path $h\left(A_{i}\right)$ and whose time- 1 map is the path $h\left(A_{i+1}\right)$. The paths $h\left(A_{i}\right)$ are essential because $(S, X, h)$ is well positioned with respect to $F$. It is an immediate consequence of standard position that each of the basic homotopies $H^{i}$ is essential; since $h$ is transverse to $F$, the composition of $H^{1} \ldots H^{k-1}$ is a reduced homotopy of length $k-1$. The time-0 map of this reduced homotopy is the essential path $h\left(A_{1}\right)$. Since $k \geq m \Delta(\alpha, \beta) / N(s, n, v)$, this completes the proof of the proposition.

Corollary 3.10. Let $M$ be a simple knot manifold that contains an essential surface $F$ with boundary slope $\beta$. Suppose that the pair $\left(M_{F}, F_{-} \cup F_{+}\right)$is acylindrical. Let $(S, X, h)$ be a singular surface with boundary slope $\alpha$ which is well positioned with respect to $F$. Set $s=$ genus $S, n=|\partial S-X|, v=|X|$, and $m=|\partial F|$. Then

$$
\Delta(\alpha, \beta) \leq \frac{N(s, n, v)}{m} .
$$

Proof. We may assume $\alpha \neq \beta$. According to Proposition 3.9, there exist an essential path $\gamma: I \rightarrow F$ and a reduced homotopy in $(M, F)$ which has time-0 map $\gamma$ and length at least

$$
l \geq \frac{m \Delta(\alpha, \beta)}{N(s, n, v)}-1
$$

It therefore follows from Subsections 3.4 and 3.5 that there exists a reduced homotopy of length $l$ in $(M, F)$ whose time-0 map is a (possibly singular) essential curve in $M$. But since $\left(M_{F}, F_{-} \cup F_{+}\right)$contains no properly embedded essential annuli, the Annulus Theorem implies that there can exist no essential basic homotopy in $M$ whose time-0 map is an essential curve in $F$. Hence we must have

$$
\frac{m \Delta(\alpha, \beta)}{N(s, n, v)}-1 \leq 0
$$

which is equivalent to the conclusion of the corollary.

Corollary 3.11. Let $M$ be a simple knot manifold that contains an essential surface $F$ with boundary slope $\beta$. Suppose that the pair $\left(M_{F}, F_{-} \cup F_{+}\right)$is acylindrical. Let $\alpha$ be a slope on $\partial M$.

(1) If $M(\alpha)$ is a very small manifold, then $\Delta(\alpha, \beta) \leq 5$.

(2) If $M(\alpha)$ is a Seifert fibered space, then $\Delta(\alpha, \beta) \leq 6$.

Proof. To prove (1) we invoke Corollary 2.3 to obtain a singular surface $(S, X, h)$, well positioned with respect to $F$, such that genus $S=0$ and $|\partial S-X|=1$. The conclusion now follows from Corollary 3.10 because for any $v \geq 1$ we have $N(0,1, v) \leq 5$. To prove (2) we invoke Corollary 2.6 to obtain a singular surface $(S, X, h)$, well positioned with respect to $F$, such that either genus $S=0$ and $|\partial S-X|=1$, or genus $S=1$ and $|\partial S-X|=0$. The conclusion now follows from Corollary 3.10 because for any $v \geq 1$ we have $N(0,1, v) \leq 5$ and $N(1,0, v)=6$. 


\section{Essential InTERSECtions}

This section introduces a version of the notion of essential intersection for subsurfaces of a 2-manifold, a notion which has appeared implicitly in much of the literature on the characteristic submanifold of a Haken manifold. The version we present here is adapted to the case of "large" subsurfaces, which we now define.

Let $S$ be a compact orientable surface. We say that a subsurface $A$ of $S$ is large if each component of $A$ is $\pi_{1}$-injective and has negative Euler characteristic. Note that the empty set is considered to be a large subsurface according to this definition. If the components of $S$ have Euler characteristic $\geq 0$, then the empty set is the only large subsurface.

The large part of a $\pi_{1}$-injective subsurface $A$ of $S$, denoted by $\mathcal{L}(A)$, is the union of all the large components of $A$.

The next lemma is preliminary to the proof of Proposition 4.2, which will provide the definition of the "large intersection" of two large subsurfaces.

Lemma 4.1. Let $A$ and $B$ be large subsurfaces of a compact orientable surface $S$, and suppose that $A$ is homotopic into $B$.

(1) $A$ is isotopic in $S$ to a subsurface of $B$.

(2) If $B$ is homeomorphic to a large subsurface of $A$, then $A$ and $B$ are isotopic subsurfaces of $S$.

(3) If $B$ is homotopic into $A$, then $A$ and $B$ are isotopic subsurfaces of $S$.

Proof. Without loss of generality we may assume that $S$ is connected.

We begin with the proof of (1). We may assume that $A$ has been chosen within its isotopy class so that $\partial A$ meets $\partial B$ transversely and in the minimal number of points. Moreover, we may assume that $A$ has been chosen, among all surfaces in its isotopy class which minimize $|\partial A \cap \partial B|$, to minimize the number components of $\partial A$ which are not contained in $B$. Under these assumptions we will show that $A \subset B$, proving (1).

Let $A_{0}$ be any component of $A$. By hypothesis, $A_{0}$ is homotopic into a component $B_{0}$ of $B$. We will prove that $A_{0} \subset B_{0}$. As $A_{0}$ is an arbitrary component of $A$, the assertion will follow.

Fix a base point in $B_{0}$, and consider the covering $p: \tilde{S} \rightarrow S$ determined by $i m\left(\pi_{1}\left(B_{0}\right) \rightarrow \pi_{1}(S)\right)$. There is a subsurface $\tilde{B}_{0}$ of $\tilde{S}$ which is mapped homeomorphically onto $B_{0}$ by $p$. Since $B_{0}$ is large, $\partial B_{0}$ is $\pi_{1}$-injective in $S$, and hence each component of $X=\tilde{S}-i n t \tilde{B}_{0}$ is a half-open annulus. Since $A_{0}$ is homotopic into $B_{0}$, the inclusion $i: A_{0} \rightarrow S$ is homotopic to a map which admits a lift to $\tilde{S}$. Hence $i$ itself admits a lift to $\tilde{S}$; that is, we have a subsurface $\tilde{A}_{0}$ of $\tilde{S}$ which is mapped homeomorphically onto $A_{0}$ by $p$. In order to show that $A_{0} \subset B_{0}$, it suffices to show that $\tilde{A}_{0} \subset \tilde{B}_{0}$.

As a preliminary, we will prove that $\partial \tilde{A}_{0}$ is disjoint from $\partial \tilde{B}_{0}$. Assume this is false. Then $\partial \tilde{A}_{0}$ contains a properly embedded arc $\tilde{\alpha} \subset X$. Since the component $X_{0}$ of $X$ containing $\tilde{\alpha}$ is a half-open annulus, $\partial \tilde{\alpha}$ is the boundary of an arc $\tilde{\beta} \subset$ $\partial X_{0} \subset \tilde{B}_{0}$, and $\tilde{\alpha} \cup \tilde{\beta}$ bounds a disk $\tilde{D} \subset \tilde{S}$. Among all disks in $\tilde{S}$ whose boundaries are made up of an arc in $p^{-1}(\partial A)$ and an arc in $p^{-1}(\partial B)$, choose one, say $\tilde{D}_{0}$, which is minimal with respect to inclusion. Write $\partial \tilde{D}_{0}=\tilde{\alpha}_{0} \cup \tilde{\beta}_{0}$, where $\tilde{\alpha}_{0} \subset p^{-1}(\partial A)$ and $\tilde{\beta}_{0} \subset p^{-1}(\partial B)$ are arcs with $\partial \tilde{\alpha}_{0}=\partial \tilde{\beta}_{0}$. We claim that $p \mid \partial \tilde{D}_{0}$ is one-to-one. It is clear from the minimality of $\tilde{D}_{0}$ that $p\left(\right.$ int $\left.\tilde{\alpha}_{0}\right) \cap p\left(\right.$ int $\left.\tilde{\beta}_{0}\right)=\emptyset$. If $p \mid$ int $\tilde{\alpha}_{0}$ is not one-to-one, then $\alpha=p\left(\right.$ int $\left.\tilde{\alpha}_{0}\right)$ is an entire component of $\partial A$, which contains 
$p\left(\partial \tilde{\alpha}_{0}\right)$ and therefore meets $B$; hence int $\tilde{\alpha}_{0}$ meets $p^{-1}(B)$, and the minimality of $\tilde{D}_{0}$ is contradicted. Thus $p \mid$ int $\tilde{\alpha}_{0}$ is one-to-one, and similarly $p \mid$ int $\tilde{\beta}_{0}$ is oneto-one. Hence if $p \mid \partial \tilde{D}_{0}$ is not one-to-one, then $\alpha_{0}=p\left(\tilde{\alpha}_{0}\right)$ and $\beta_{0}=p\left(\tilde{\beta}_{0}\right)$ are simple closed curves meeting in a single point; as these curves must be components, the intersection is transverse. But this is impossible, as the hypothesis that $A$ is homotopic into $B$ implies that $\alpha_{0}$ and $\beta_{0}$ are homotopic to disjoint curves. Thus $p \mid \partial D_{0}$ must be one-to-one.

According to [E Lemma 1.6], it follows that $p \mid \tilde{D}_{0}$ is one-to-one; hence $D_{0}=$ $p\left(\tilde{D}_{0}\right)$ is a disk in $S$ whose boundary consists of two $\operatorname{arcs}$, one in $B$ and one in $A$, and int $D_{0}$ is disjoint from $\partial A$ and from $\partial B$. It follows that $\partial B$ is isotopic to a curve system that meets $\partial A$ transversely in fewer points than $\partial B$, a contradiction to our choice of $B$. This proves that $\partial \tilde{A}_{0} \cap \partial \tilde{B}_{0}=\emptyset$.

We are now ready to prove that $\tilde{A}_{0} \subset \tilde{B}_{0}$. If this is false, then either $\tilde{A}_{0} \subset X$ or $\tilde{A}_{0} \cap \partial X \neq \emptyset$. If $\tilde{A}_{0} \subset X$, then since the components of $X$ are half-open annuli, $i m\left(\pi_{1}\left(A_{0}\right) \rightarrow \pi_{1}(S)\right)$ is cyclic, a contradiction since $A$ is a large subsurface of $S$. There remains the possibility that $\tilde{A}_{0}$ meets the boundary of some component $X_{1}$ of $X$. Since $\partial \tilde{A}_{0} \cap \partial \tilde{B}_{0}=\emptyset$, the compact subsurface $\tilde{Z}=\tilde{A}_{0} \cap X_{1}$ must have $\tilde{\gamma}_{1}=\partial X_{1}$ as one boundary component. If $\tilde{\gamma}$ is any other component of $\partial \tilde{Z}$, then $\tilde{\gamma}$ lies in the interior of the half-open annulus $X_{1}$; furthermore, $\tilde{\gamma}$ is a component of $\partial \tilde{A}_{0}$, and is therefore homotopically non-trivial (since the large subsurface $A_{0}$ of $S$ is in particular $\pi_{1}$-injective). It follows that $\tilde{Z}$ is an annulus bounded by $\tilde{\gamma}_{1}$ and a single component $\tilde{\gamma}_{2}$ of $\partial \tilde{A}_{0}$. Since $p$ maps $\tilde{A}_{0}$ homeomorphically onto $A_{0}$, it in particular maps $\tilde{Z}$ homemorphically onto an annulus $Z \subset A_{0}$. There is an isotopy supported on a small neighborhood $W$ of $Z$ in $S$ which carries $\gamma_{1}=p\left(\gamma_{1}\right)$ into int $B_{0}$. Since $W$ may be taken to meet $\partial A$ only in $\gamma_{1}$, it is clear that the image of $A$ under the isotopy has the same intersection with $B$ as $A$ has, and has fewer components $\partial A$ which are not contained in $B$ than $A$ has. This contradiction to the minimality of $A$ completes the proof of (1).

We now turn to the proof of (2). By part (1) we may assume that $A$ is a subsurface of int $B$. Let $B^{\prime}$ be a $\pi_{1}$-injective subsurface of int $A$ which is homeomorphic to $B$. Any disk component of $\overline{B-A}$ would also be a component of $\overline{S-A}$. Since $A$ is large it follows that no component of $\overline{B-A}$ is a disk. Hence $\chi(B) \leq \chi(A)$. Similarly, $\chi(A) \leq \chi\left(B^{\prime}\right)=\chi(B)$. Thus each component of $\overline{B-A}$ is an annulus, and each component of $\overline{A-B^{\prime}}$ is an annulus. Since $A$ and $B$ are large it follows that each component of $B$ contains at least one component of $A$ and that each component of $A$ contains at least one component of $B^{\prime}$. Since $B$ is homeomorphic to $B^{\prime}$, a given component of $B$ can contain only one component of $B^{\prime}$. Hence each component of $B$ contains exactly one component of $A$.

We may therefore index the components of $A$ as $A_{1}, \ldots, A_{n}$, and index the components of $B$ as $B_{1}, \ldots, B_{n}$, in such a way that $A_{i}$ is a subsurface of $B_{i}$ for $i=1, \ldots, n$, and each component of $\overline{B_{i}-A_{i}}$ is an annulus.

To complete the proof of (2) it suffices to show that there is no annulus component of $\overline{B_{i}-A_{i}}$ whose boundary is contained in $A_{i}$. If such an annulus component did exist the genus of $B_{i}$ would be strictly greater than the genus of $A_{i}$. In particular we would have genus $B=\sum$ genus $B_{i}>\sum$ genus $A_{i}=$ genus $A$. (Recall that genus $B$ denotes the total genus of $B$.) But since $B$ is homeomorphic to the subsurface $B^{\prime}$ of $A$, we have genus $B=$ genus $B^{\prime} \leq$ genus $A$. This contradiction completes the proof of (2). 
To prove (3), note that if $B$ is homotopic into $A$, then by (1) it is isotopic into $A$. The time-1 map of the isotopy is a homeomorphism onto a subsurface of $A$ which is clearly large since it is isotopic to $B$. It now follows from (2) that $A$ and $B$ are isotopic.

A map $f$ from a finite polyhedron $K$ to a surface $S$ is called large if for each component $K_{0}$ of $K$, the subgroup $f_{\#}\left(\pi_{1}\left(K_{0}\right)\right)$ of $\pi_{1}(S)$ is non-abelian.

Proposition 4.2. Suppose that $A$ and $B$ are large subsurfaces of a compact orientable surface $S$. Then up to non-ambient isotopy there is a unique large subsurface $C$ of $S$ with the following property:

(*) Any large map from a polyhedron into $S$ is homotopic into $C$ if and only if it is homotopic into $A$ and homotopic into $B$.

Furthermore, if $C \subset$ int $S$ is a large subsurface satisfying $(*)$, then there are subsurfaces $A_{0} \subset$ int $S$ and $B_{0} \subset$ int $S$, isotopic to $A$ and $B$, such that $\partial A_{0}$ and $\partial B_{0}$ meet transversely and $C=\mathcal{L}\left(A_{0} \cap B_{0}\right)$.

Proof. We may assume without loss of generality that $A, B \subset$ int $S$. We prove uniqueness first. Suppose that $C$ and $C^{\prime}$ are large subsurfaces of $S$ satisfying (*). Then the inclusion $C \rightarrow S$ is homotopic into $C^{\prime}$, and vice versa. Lemma 4.1(3) now implies that $C$ and $C^{\prime}$ are isotopic in $S$.

To complete the proof of the proposition, it suffices to show that there is a subsurface $B_{0}$ of int $S$ isotopic to $B$, such that $\partial A$ and $\partial B_{0}$ meet transversely and such that $\mathcal{L}\left(A \cap B_{0}\right)$ satisfies $(*)$. We define $B_{0}$ as follows: among all subsurfaces of int $S$ which are isotopic to $B$, and whose boundaries meet $\partial A$ transversely, we choose $B_{0}$ so that the number of points of $\partial A \cap \partial B_{0}$ is as small as possible. We set $C=\mathcal{L}\left(A \cap B_{0}\right)$.

It is clear that if a large map from a connected polyhedron $K$ into $S$ is homotopic into $C$, then it is homotopic into $A$ and homotopic into $B$. The proof of $(*)$ will be completed by showing that, conversely, if a large map $f: K \rightarrow S$ is homotopic into $A$ and homotopic into $B$, then it is homotopic into $C$. We may assume without loss of generality that $K$ is non-empty and connected. We fix a component $A_{1}$ of $A$ such that $f$ is homotopic into $A_{1}$.

Let $\tilde{S}$ denote the covering space of int $S$ corresponding to the subgroup

$$
i m\left(\pi_{1}\left(A_{1}\right) \rightarrow \pi_{1}(\text { int } S)\right) \text {. }
$$

Then $\tilde{S}$ has a subsurface $\tilde{A}$ such that the covering projection $p: \tilde{S} \rightarrow$ int $S$ maps $\tilde{A}$ homeomorphically onto $A_{1}$. Set $X=\tilde{S}-i n t \tilde{A}$. Since $A$ is $\pi_{1}$-injective, each component of $X$ is a half-open annulus meeting $\tilde{A}$ in a component of $\partial \tilde{A}$.

Since $f: K \rightarrow S$ is homotopic into $A_{1}$ it admits a lift $\tilde{f}$ to the covering space $\tilde{S}$. Since $f$ is also homotopic into $B_{0}$, there is a homotopy from $\tilde{f}$ to a map $g: K \rightarrow \tilde{S}$ such that $g(K)$ is contained in a component $\tilde{B}_{0}$ of $p^{-1}\left(B_{0}\right)$.

We claim that $\tilde{B}_{0} \cap \tilde{A} \neq \emptyset$. Suppose not; then $g(K) \subset \tilde{B}_{0} \subset X$. But $g_{\sharp}\left(\pi_{1}(K)\right) \subset$ $\pi_{1}(\tilde{S})$ is non-abelian since $f$ is a large map. Since each component of $X$ is a halfopen annulus, we have a contradiction, and the claim is proved.

We next claim that if $Z$ is any component of $\tilde{B}_{0} \cap X$, then $Z$ deforms into $W=Z \cap \partial X \subset \partial \tilde{A}$. (This means that the identity map of $Z$ is homotopic in $Z$, rel $W$, to a map whose image is contained in $W$.) To prove the claim, first note that $W$ is a 1-manifold in the boundary of the 2-manifold $Z$. Furthermore, since $\tilde{B}_{0} \cap \tilde{A} \neq \emptyset$, we have $W \neq \emptyset$. Hence, in order to prove that $Z$ deforms into $W$, 
it suffices to show that if $\alpha \subset Z$ is an arc with endpoints in $W$, then $\alpha$ is parallel in $Z$ to an $\operatorname{arc}$ in $W$. Since $X$ is a half-open annulus, $\alpha$ is parallel in $X$ to an arc $\beta \subset \partial X$. We must show that the disk $D \subset X$ bounded by $\alpha \cup \beta$ is contained in $Z$.

If $D \not \subset Z$, then $D$ contains a component $\gamma$ of the frontier of $Z$ relative to $X$. Note that $\gamma \subset \partial \tilde{B}_{0}$, and that $\gamma$ is a properly embedded 1-manifold in $X$. But $\gamma$ cannot be a simple closed curve, since $B_{0}$ is $\pi_{1}$-injective in $S$; hence $\gamma$ is an arc whose endpoints lie in $\beta$. Thus there is a disk in $\tilde{S}$ whose boundary is the union of the $\operatorname{arc} \gamma \subset p^{-1}\left(\partial B_{0}\right)$ and a sub-arc of $\beta \subset p^{1}(\partial A)$. Among all disks in $\tilde{S}$ bounded by the union of an arc in $p^{-1}(\partial A)$ with an arc in $p^{-1}\left(\partial B_{0}\right)$, choose one, say $D^{\prime}$, which is minimal with respect to inclusion. According to [E, Lemma 1.6], $p \mid D^{\prime}$ is one-to-one and hence $p\left(D^{\prime}\right)$ is a disk in $S$ whose boundary consists of two arcs, one in $B_{0}$ and one in $A$, which meet at their endpoints. Since $B_{0}$ is large we have int $p\left(D^{\prime}\right) \cap B_{0}=\emptyset$. We may therefore isotope $B_{0}$ across the disk $p\left(D^{\prime}\right)$ to obtain a surface whose boundary meets $\partial A$ in fewer points than $\partial B_{0}$. This contradicts our choice of $B_{0}$, and the claim is proved.

We have shown that $\tilde{B}_{0} \cap \tilde{A} \neq \emptyset$ and that every component of $\tilde{B}_{0} \cap X$ deforms into its intersection with $\partial X=\partial \tilde{A}$. It follows that $\tilde{B}_{0}$ deforms into $\tilde{B}_{0} \cap \tilde{A}$. Since $\tilde{f}$ is homotopic to $g$ and $g(K) \subset \tilde{B}_{0}$, it follows that $\tilde{f}$ is homotopic in $\tilde{S}$ to a map of $K$ into $\tilde{B}_{0} \cap \tilde{A}$. In particular, $f$ is homotopic in $S$ to a map $f^{\prime}$ whose image is contained in $B_{0} \cap A_{1} \subset B_{0} \cap A$. As $f$ is large it follows that $f(K) \subset \mathcal{L}\left(B_{0} \cap A\right)=C$.

Definition 4.3. If $A$ and $B$ are large subsurfaces of a compact orientable surface $S$, then the subsurface provided by Proposition 4.2 , which is well defined up to non-ambient isotopy, will be called the large intersection of $A$ and $B$ and will be denoted $A \wedge_{\mathcal{L}} B$. Clearly $A \wedge_{\mathcal{L}} B$ is isotopic to $B \wedge_{\mathcal{L}} A$, and $A \wedge_{\mathcal{L}}\left(B \wedge_{\mathcal{L}} C\right)$ is isotopic to $\left(A \wedge_{\mathcal{L}} B\right) \wedge_{\mathcal{L}} C$.

The following result will be needed in the next two sections.

Proposition 4.4. Suppose that $A$ is a large subsurface of a compact orientable surface $S$, and that $f$ and $g$ are large maps of a polyhedron $K$ into A. If $f$ and $g$ are homotopic in $S$, then they are homotopic in $A$.

Proof. We may assume that $K$ and $S$ are connected. Let $A_{0}$ denote the component of $A$ containing $f(K)$, and let $p: \tilde{S} \rightarrow S$ denote the largest covering space of $S$ to which the inclusion map $i: A_{0} \rightarrow S$ lifts. If $\tilde{i}: A_{0} \rightarrow \tilde{S}$ is the lift of $i$, then $\tilde{A}_{0}=\tilde{i}\left(A_{0}\right)$ is a deformation retract of $\tilde{S}$, and each component of $\tilde{S}-\tilde{A}_{0}$ has cyclic (and possibly trivial) fundamental group. Let $\tilde{f}: K \rightarrow \tilde{S}$ be a lift of $f$ with $\tilde{f}(K) \subset \tilde{A}_{0}$, and let $\tilde{g}: K \rightarrow \tilde{S}$ be a lift of $g$ which is homotopic to $\tilde{f}$ in $\tilde{S}$. Since $g$ is large, $\tilde{g}\left(\pi_{1}(K)\right)$ is not cyclic; thus $\tilde{g}(K)$ cannot be contained in a component $\tilde{A}_{0}^{\prime} \neq \tilde{A}_{0}$ of $p^{-1}\left(A_{0}\right)$. Hence $\tilde{g}(K) \subset \tilde{A}_{0}$, and $\tilde{f}$ and $\tilde{g}$ are homotopic in $\tilde{A}_{0}$. The conclusion follows.

Lemma 4.5. Let $A$ be a large subsurface of a compact orientable surface $B$, and suppose that $B$ admits an involution $\tau$ such that $\tau(A)$ is isotopic to $A$ in $B$. Then $A$ is isotopic to a subsurface $A^{\prime}$ of $B$ such that $\tau\left(A^{\prime}\right)=A^{\prime}$.

Proof. Choose a negatively curved metric on $B$ which is invariant under the involution $\tau$ and has the property that the boundary components of $B$ are geodesics. Given a large subsurface $C$ of $B$ we divide its boundary components into two types:

Type 1: those which are not isotopic to any other boundary component of $C$. 
Type 2: those which are isotopic to some other boundary component of $C$.

There is a small positive real number $t_{0}$ such that $A$ can be isotoped in $B$ to a surface $A^{\prime}$ whose type 1 boundary components are geodesics and whose type 2 boundary components form the boundary of a $t_{0}$-bicollar of a finite union of geodesics. Then $\partial A^{\prime}$ is invariant under the involution $\tau$. To see that $\tau\left(A^{\prime}\right)=A^{\prime}$ let $A_{0}^{\prime}$ be a component of $A^{\prime}$. If $\tau\left(A_{0}^{\prime}\right) \not \subset A^{\prime}$, then the interior of $\tau\left(A_{0}^{\prime}\right)$ is disjoint from $A^{\prime}$ and hence we may isotope $\tau\left(A_{0}^{\prime}\right)$ into the complement of $A^{\prime}$. Let $p: \tilde{B} \rightarrow B$ be the maximal connected cover for which the inclusion map $i: \tau\left(A_{0}^{\prime}\right) \rightarrow B$ lifts to a map $\tilde{i}: \tau\left(A_{0}^{\prime}\right) \rightarrow \tilde{B}$ and define $Y=\tilde{i}\left(\tau\left(A_{0}^{\prime}\right)\right)$. Then $X=\tilde{B}-\operatorname{int} Y$ is a disjoint union of half-open annuli. By hypothesis $\tilde{i}$ is homotopic to a map with image in $X$ so that in particular $Y$ may be homotoped into an annulus. It follows from Lemma 4.1 that $Y$ is isotopic to a subsurface of an annulus. Since $Y$ is $\pi_{1}$-injective and homeomorphic to $A_{0}$, this contradicts our assumption that $A_{0}$ is large. Thus $\tau\left(A_{0}^{\prime}\right) \subset A^{\prime}$ and we deduce that $\tau\left(A^{\prime}\right)=A^{\prime}$.

4.6. Let $A$ be a subsurface of a compact orientable surface $S$. By an outer component of $A$ we will mean a component of $A$ which contains a simple closed curve that is isotopic to a boundary component of $S$. We will say that $A$ is outer if every component of $A$ is outer. We define the outer part of $A$, denoted $\dot{A}$, to be the union of all outer components of $A$, i.e. the largest outer subsurface of $A$. Thus $A$ is an outer subsurface if and only if $A=\dot{A}$.

If $A$ and $B$ are subsurfaces of $S$ with $A \subset B$, note that $\dot{A} \subset \dot{B}$. If $A$ and $B$ are large subsurfaces of $S$, we define $A \dot{\wedge}_{\mathcal{L}} B$ to be the outer part of $A \wedge_{\mathcal{L}} B$. It is a formal consequence of this definition that a large outer subsurface of $S$ is homotopic into both $A$ and $B$ if and only if it is homotopic into $A \dot{\wedge}_{\mathcal{L}} B$. It follows from this, together with Lemma 4.1, that if $A, B$ and $C$ are large subsurfaces of $S$, then $A \dot{\wedge}_{\mathcal{L}} B$ is homotopic to $B \dot{\wedge}_{\mathcal{L}} A$ and $\left(A \dot{\wedge}_{\mathcal{L}} B\right) \dot{\wedge}_{\mathcal{L}} C$ is isotopic to $A \dot{\wedge}_{\mathcal{L}}\left(B \dot{\wedge}_{\mathcal{L}} C\right)$.

Lemma 4.7. Suppose that $A$ and $B$ are large subsurfaces of a compact orientable surface $S$. Then $A \dot{\wedge}_{\mathcal{L}} B$ is isotopic in $S$ to $\dot{A} \dot{\wedge}_{\mathcal{L}} \dot{B}$.

Proof. Since each component of $\dot{A} \dot{\wedge}_{\mathcal{L}} \dot{B}$ contains a simple closed curve that is isotopic to a boundary component of $S$, it is clear that $A \dot{\wedge}_{\mathcal{L}} B \supset \dot{A} \dot{\wedge}_{\mathcal{L}} \dot{B}$. To prove the reverse inclusion, let $C$ be a component of $A \dot{\wedge}_{\mathcal{L}} B$. We have that $C$ is isotopic into $X \wedge_{\mathcal{L}} Y$, where $X$ and $Y$ are components of $A$ and $B$ respectively. Since $C$ contains a simple closed curve isotopic to a boundary component of $S$, so do $X$ and $Y$. Thus $X$ and $Y$ are in fact components of $\dot{A}$ and $\dot{B}$, respectively. This shows that $C$ is isotopic into a component of $\dot{A} \wedge_{\mathcal{L}} \dot{B}$. But, since $C$ contains a simple closed curve isotopic to a boundary component of $S$, it is isotopic into a component of $\dot{A} \dot{\wedge}_{\mathcal{L}} \dot{B}$. Since $C$ was an arbitrary component of $A \dot{\wedge}_{\mathcal{L}} B$, we have shown that $A \dot{\wedge}_{\mathcal{L}} B$ is homotopic into $\dot{A} \dot{\Lambda}_{\mathcal{L}} \dot{B}$. The lemma now follows from Lemma 4.1.

\section{Reduced Homotopies AND THE CHARACTERISTIC PAIR}

The final result of this section, Theorem 5.4.1, concerns a pair $(M, F)$, where $M$ is a simple knot manifold and $F \subset M$ is an essential surface in $M$ which is not semi-fiber. The theorem provides an upper bound, in terms of the genus and the number of boundary components of $F$, for the length of a reduced homotopy in $(M, F)$ having a large time-0 map. 


\subsection{Splitting surfaces.}

Definition 5.1.1. Let $M$ be simple knot manifold. A splitting surface in $M$ is a transversely oriented essential surface $\tilde{F} \subset M$ such that $M_{\tilde{F}}$ is a disjoint union of two compact submanifolds $M_{\tilde{F}}^{+}$and $M_{\tilde{F}}^{-}$with the property that $N_{\epsilon}(\tilde{F}) \subset M_{\tilde{F}}^{\epsilon}$ for $\epsilon= \pm 1$.

It is easy to see that $M_{\tilde{F}}^{+}$and $M_{\tilde{F}}^{-}$are uniquely determined by $\tilde{F}$. Note that any transversely oriented, separating, connected, essential surface in $M$ is a splitting surface. In general, however, a splitting surface need not be connected.

Since $\tilde{F}$ comes equipped with a transverse orientation, any orientation of $M$ induces an orientation of $\tilde{F}$. An orientation of $\tilde{F}$ will be called consistent if it is induced from an orientation of $M$ in this way. If $\tilde{F}$ has $n$ components, then it has $2^{n}$ possible orientations; but since $M$ is connected, only two of these orientations are consistent.

If $\tilde{F}$ is a splitting surface, then for $\epsilon= \pm 1$ the natural map from $M_{\tilde{F}}^{\epsilon}$ to $M$ is injective and we shall identify $M_{\tilde{F}}^{+}$and $M_{\tilde{F}}^{-}$with submanifolds of $M$. In particular $\tilde{F}_{\epsilon}$ is identified with $\tilde{F}$ via the homeomorphism $i_{\epsilon}$ (see Subsection 1.3). Furthermore we shall identify basic homotopies in $(M, F)$ with the corresponding homotopies in $\left(M_{\tilde{F}}, \tilde{F}_{-} \cup \tilde{F}_{+}\right)($see Subsection 3.1$)$.

An orientation of $M$ determines an orientation of $M_{\tilde{F}}^{\epsilon}$ by restriction, and this orientation of $M_{\tilde{F}}^{\epsilon}$ induces an orientation of $\tilde{F}_{\epsilon}$. The two orientations of $\tilde{F}_{\epsilon}$ which arise in this way are identified with the two consistent orientations of $\tilde{F}$.

5.2. Supports of reduced homotopies. Throughout this subsection we shall assume that $M$ is a simple knot manifold, and that $\tilde{F}$ is a splitting surface in $M$.

5.2.1. It follows from the characteristic submanifold theory of Jaco-Shalen JS and Johannson [Jo] that for each $\epsilon \in\{ \pm 1\}$ there is an $(I, \partial I)$-bundle pair $\left(\Sigma^{\epsilon}, \Phi^{\epsilon}\right) \subset$ $\left(M_{\tilde{F}}^{\epsilon}, \tilde{F}\right)$, well defined up to ambient isotopy in $\left(M_{\tilde{F}}^{\epsilon}, \tilde{F}\right)$, such that

(1) the frontier of $\Sigma^{\epsilon}$ in $M_{\tilde{F}}^{\epsilon}$ consists of essential annuli in $\left(M_{\tilde{F}}^{\epsilon}, \tilde{F}\right)$;

(2) no component $(\sigma, \phi)$ of $\left(\Sigma^{\epsilon}, \Phi^{\epsilon}\right)$ is homotopic (as a pair) into $\left(\Sigma^{\epsilon}-\sigma, \Phi^{\epsilon}-\phi\right)$; and

(3) if $K$ is a polyhedron and $H:(K \times I, K \times \partial I) \rightarrow\left(M_{\tilde{F}}^{\epsilon}, \tilde{F}\right)$ is an essential basic homotopy such that $H_{0}: K \rightarrow \tilde{F}$ is a large map, then $H$ is homotopic as a map of pairs to a homotopy whose image lies in $\Sigma^{\epsilon}$.

The $I$-fibers of the $I$-bundle structure on $\Sigma^{\epsilon}$ can be used to build an essential basic homotopy of $\Phi^{\epsilon}$ in $\Sigma^{\epsilon}$. Indeed, define a fundamental homotopy of $\Phi^{\epsilon}$ to be any essential basic homotopy

$$
H_{\Sigma^{\epsilon}}:\left(\Phi^{\epsilon} \times I, \Phi^{\epsilon} \times \partial I\right) \rightarrow\left(\Sigma^{\epsilon}, \Phi^{\epsilon}\right) \subset\left(M_{\tilde{F}}^{\epsilon}, \tilde{F}\right)
$$

satisfying the following conditions:

(1) The time-0 map of $H_{\Sigma^{\epsilon}}$ is the identity map of $\Phi^{\epsilon}$.

(2) For each $x \in \Phi^{\epsilon}, H_{\Sigma^{\epsilon}}(\{x\} \times I)$ is an $I$-fiber of $\Sigma^{\epsilon}$.

(3) For each component $\phi$ of $\Phi^{\epsilon}$ which is contained in a trivial $I$-bundle component $\sigma$ of $\Sigma^{\epsilon}$, the map $H_{\Sigma^{\epsilon}}$ restricts to a homeomorphism of $\phi \times I$ onto $\sigma$.

(4) For each component $\phi$ of $\Phi^{\epsilon}$ which is contained in a non-trivial $I$-bundle component $\sigma$ of $\Sigma^{\epsilon}$, the map $H_{\Sigma^{\epsilon}}$ restricts to a 2-fold covering map from $\phi \times I$ to $\sigma$. 
It is clear that a fundamental homotopy exists and is unique up to composition with a fiber-preserving homeomorphism of $\Sigma^{\epsilon}$ which restricts to the identity on $\Phi^{\epsilon}$. Note also that a fundamental homotopy is a two-sheeted covering map from $\Phi^{\epsilon} \times I$ to $\Sigma^{\epsilon}$. The involution of $\Phi^{\epsilon}$ induced by its $\partial I$-bundle structure is the restriction of the deck transformation of this two-sheeted covering map. This involution will be denoted by $\tau_{\epsilon}$. Thus

$$
H_{\Sigma^{\epsilon}}(x, 1)=\tau_{\epsilon}(x)
$$

for every $x \in \Phi^{\epsilon}$.

The notation $\Sigma^{\epsilon}, \Phi^{\epsilon}$ and $\tau_{\epsilon}$ will be used throughout this section.

Lemma 5.2.2. Let $\tilde{F}$ be given a consistent orientation. Then the embedding $\tau_{\epsilon}$ : $\Phi^{\epsilon} \rightarrow \tilde{F}$ reverses orientation. (See Subsection 1.1.)

Proof. By Subsection 5.1, the orientation of $\Phi^{\epsilon}$ is induced from an orientation of $M_{\tilde{F}}^{\epsilon}$. Hence, if we orient $\Sigma^{\epsilon}$ by restricting the orientation of $M_{\tilde{F}}^{\epsilon}$, then the orientation of $\Phi^{\epsilon}$ is induced by the orientation of $\Sigma^{\epsilon}$. The involution $\tau_{\epsilon}$ extends to an involution $\sigma$ of $\Sigma^{\epsilon}$ which leaves each $I$-fiber invariant while reversing its orientation. Since $\sigma$ clearly reverses the orientation of $\Sigma^{\epsilon}$, it follows that $\tau_{\epsilon}$ reverses the orientation of $\Phi^{\epsilon}$.

Definition 5.2.3. An essential basic homotopy $H:(K \times I, K \times \partial I) \rightarrow\left(\Sigma^{\epsilon}, \Phi^{\epsilon}\right)$ is said to be standard if it is of the form $H(x, t)=H_{\Sigma^{\epsilon}}(f(x), t)$, where $H_{\Sigma^{\epsilon}}$ is a fundamental homotopy and $f$ is some map from $K$ to $\Phi^{\epsilon}$. Note that we then have $H_{0}=f$. Note also that if $f^{\prime}: K \rightarrow \Phi^{\epsilon}$ is homotopic to $f$ in $\Phi^{\epsilon}$, then the standard homotopies $H(x, t)=H_{\Sigma^{\epsilon}}(f(x), t)$ and $H^{\prime}(x, t)=H_{\Sigma^{\epsilon}}\left(f^{\prime}(x), t\right)$ are homotopic as maps of pairs $(K \times I, K \times \partial I) \rightarrow\left(\Sigma^{\epsilon}, \Phi^{\epsilon}\right)$.

Lemma 5.2.4. If $H:(K \times I, K \times \partial I) \rightarrow\left(M_{\tilde{F}}^{\epsilon}, \tilde{F}\right)$ is an essential basic homotopy such that $H_{0}: K \rightarrow \tilde{F}$ is a large map, then $H$ is homotopic as a map of pairs to a standard essential basic homotopy.

Proof. Appealing to the characteristic submanifold theory [JS], JJo], we may assume, without loss of generality, that $H(K \times I) \subset \Sigma^{\epsilon}$ and $H(K \times \partial I) \subset \Phi^{\epsilon}$. It is not hard to see that $H$ lifts to a map $\tilde{H}:(K \times I, K \times \partial I) \rightarrow\left(\Phi^{\epsilon} \times I, \Phi^{\epsilon} \times\{0,1\}\right)$ such that $H=H_{\Sigma^{\epsilon}} \circ \tilde{H}$. Write $\tilde{H}(x, t)=\left(H^{\prime}(x, t), T(x, t)\right) \in \Phi^{\epsilon} \times I$ and observe that $T(x, 0)=0$ for each $x \in K$, while the essentiality of $H$ implies that $T(x, 1)=1$ for each $x$.

Let $J:((K \times I) \times I,(K \times I) \times\{0,1\}) \rightarrow\left(\Sigma^{\epsilon}, \Phi^{\epsilon}\right)$ be given by

$$
J((x, t), s)=H_{\Sigma^{\epsilon}}\left(H^{\prime}(x,(1-s) t),(1-s) T(x, t)+s t\right) .
$$

Then

$$
\begin{aligned}
& J((x, t), 0)=\left(H_{\Sigma^{\epsilon}} \circ \tilde{H}\right)(x, t)=H(x, t), \text { and } \\
& J((x, t), 1)=H_{\Sigma^{\epsilon}}\left(H^{\prime}(x, 0), t\right)=H_{\Sigma^{\epsilon}}\left(H_{0}(x), t\right),
\end{aligned}
$$

while for each $x \in K$,

$$
\begin{aligned}
& J((x, 0), s)=H(x, 0) \in \Phi^{\epsilon}, \text { and } \\
& J((x, 1), s)=H_{\Sigma^{\epsilon}}\left(H^{\prime}(x, 1-s), 1\right) \in \Phi^{\epsilon} .
\end{aligned}
$$

Thus $H=J_{0}$ is homotopic as a map of pairs to the standard essential basic homotopy $J_{1}$. 
5.2.5. We define $\Phi_{1}^{\epsilon}=\mathcal{L}\left(\Phi^{\epsilon}\right) \subset \tilde{F}$. Note that the free involution $\tau_{\epsilon}$ restricts to a free involution of $\Phi_{1}^{\epsilon}$. We shall denote this restriction by $\tau_{\epsilon}$ as well.

Lemma 5.2.6. The surface $\Phi_{1}^{\epsilon}$ has the following property.

For any large map $f: K \rightarrow \tilde{F}$, there exists an essential basic homotopy $H$ in the pair $(M, \tilde{F})$ on the $\epsilon$-side with $H_{0}=f$ if and only if $f$ is homotopic in $\tilde{F}$ to a map with image in $\Phi_{1}^{\epsilon}$.

Furthermore, any large subsurface of $F$ with this property is isotopic to $\Phi_{1}^{\epsilon}$.

Proof. If $f: K \rightarrow \tilde{F}$ is a large map homotopic in $\tilde{F}$ to some $f^{\prime}$ for which $f^{\prime}(K) \subset \Phi_{1}^{\epsilon}$ and $H_{\Sigma^{\epsilon}}:\left(\Phi^{\epsilon} \times[0,1], \Phi^{\epsilon} \times\{0,1\}\right) \rightarrow\left(\Sigma^{\epsilon}, \Phi^{\epsilon}\right)$ is a fundamental homotopy, then $H:(K \times[0,1], K \times\{0,1\}) \rightarrow\left(\Sigma^{\epsilon}, \Phi^{\epsilon}\right) \subset\left(M_{\tilde{F}}^{\epsilon}, \tilde{F}\right)$ defined by

$$
H(x, t)=H_{\Sigma^{\epsilon}}\left(f^{\prime}(x), t\right)
$$

is an essential basic homotopy in $(M, \tilde{F})$ on the $\epsilon$-side, with $H_{0}=f^{\prime}$. The desired essential basic homotopy with time- 0 map $f$ is now readily constructed.

Conversely let $H:(K \times[0,1], K \times\{0,1\}) \rightarrow(M, \tilde{F})$ be an essential basic homotopy in $(M, \tilde{F})$ on the $\epsilon$-side with $H_{0}=f$. According to Subsection 3.1, any essential basic homotopy in $(M, \tilde{F})$ which starts on the $\epsilon$-side corresponds to an essential homotopy in the pair $\left(M_{\tilde{F}}^{\epsilon}, \tilde{F}\right)$. So it follows from Lemma 5.2.4 that $H$ is homotopic as a map of pairs to a standard essential basic homotopy $H^{\prime}$. It follows from Subsection 5.2.1 and Definition 5.2.3 that $H_{t}^{\prime}(K) \subset \Phi^{\epsilon}$ for $t=0,1$, that $H_{0}^{\prime}$ is homotopic to $f$ and that $H_{1}^{\prime}=\tau_{\epsilon} \circ H_{0}^{\prime}$. Since $f$, and hence $H_{0}^{\prime}$, is large, the image of $H_{0}^{\prime}$ is contained in $\Phi_{1}^{\epsilon}$.

Finally suppose that $\Psi_{1}^{\epsilon} \subset \tilde{F}$ is another large subsurface of $\tilde{F}$ which has the stated property. Then the inclusion $\Phi_{1}^{\epsilon} \rightarrow \tilde{F}$ is homotopic into $\Psi_{1}^{\epsilon}$ and vice versa. Thus by Lemma $4.1, \Psi_{1}^{\epsilon}$ is isotopic to $\Phi_{1}^{\epsilon}$ in $\tilde{F}$.

Lemma 5.2.7. Let $H:(K \times I, K \times \partial I) \rightarrow(M, \tilde{F})$ be an essential basic homotopy on the $\epsilon$-side such that $H_{0}$ is a large map from $K$ to $\tilde{F}$. If $f: K \rightarrow \Phi_{1}^{\epsilon}$ is any map which is homotopic in $\tilde{F}$ to $H_{0}$, then $H_{1}$ is homotopic in $\tilde{F}$ to $\tau_{\epsilon} \circ f$.

Proof. By Lemma 5.2.4 the homotopy $H$ is homotopic as a map of pairs to a standard essential basic homotopy $H^{\prime}$. By Proposition $4.4 H_{0}^{\prime}$ is homotopic to $f$ in $\Phi_{1}^{\epsilon}$ and so by the remark at the end of Definition 5.2.3 we may assume that $H_{0}^{\prime}=f$. Thus $H_{1}$ is homotopic in $\tilde{F}$ to $H_{1}^{\prime}=\tau_{\epsilon} \circ f$.

Our next goal is to extend Lemma 5.2.6 to reduced homotopies of length $n$ in $(M, \tilde{F})$.

Proposition 5.2.8. For each fixed $\epsilon \in\{ \pm 1\}$, there is a sequence of large (possibly empty) subsurfaces $\left(\Phi_{k}^{\epsilon}\right)_{k \geq 0}$ of $\tilde{F}$, such that $\Phi_{0}^{\epsilon}=\tilde{F}$, $\Phi_{1}^{\epsilon}$ is the surface defined in Subsection 5.2.5, and for each $k \geq 0$ we have:

(1) $\Phi_{k}^{\epsilon} \supset \Phi_{k+1}^{\epsilon}$; and

(2) a large map $f: K \rightarrow \tilde{F}$ is homotopic in $\tilde{F}$ to a map with image in $\Phi_{k}^{\epsilon}$ if and only if there exists a reduced homotopy $H$ of length $k$ starting on the $\epsilon$-side with $H_{0}=f$.

Furthermore condition (2) determines $\Phi_{k}^{\epsilon}$, up to isotopy, among the class of large subsurfaces of $\tilde{F}$. 
Proof. We construct the surfaces inductively in such a way that (1) and (2) hold. Set $\Phi_{0}^{\epsilon}=\tilde{F}$ and let $\Phi_{1}^{\epsilon}$ be defined as in Subsection 5.2.5. According to Lemma 5.2.6, condition (2) holds for $k=1$. Let $m \geq 2$ be given, and suppose that for $\epsilon= \pm 1$ we have defined large subsurfaces

$$
\tilde{F}=\Phi_{0}^{\epsilon} \supset \Phi_{1}^{\epsilon} \supset \Phi_{2}^{\epsilon} \supset \cdots \supset \Phi_{m-1}^{\epsilon}
$$

such that condition (2) holds for $k<m$ and for $\epsilon= \pm 1$. As we observed in Definition 4.3, there is a surface $A_{m}^{\epsilon} \subset \Phi_{1}^{\epsilon}$ which is isotopic to the large intersection $\Phi_{1}^{\epsilon} \wedge_{\mathcal{L}} \Phi_{m-1}^{-\epsilon}$. For $\epsilon= \pm 1$ we define

$$
\Psi_{m}^{\epsilon}=\tau_{\epsilon}\left(A_{m}^{\epsilon}\right) .
$$

Note that by Subsection 5.2.5, $\Phi_{1}^{\epsilon}$ is invariant under the map $\tau_{\epsilon}$ and so we have

$$
\Psi_{m}^{\epsilon} \subset \Phi_{1}^{\epsilon}
$$

For $\epsilon= \pm 1$, we claim that:

(*) a large map $f: K \rightarrow \tilde{F}$ is homotopic in $\tilde{F}$ to a map with image in $\Psi_{m}^{\epsilon}$ if and only if there exists a reduced homotopy $H$ of length $m$ starting on the $\epsilon$-side with $H_{0}=f$.

To prove this, we first consider a large map $f: K \rightarrow \tilde{F}$ and assume that there exists a reduced homotopy $H: K \times I \rightarrow(M, \tilde{F})$ of length $m$ starting on the $\epsilon$ side with $H_{0}=f$. Write $H$ as a composition of $m$ essential basic homotopies $H^{1}, \ldots, H^{m}$, where $H^{i}$ starts on the $(-1)^{i-1} \epsilon$ side for $i=1, \ldots, m$. Define $f_{1}: K \rightarrow$ $\tilde{F}$ by $f_{1}=H_{1}^{1}=H_{0}^{2}$ and let $H^{\prime}$ be the composition of $H^{2}, \ldots, H^{m}$. Then $f_{1}$ is large and $H^{\prime}$ is a reduced homotopy of length $m-1$ starting on the $-\epsilon$-side, with $H_{0}^{\prime}=f_{1}$. Our inductive hypothesis implies that $f_{1}$ is homotopic to a map with image in $\Phi_{m-1}^{-\epsilon}$. On the other hand, $H^{1}$ is an essential basic homotopy on the $\epsilon$ side with $H_{0}^{1}=f$ and $H_{1}^{1}=f_{1}$; hence by Lemma 5.2.7 we see that $f_{1}$ is homotopic in $\tilde{F}$ to a map $f_{1}^{\prime}$ whose image lies in $\Phi_{1}^{\epsilon}$, and that $f$ is homotopic in $\tilde{F}$ to $\tau_{\epsilon} \circ f_{1}^{\prime}$. Proposition 4.2 now implies that $f_{1}^{\prime}$ is homotopic in $\tilde{F}$ to a map $f_{1}^{\prime \prime}$ whose image lies in $A_{m}^{\epsilon}$. Proposition 4.4 implies that $f_{1}^{\prime}$ is homotopic to $f_{1}^{\prime \prime}$ in $\Phi_{1}^{\epsilon}$. Composing this homotopy with $\tau_{\epsilon}$ we see that $f$ is homotopic to a map with image in $\tau_{\epsilon}\left(A_{m}^{\epsilon}\right)=\Psi_{m}^{\epsilon}$.

To prove the converse observe that the fundamental homotopy

$$
H=H_{\Sigma^{\epsilon}}:\left(\Phi^{\epsilon} \times[0,1], \Phi^{\epsilon} \times\{0,1\}\right) \rightarrow\left(\Sigma^{\epsilon}, \Phi^{\epsilon}\right)
$$

is an essential basic homotopy $H$ in $(M, \tilde{F})$ on the $\epsilon$-side such that $H_{1}$ is the inclusion $i_{0}: \Phi_{1}^{\epsilon} \rightarrow \tilde{F}$, and $H_{1}=\tau_{\epsilon} \mid \Phi_{1}^{\epsilon}$. Since $\Psi_{m}^{\epsilon} \subset \Phi_{1}^{\epsilon}$, we may restrict $H$ to $\Psi_{m}^{\epsilon} \times I$ to obtain an essential basic homotopy $H^{1}$ in $(M, \tilde{F})$ on the $\epsilon$-side. The time- 0 map of $H^{1}$ is the inclusion $\Psi_{m}^{\epsilon} \rightarrow \tilde{F}$ and the time-1 map of $H^{1}$ is a homeomorphism $\psi: \Psi_{m}^{\epsilon} \rightarrow A_{m}^{\epsilon}$. By Proposition 4.2 and Definition 4.3, $A_{m}^{\epsilon}$ is isotopic to a subsurface of $\Phi_{m-1}^{-\epsilon}$. Since our induction hypothesis implies that the inclusion $\Phi_{m-1}^{-\epsilon} \rightarrow \tilde{F}$ is the time-0 map of a reduced homotopy of length $m-1$ starting on the $-\epsilon$ side, it follows that $\psi$ is also the time- 0 map of a reduced homotopy $H^{\prime}$ in $(M, \tilde{F})$ of length $m-1$ starting on the $-\epsilon$-side. The composition of the two homotopies $H_{1}$ and $H^{\prime}$ is a reduced homotopy of length $m$ starting on the $\epsilon$-side whose time- 0 map is the inclusion $\Psi_{m}^{\epsilon} \rightarrow \tilde{F}$. This completes the proof of $(*)$.

It follows from $(*)$ that there is a reduced homotopy of length $m$ starting on the $\epsilon$-side with time-0 map equal to the inclusion map of $\Psi_{m}^{\epsilon}$ into $\tilde{F}$. Clearly there also exists a reduced homotopy of length $m-1$ starting on the same side and having 
the same time-0 map. Thus we may apply property (2) again to conclude that the inclusion map of $\Psi_{m}^{\epsilon}$ is homotopic to a map with image in $\Phi_{m-1}^{\epsilon}$. By Lemma 4.1, $\Psi_{m}^{\epsilon}$ is isotopic to a subsurface $\Phi_{m}^{\epsilon}$ of $\Phi_{m-1}^{\epsilon}$. It follows from (*) that condition (2) holds for $k=m$. The induction is now complete.

To prove the last assertion of the proposition, suppose that $\Psi_{k}^{\epsilon} \subset \tilde{F}$ is another large surface in $\tilde{F}$ which satisfies condition (2) of the theorem. Taking $f$ to be the inclusion map of either surface into $\tilde{F}$, we see that $\Phi_{k}^{\epsilon}$ is homotopic into $\Psi_{k}^{\epsilon}$ and vice versa. Thus by Lemma $4.1, \Psi_{k}^{\epsilon}$ is isotopic to $\Phi_{k}^{\epsilon}$ in $\tilde{F}$.

5.3. Time-1 maps of reduced homotopies. Throughout this section $M$ will denote a simple knot manifold and $\tilde{F}$ will denote a splitting surface in $M$. We will define $\Phi^{\epsilon}$ and $\tau_{\epsilon}$ as in Subsection 5.2. We shall also fix subsurfaces $\Phi_{k}^{\epsilon}$ of $\tilde{F}$ for which the conclusions of Proposition 5.2.8 hold. A crucial ingredient in obtaining a bound of the type given by Theorem 5.4.1 is provided by Proposition 5.3.9, which asserts that if the inclusion $\Phi_{k+2}^{\epsilon} \subseteq \Phi_{k}^{\epsilon}$ is a homotopy equivalence, then $\tilde{F}$ is a semi-fiber. The estimate is strengthened by using Proposition 5.3.7, which asserts that for each odd $k, \Phi_{k}^{\epsilon}$ admits a fixed-point free involution.

Proposition 5.3.1. For each $\epsilon \in\{ \pm 1\}$ and each $k \geq 0$ there exists a homeomorphism $h_{k}^{\epsilon}: \Phi_{k}^{\epsilon} \rightarrow \Phi_{k}^{(-1)^{k+1} \epsilon}$ which has the following property:

(*) For any reduced homotopy $H$ of length $k$ with domain $K$ which starts on the $\epsilon$ side and has a large time-0 map, there exists a map $f: K \rightarrow \Phi_{k}^{\epsilon}$ such that $H_{0}$ is homotopic in $\tilde{F}$ to $f$ and $H_{1}$ is homotopic in $\tilde{F}$ to $h_{k}^{\epsilon} \circ f$.

The homeomorphism $h_{k}^{\epsilon}$ is unique up to isotopy. Furthermore, if $\tilde{F}$ is given a consistent orientation, then the embedding $h_{k}^{\epsilon}: \Phi_{k}^{\epsilon} \rightarrow \tilde{F}$ reverses orientation if $k$ is odd and preserves orientation if $k$ is even. (See Subsection 1.1.) We may take $h_{1}^{\epsilon}$ to be the map $\tau_{\epsilon} \mid \Phi_{1}^{\epsilon}$ described in Subsection 5.2.5.

Proof. We construct the maps $h_{k}^{\epsilon}$ by induction on $k$. For each $\epsilon \in\{ \pm 1\}$ define $h_{0}^{\epsilon}$ to be the identity map of $\tilde{F}$ and $h_{1}^{\epsilon}$ to be the map $\tau_{\epsilon}$. It is clear that property (*) holds for $h_{0}^{\epsilon}$ and Lemmas 5.2.6 and 5.2.7 imply that it holds for $h_{1}^{\epsilon}$.

Suppose that for $\epsilon= \pm 1$ we have constructed a homeomorphism $h_{k}^{\epsilon}: \Phi_{k}^{\epsilon} \rightarrow$ $\Phi_{k}^{(-1)^{k+1} \epsilon}$ with property $(*)$. In particular, since $\Phi_{k}^{\epsilon}$ is a $\pi_{1}$-injective subsurface, $h_{k}^{\epsilon}$ is a $\pi_{1}$-injective map. By Proposition 5.2.8, for $\epsilon= \pm 1$, we may choose a reduced homotopy $\mathcal{H}$ of length $k+1$ starting on the $\epsilon$ side such that $\mathcal{H}_{0}$ is the inclusion $\Phi_{k+1}^{\epsilon} \rightarrow \tilde{F}$. Write $\mathcal{H}$ as the composition of a reduced homotopy $\mathcal{H}^{\prime}$ of length $k$ and an essential basic homotopy $\mathcal{H}^{\prime \prime}$. The induction hypothesis implies that the time-1 map of $\mathcal{H}^{\prime}$ is homotopic to the embedding $h_{k}^{\epsilon} \mid \Phi_{k+1}^{\epsilon}$. By reversing the time variable of $\mathcal{H}^{\prime}$ and applying Proposition 5.2 .8 we see that $h_{k}^{\epsilon} \mid \Phi_{k+1}^{\epsilon}$ is homotopic to an embedding $h: \Phi_{k+1}^{\epsilon} \rightarrow \Phi_{k}^{(-1)^{k+1} \epsilon} \subset \Phi_{1}^{(-1)^{k+1} \epsilon}$. We define $\theta^{\epsilon}: \Phi_{k+1}^{\epsilon} \rightarrow \tilde{F}$ to be $\tau_{(-1)^{k+1} \epsilon} \circ h$. Note that $h$ is $\pi_{1}$-injective since $\Phi_{k+1}^{\epsilon}$ is a $\pi_{1}$-injective subsurface and since $h_{k}^{\epsilon}$ is a $\pi_{1}$-injective map. Furthermore since $\tau_{(-1)^{k+1} \epsilon}$ is an involution of a $\pi_{1}$-injective subsurface, it follows that $\theta^{\epsilon}$ is a $\pi_{1}$-injective map for $\epsilon= \pm 1$.

Since $\tau_{(-1)^{k+1} \epsilon}$ is an involution of $\Phi_{1}^{(-1)^{k+1} \epsilon}$ we know that $\theta^{\epsilon}$ is the time- 0 map of a standard basic essential homotopy whose time-1 map is $\tau_{(-1)^{k+1}{ }_{\epsilon}} \circ \theta^{\epsilon}=h$. Since $h$ is in turn the time- 0 map of a reduced homotopy of length $k$ starting on the $(-1)^{k+1} \epsilon-$ side, it follows that $\theta^{\epsilon}$ is the time- 0 map of a length $k+1$ homotopy starting on 
the $(-1)^{k} \epsilon$-side. It thus follows from Proposition 5.2.8 that the embedding $\theta^{\epsilon}$ is homotopic into $\Phi_{k+1}^{(-1)^{k} \epsilon}$, i.e. that the surface $\theta^{\epsilon}\left(\Phi_{k+1}^{\epsilon}\right)$ is homotopic into $\Phi_{k+1}^{(-1)^{k} \epsilon}$.

Replacing $\epsilon$ by $(-1)^{k} \epsilon$ we find that $\theta^{(-1)^{k} \epsilon}\left(\Phi_{k+1}^{(-1)^{k} \epsilon}\right)$ is homotopic into $\Phi_{k+1}^{\epsilon}$. By Lemma 4.1(1), $\theta^{(-1)^{k} \epsilon}\left(\Phi_{k+1}^{(-1)^{k} \epsilon}\right)$ is isotopic to a subsurface of $\Phi_{k+1}^{\epsilon}$, which is $\pi_{1}$ injective since $\theta^{(-1)^{k} \epsilon}$ is a $\pi_{1}$-injective map. In particular $\Phi_{k+1}^{(-1)^{k} \epsilon}$ is homeomorphic to a $\pi_{1}$-injective subsurface of $\Phi_{k+1}^{\epsilon}$, and hence to a $\pi_{1}$-injective subsurface of $\theta^{\epsilon}\left(\Phi_{k+1}^{\epsilon}\right)$. Apply Lemma 4.1(2), taking $A=\theta^{\epsilon}\left(\Phi_{k+1}^{\epsilon}\right)$ and $B=\Phi_{k+1}^{(-1)^{k} \epsilon}$. It follows that $A$ and $B$ are isotopic and hence that $\theta^{\epsilon}$ is isotopic in $\tilde{F}$ to a homeomorphism $h_{k+1}^{\epsilon}: \Phi_{k+1}^{\epsilon} \rightarrow \Phi_{k+1}^{(-1)^{k} \epsilon}$.

We now show that $h_{k+1}^{\epsilon}$ has property $(*)$. Let $H$ be any reduced homotopy of length $k+1$ starting on the $\epsilon$ side such that $H_{0}$ is a large map. By Proposition 5.2.8, $H_{0}$ is homotopic in $\tilde{F}$ to a map $f: K \rightarrow \Phi_{k+1}^{\epsilon}$. Write $H$ as the composition of a reduced homotopy $H^{\prime}$ of length $k$ and a basic essential homotopy $H^{\prime \prime}$. Since $h_{k}^{\epsilon}$ has property $(*)$, the map $H_{0}^{\prime \prime}=H_{1}^{\prime}$ is homotopic in $\tilde{F}$ to $h_{k}^{\epsilon} \circ f$, which is in turn homotopic to $h \circ f$. Then Lemma 5.2.7 implies that $H_{1}=H_{1}^{\prime \prime}$ is homotopic to $\tau_{(-1)^{k} \epsilon} \circ h \circ f=\theta^{\epsilon} \circ f$, and hence to $h_{k+1}^{\epsilon} \circ f$. This establishes $(*)$ and completes the inductive definition of the $h_{j}^{\epsilon}$.

By Lemma 5.2.2, if $\tilde{F}$ is given a consistent orientation, the involutions $\tau_{ \pm 1}$ are orientation reversing embeddings of $\phi_{ \pm 1}$ into $\tilde{F}$. It follows from the inductive construction that $h_{k}^{ \pm 1}$ reverses orientation if $k$ is odd and preserves orientation if $k$ is even.

It remains to prove that a homeomorphism satisfying $(*)$ is unique up to isotopy. Suppose that $h, h^{\prime}: \Phi_{k}^{\epsilon} \rightarrow \Phi_{k}^{(-1)^{k+1} \epsilon}$ both satisfy (*). We apply Proposition 5.2.8, taking $K=\Phi_{k}^{\epsilon}$ and taking $f: \Phi_{k}^{\epsilon} \rightarrow \tilde{F}$ to be the inclusion. This gives a length- $k$ reduced homotopy $H$ starting on the $\epsilon$ side with time- 0 map $f$. By property $(*)$, $h$ and $h^{\prime}$ are both homotopic in $\tilde{F}$ to $H_{1}$, and hence to each other. By Proposition $4.4, h$ and $h^{\prime}$ are homotopic as maps from $h, h^{\prime}: \Phi_{k}^{\epsilon}$ to $\Phi_{k}^{(-1)^{k+1} \epsilon}$. It follows from Theorem 6.4 and Theorem A.4 of [E] that they are isotopic.

For the rest of this section, it will be understood that for each $k$ and each $\epsilon$ we have fixed homeomorphisms $h_{k}^{\epsilon}: \Phi_{k}^{\epsilon} \rightarrow \Phi_{k}^{(-1)^{k+1} \epsilon}$ for which condition (*) of Proposition 5.3.1 holds.

Lemma 5.3.2. Let $\epsilon \in\{ \pm 1\}$ and $k \geq 0$ be given. Suppose that $H$ is a reduced homotopy in $(M, \tilde{F})$ of length $k$ with domain $K$ which starts on the $\epsilon$ side. Supppose that $f=H_{0}$ is large and that $f(K) \subset \Phi_{k}^{\epsilon}$. Then $H_{1}$ is homotopic in $\tilde{F}$ to $h_{k}^{\epsilon} \circ f$.

Proof. By condition $(*)$ of 5.3.1 there is a map $f^{\prime}: K \rightarrow \Phi_{k}^{\epsilon}$ such that $H_{0}=f$ is homotopic in $\tilde{F}$ to $f^{\prime}$ and such that $H_{1}$ is homotopic in $\tilde{F}$ to $h_{k}^{\epsilon} \circ f^{\prime}$. It follows from Proposition 4.4 that $f$ is homotopic to $f^{\prime}$ in $\Phi_{k}^{\epsilon}$. Thus $h_{k}^{\epsilon} \circ f$ is homotopic in $\tilde{F}$ to $h_{k}^{\epsilon} \circ f^{\prime}$, and hence to $H_{1}$.

Proposition 5.3.3. Let $\epsilon \in\{ \pm 1\}$ and $k \geq 0$ be given. Then the homeomorphism $h_{k}^{(-1)^{k+1} \epsilon}: \Phi_{k}^{(-1)^{k+1} \epsilon} \rightarrow \Phi_{k}^{\epsilon}$ is isotopic to the inverse of $h_{k}^{\epsilon}: \Phi_{k}^{\epsilon} \rightarrow \Phi_{k}^{(-1)^{k+1} \epsilon}$.

Proof. It follows from Proposition 5.2.8 that there is a reduced homotopy $H$ of length $k$ starting on the $\epsilon$-side such that $H_{0}$ is the inclusion $\iota: \Phi_{k}^{\epsilon} \rightarrow \tilde{F}$. By Lemma 
5.3.2 we have that $H_{1}$ is homotopic in $\tilde{F}$ to $h_{k}^{\epsilon}$. Applying Lemma 5.3.2 to the homotopy $H^{\prime}$ obtained by reversing the time variable of $H$, we see that $\iota=H_{0}=H_{1}^{\prime}$ is homotopic in $\tilde{F}$ to the composition $h_{k}^{(-1)^{k+1} \epsilon} \circ H_{0}^{\prime}$. Since $H_{0}^{\prime}=H_{1} \sim h_{k}^{\epsilon}$ we have that $h_{k}^{(-1)^{k+1} \epsilon} \circ h_{k}^{\epsilon}$ is homotopic in $\tilde{F}$ to the inclusion $\iota$. It now follows from Proposition 4.4 that the self-homeomorphism $h_{k}^{(-1)^{k+1} \epsilon} \circ h_{k}^{\epsilon}$ of $\Phi_{k}^{\epsilon}$ is homotopic to the identity in $\Phi_{k}^{\epsilon}$, and therefore isotopic to the identity by Theorem 6.4 and Theorem A.4 of [E].

Proposition 5.3.4. Let $i$ and $j$ be non-negative integers, and set $k=i+j$. Then for each $\epsilon \in\{ \pm 1\}$, the map $h_{i}^{\epsilon} \mid \Phi_{k}^{\epsilon}$ is homotopic in $\tilde{F}$ to an embedding $g_{i}^{\epsilon}: \Phi_{k}^{\epsilon} \rightarrow$ $\Phi_{j}^{(-1)^{i} \epsilon}$ such that $h_{j}^{(-1)^{i} \epsilon} \circ g_{i}^{\epsilon}$ is homotopic in $\tilde{F}$ to $h_{k}^{\epsilon}$.

Proof. Let $H$ be a reduced homotopy of length $k$ starting on the $\epsilon$ side such that $H_{0}$ is the inclusion $\Phi_{k}^{\epsilon} \rightarrow \tilde{F}$. Write $H$ as the composition of a reduced homotopy $H^{\prime}$ of length $i$ starting on the $\epsilon$ side and a reduced homotopy $H^{\prime \prime}$ of length $j$ starting on the $(-1)^{i} \epsilon$ side. Applying Lemma 5.3.2, with the roles of $k, H$ and $f$ played respectively by $i, H^{\prime}$ and the inclusion map $\Phi_{k}^{\epsilon} \rightarrow \Phi_{i}^{\epsilon}$, we find that $H_{1}^{\prime}=H_{0}^{\prime \prime}$ is homotopic to the embedding $h_{i}^{\epsilon} \mid \Phi_{k}^{\epsilon}$.

On the other hand it follows from Proposition 5.3.1 that $H_{0}^{\prime \prime}$ is homotopic in $\tilde{F}$ to a map $g_{i}^{\epsilon}: \Phi_{k}^{\epsilon} \rightarrow \Phi_{j}^{(-1)^{i} \epsilon}$. Since $g_{i}^{\epsilon}$ is homotopic to the embedding $h_{i}^{\epsilon} \mid \Phi_{k}^{\epsilon}$ it follows from part (1) of Lemma 4.1 that we may take $g_{i}^{\epsilon}$ to be an embedding. After modifying the homotopy $H$ we may assume that $H_{1}^{\prime}=H_{0}^{\prime \prime}=g_{i}^{\epsilon}$.

Applying Lemma 5.3.2 again, with $j, H^{\prime \prime}$ and $g_{i}^{\epsilon}$ playing the roles of $k, H$ and $f$, we conclude that $H_{1}=H_{1}^{\prime \prime}$ is homotopic in $\tilde{F}$ to $h_{j}^{(-1)^{i} \epsilon} \circ g_{i}^{\epsilon}$.

Finally, applying Lemma 5.3.2 directly to the homotopy $H$, we see that $H_{1}$ is homotopic in $\tilde{F}$ to $h_{k}^{\epsilon}$. The conclusion of the proposition follows.

Proposition 5.3.5. Let $i$ and $j$ be non-negative integers, and set $k=i+j$. Then for each $\epsilon \in\{ \pm 1\}$ the subsurface $h_{i}^{\epsilon}\left(\Phi_{k}^{\epsilon}\right)$ is isotopic in $\tilde{F}$ to $\Phi_{i}^{(-1)^{i+1} \epsilon} \wedge_{\mathcal{L}} \Phi_{j}^{(-1)^{i} \epsilon}$.

Proof. We have $h_{i}^{\epsilon}\left(\Phi_{k}^{\epsilon}\right) \subset h_{i}^{\epsilon}\left(\Phi_{i}^{\epsilon}\right)=\Phi_{i}^{(-1)^{i+1} \epsilon}$. On the other hand it follows from Proposition 5.3.4 that $h_{i}^{\epsilon}\left(\Phi_{k}^{\epsilon}\right)$ is isotopic in $\tilde{F}$ to a subsurface of $\Phi_{j}^{(-1)^{i} \epsilon}$. Hence by Definition 4.3 the subsurface $h_{i}^{\epsilon}\left(\Phi_{k}^{\epsilon}\right)$ is isotopic to a subsurface of $\Phi_{i}^{(-1)^{i+1} \epsilon} \wedge_{\mathcal{L}}$ $\Phi_{j}^{(-1)^{i} \epsilon}$.

To complete the proof of the proposition, it now suffices by Lemma 4.1(2) to show that the large surface $\Phi_{i}^{(-1)^{i+1} \epsilon} \wedge_{\mathcal{L}} \Phi_{j}^{(-1)^{i} \epsilon}$ is homeomorphic to a $\pi_{1}$-injective subsurface of $\Phi_{k}^{\epsilon}$ and hence of $h_{i}^{\epsilon}\left(\Phi_{k}^{\epsilon}\right)$. To prove this, note that $\Phi_{i}^{(-1)^{i+1} \epsilon} \wedge_{\mathcal{L}} \Phi_{j}^{(-1)^{i} \epsilon}$ is isotopic to a subsurface $A$ of $\Phi_{i}^{(-1)^{i+1} \epsilon}$. By Proposition 5.2.8 there is a reduced homotopy of length $i$ whose time-0 map is the inclusion $A \rightarrow \tilde{F}$ and whose time-1 map is homotopic to $h_{i}^{(-1)^{i+1} \epsilon}: A \rightarrow \tilde{F}$. By reversing the time variable we obtain a homotopy $H$ such that $H_{0}$ is homotopic in $\tilde{F}$ to $h_{i}^{(-1)^{i+1} \epsilon}: A \rightarrow \tilde{F}$, and $H_{1}$ is the inclusion $A \rightarrow \tilde{F}$. Since $A$ is isotopic to a subsurface of $\Phi_{j}^{(-1)^{i} \epsilon}$ there is also a reduced homotopy $H^{\prime \prime}$ of length $j$ starting on the $(-1)^{i} \epsilon$ side whose time-0 map is the inclusion $A \rightarrow \tilde{F}$. The composition of $H$ and $H^{\prime}$ is a reduced homotopy of length $k$ starting on the $\epsilon$ side and having time-0 map homotopic to $h_{i}^{(-1)^{i+1} \epsilon} \mid A: A \rightarrow \tilde{F}$. 
In particular, by Proposition 5.2.8 and Lemma 4.1, $h_{i}^{(-1)^{i+1} \epsilon}(A)$ is isotopic to a subsurface of $\Phi_{k}^{\epsilon}$.

One of the main results of this section is that $\Phi_{2 k+1}^{\epsilon}$ admits a free involution. The proof of this fact is based on our next lemma.

Lemma 5.3.6. The subsurface $h_{k}^{\epsilon}\left(\Phi_{2 k+1}^{\epsilon}\right)$ is isotopic in $\tilde{F}$ to a subsurface $A$ of $\Phi_{1}^{(-1)^{k} \epsilon}$ with the property that $\tau_{(-1)^{k} \epsilon}(A)$ is isotopic to $A$ in $\Phi_{1}^{(-1)^{k} \epsilon}$.

Proof. By Proposition 5.3.5 we have that $h_{k}^{\epsilon}\left(\Phi_{2 k+1}^{\epsilon}\right)$ is isotopic to $\Phi_{k}^{(-1)^{k+1} \epsilon} \wedge_{\mathcal{L}}$ $\Phi_{k+1}^{(-1)^{k} \epsilon}$. In particular, $h_{k}^{\epsilon}\left(\Phi_{2 k+1}^{\epsilon}\right)$ is isotopic to a subsurface $A$ of $\Phi_{k+1}^{(-1)^{k} \epsilon} \subset \Phi_{1}^{(-1)^{k} \epsilon}$. Since $\tau_{(-1)^{k} \epsilon}(A)=h_{1}^{(-1)^{k} \epsilon}(A) \subset h_{1}^{(-1)^{k} \epsilon}\left(\Phi_{k+1}^{(-1)^{k} \epsilon}\right)$, Proposition 5.3.4 implies that $\tau_{(-1)^{k} \epsilon}(A)$ is isotopic to a subsurface of $\Phi_{k}^{(-1)^{k+1} \epsilon}$. On the other hand, since $A$ is isotopic to a subsurface of $\Phi_{k}^{(-1)^{k+1} \epsilon}$ we know that the inclusion $A \rightarrow \tilde{F}$ is the time- 0 map of a reduced homotopy $H^{\prime \prime}$ of length $k$ starting on the $(-1)^{k+1} \epsilon$ side. Since $A \subset \Phi_{1}^{(-1)^{k+1} \epsilon}$, by reversing the time variable in the basic homotopy provided by Lemma 5.2 .7 we obtain a basic homotopy $H^{\prime}$ whose time- 0 map is the inclusion $\tau_{(-1)^{k} \epsilon} \mid A$ and whose time-1 map is the inclusion $A \rightarrow \tilde{F}$. The composition of $H^{\prime}$ and $H^{\prime \prime}$ is a reduced homotopy of length $k+1$ starting on the $(-1)^{k} \epsilon$ side whose time-0 map is $\tau_{(-1)^{k} \epsilon} \mid A$. It follows from Proposition 5.2.8 that $\tau_{(-1)^{k} \epsilon}(A)$ is homotopic into $\Phi_{k+1}^{(-1)^{k} \epsilon}$. By Definition 4.3 we have that $\tau_{(-1)^{k} \epsilon}(A)$ is homotopic into $\Phi_{k}^{(-1)^{k+1} \epsilon} \wedge_{\mathcal{L}} \Phi_{k+1}^{(-1)^{k} \epsilon}$ which in turn is isotopic to $A$ in $\tilde{F}$. By Lemma $4.1(2)$ and Proposition $4.4, \tau_{(-1)^{k} \epsilon}(A)$ is isotopic to $A$ in $\Phi_{1}^{(-1)^{k} \epsilon}$.

Proposition 5.3.7. The surface $h_{k}^{\epsilon}\left(\Phi_{2 k+1}^{\epsilon}\right)$ is isotopic in $\tilde{F}$ to a subsurface of $\Phi_{1}^{(-1)^{k} \epsilon}$ which is invariant under the free involution $\tau_{(-1)^{k} \epsilon}$. In particular, $\Phi_{2 k+1}^{\epsilon}$ admits a free involution.

Proof. This is an immediate consequence of Lemmas 5.3.6 and 4.5.

Corollary 5.3.8. For each odd integer $k>0$, the Euler characteristic $\chi\left(\Phi_{k}^{\epsilon}\right)$ is even.

Proposition 5.3.9. If $\Phi_{k}^{\epsilon}$ and $\Phi_{k+2}^{\epsilon}$ are isotopic in $\tilde{F}$ for a given $k \geq 0$, then either $\Phi_{k}^{\epsilon}=\emptyset$ or $\tilde{F}$ is a semi-fiber.

Proof. In this proof all isotopies will be understood to take place in $\tilde{F}$ unless specified otherwise.

First we claim that (1) $\Phi_{k}^{\epsilon}$ is isotopic to $\Phi_{m}^{\epsilon}$ for all $m \geq k$. Since $\Phi_{m-1}^{\epsilon} \supset \Phi_{m}^{\epsilon} \supset$ $\Phi_{m+1}^{\epsilon}$, and $\Phi_{m}^{\epsilon}$ is $\pi_{1}$-injective, it suffices to consider the case where $m-k$ is even. The case $m=k+2$ holds by hypothesis. Thus we need only show that if $m-k \geq 2$ is even and $\Phi_{m}^{\epsilon}$ is isotopic to $\Phi_{m-2}^{\epsilon}$, then $\Phi_{m+2}^{\epsilon}$ is isotopic to $\Phi_{m}^{\epsilon}$. By Proposition 5.3 .5 we have that $h_{2}^{\epsilon}\left(\Phi_{m+2}^{\epsilon}\right)$ and $h_{2}^{\epsilon}\left(\Phi_{m}^{\epsilon}\right)$ are respectively isotopic to $\Phi_{2}^{-\epsilon} \wedge_{\mathcal{L}} \Phi_{m}^{\epsilon}$ and $\Phi_{2}^{-\epsilon} \wedge_{\mathcal{L}} \Phi_{m-2}^{\epsilon}$. These two surfaces are isotopic by the induction hypothesis. It follows that $\Phi_{m+2}^{\epsilon}$ is isotopic to $\Phi_{m}^{\epsilon}$. Claim (1) follows.

Next we claim that $\left(1^{\prime}\right) \Phi_{m}^{-\epsilon}$ is isotopic to $\Phi_{k+1}^{-\epsilon}$ for all $m \geq k+1$. By Proposition 5.3.5 we have for any $m>k+1$ that $h_{1}^{-\epsilon}\left(\Phi_{m+1}^{-\epsilon}\right)$ is isotopic to $\Phi_{1}^{-\epsilon} \wedge_{\mathcal{L}} \Phi_{m}^{\epsilon}$, while 
$h_{1}^{-\epsilon}\left(\Phi_{k+1}^{-\epsilon}\right)$ is isotopic to $\Phi_{1}^{-\epsilon} \wedge_{\mathcal{L}} \Phi_{k}^{\epsilon}$. These two surfaces are isotopic by (1), so (1') follows.

Next we claim that (2) $h_{j}^{\epsilon}\left(\Phi_{k+j}^{\epsilon}\right)$ is isotopic to $\Phi_{k}^{\epsilon}$ for every even integer $j \geq 0$. By Proposition 5.3.5 we have that $h_{j}^{\epsilon}\left(\Phi_{k+j}^{\epsilon}\right)$ is isotopic to $\Phi_{j}^{-\epsilon} \wedge_{\mathcal{L}} \Phi_{k}^{\epsilon}$. In particular $h_{j}^{\epsilon}\left(\Phi_{k+j}^{\epsilon}\right)$ is isotopic to a subsurface of $\Phi_{k}^{\epsilon}$. Now since $\Phi_{k+j}^{\epsilon}$ is isotopic to $\Phi_{k}^{\epsilon}$ by (1), Lemma 4.1(2) implies that $h_{j}^{\epsilon}\left(\Phi_{k+j}^{\epsilon}\right)$ is isotopic to $\Phi_{k}^{\epsilon}$.

A similar argument using $\left(1^{\prime}\right)$ shows that $\left(2^{\prime}\right) h_{j}^{-\epsilon}\left(\Phi_{k+1+j}^{-\epsilon}\right)$ is isotopic to $\Phi_{k+1}^{-\epsilon}$ for all $j \geq 0$.

More generally we claim that (3) if $j$ is even and $l \geq \max (k, j)$, then $h_{j}^{\epsilon}\left(\Phi_{l}^{\epsilon}\right)$ is isotopic to $\Phi_{k}^{\epsilon}$. To show this we first note that by (1), $\Phi_{l}^{\epsilon}$ is isotopic in $\tilde{F}$ to $\Phi_{k+j}^{\epsilon}$ and so by Proposition $4.4 \Phi_{l}^{\epsilon}$ is isotopic to $\Phi_{k+j}^{\epsilon}$ in $\Phi_{j}^{\epsilon}$. Hence $h_{j}^{\epsilon}\left(\Phi_{l}^{\epsilon}\right)$ is isotopic in $\tilde{F}$ to $h_{k}^{\epsilon}\left(\Phi_{k+j}^{\epsilon}\right)$, which we have already shown is isotopic to $\Phi_{k}^{\epsilon}$.

In the same way, using $\left(2^{\prime}\right)$, we see that $\left(3^{\prime}\right)$ if $j$ is even and $l \geq \max (k+1, j)$, then $h_{j}^{-\epsilon}\left(\Phi_{l}^{-\epsilon}\right)$ is isotopic to $\Phi_{k+1}^{-\epsilon}$.

We claim that (4) for all $j \geq 0$ the surface $\Phi_{k}^{\epsilon}$ is isotopic to a subsurface of $\Phi_{j}^{-\epsilon}$ and that $\left(4^{\prime}\right) \Phi_{k+1}^{-\epsilon}$ is isotopic to a subsurface of $\Phi_{j}^{\epsilon}$. Since the $\Phi_{j}^{ \pm \epsilon}$ are nested up to isotopy, we need only prove this for even $j$. By (2) we have that $h_{j}^{\epsilon}\left(\Phi_{k+j}^{\epsilon}\right)$ is isotopic to $\Phi_{k}^{\epsilon}$. Lemma 5.3.5 implies that $h_{j}^{\epsilon}\left(\Phi_{k+j}^{\epsilon}\right)$ is isotopic to $\Phi_{j}^{-\epsilon} \wedge_{\mathcal{L}} \Phi_{k}^{\epsilon}$. The fact that $\Phi_{k}^{\epsilon}$ is isotopic to $\Phi_{j}^{-\epsilon} \wedge_{\mathcal{L}} \Phi_{k}^{\epsilon}$ implies that $\Phi_{k}^{\epsilon}$ is isotopic to a subsurface of $\Phi_{j}^{-\epsilon}$, as asserted by (4). A similar argument using $\left(2^{\prime}\right)$ proves $\left(4^{\prime}\right)$.

We next claim that (5) if $j$ is odd, then $h_{j}^{\epsilon}\left(\Phi_{k}^{\epsilon}\right)$ is isotopic to $\Phi_{k+1}^{-\epsilon}$ and $\left(5^{\prime}\right)$ if $j$ is odd, then $h_{j}^{-\epsilon}\left(\Phi_{k+1}^{-\epsilon}\right)$ is isotopic to $\Phi_{k}^{\epsilon}$. First by (1) we have that $h_{j}^{\epsilon}\left(\Phi_{k}^{\epsilon}\right)$ is isotopic to $h_{j}^{\epsilon}\left(\Phi_{k+1+j}^{\epsilon}\right)$ which, by Proposition 5.3.5, is isotopic to $\Phi_{j}^{\epsilon} \wedge_{\mathcal{L}} \Phi_{k+1}^{-\epsilon}$. Claim $\left(4^{\prime}\right)$ implies that this essential intersection is isotopic to $\Phi_{k+1}^{-\epsilon}$. The proof of $\left(5^{\prime}\right)$ is similar but uses $\left(1^{\prime}\right)$ and $(4)$ in place of $(1)$ and $\left(4^{\prime}\right)$.

Now fix an even integer $m>k$. By Proposition 5.3.7 we have that $h_{m}^{\epsilon}\left(\Phi_{2 m+1}^{\epsilon}\right)$ is isotopic to a $\tau_{\epsilon}$-invariant subsurface $B_{\epsilon}$ of $\Phi_{1}^{\epsilon}$. By (3) we have that $\Phi_{k}^{\epsilon}$ is isotopic to $h_{m}^{\epsilon}\left(\Phi_{2 m+1}^{\epsilon}\right)$ and hence to $B_{\epsilon}$. Similarly, using $\left(3^{\prime}\right)$, we see that $\Phi_{k+1}^{-\epsilon}$ is isotopic to a $\tau_{-\epsilon}$-invariant subsurface $B_{-\epsilon}$ of $\Phi_{1}^{-\epsilon}$. Moreover, since $\tau_{\epsilon}=h_{1}^{\epsilon}$, we have that $\Phi_{k}^{\epsilon}$ is isotopic to $\tau_{\epsilon}\left(\Phi_{k}^{\epsilon}\right)=h_{1}^{\epsilon}\left(\Phi_{k}^{\epsilon}\right)$ which in turn is isotopic to $\Phi_{k+1}^{-\epsilon}$ by (5).

Thus we have defined surfaces $B_{\epsilon} \subset \Phi_{1}^{\epsilon}$ and $B_{-\epsilon} \subset \Phi_{1}^{-\epsilon}$ which are invariant under $\tau_{\epsilon}$ and $\tau_{-\epsilon}$, respectively, and are both isotopic to $\Phi_{k}^{\epsilon}$. By Subsection 5.2.1 it follows that there exist $I$-pairs $\left(E_{\epsilon}, B_{\epsilon}\right) \subset\left(M_{\tilde{F}}^{\epsilon}, \tilde{F}\right)$ whose associated $\partial I$-subbundles are both isotopic to the subsurface $\Phi_{k}^{\epsilon}$ of $\tilde{F}$. After modifying these $I$-pairs by isotopies we obtain a semi-fibered submanifold $N=E_{+} \cup E_{-}$contained in $M$ whose semifiber $B$ is isotopic to the large subsurface $\Phi_{k}^{\epsilon}$ of $\tilde{F}$. We may take $N$ to be contained in int $M$.

We are now ready to show that if $\Phi_{k}^{\epsilon}$ is non-empty, then $\tilde{F}$ is a semi-fiber. First we argue that each component of $\partial N$ is a $\pi_{1}$-injective torus in $M$. Since $N$ has a large semi-fiber, it is clear that each component of $\partial N$ is $\pi_{1}$-injective in $N$. It therefore suffices to show that $N$ is $\pi_{1}$-injective in $M$. A homotopically non-trivial loop in $N$ which is contained in $B$ is homotopically non-trivial in $M$ because $B$ is $\pi_{1}$-injective in $\tilde{F}$. Now consider a loop $\alpha$ in $N$ which is not homotopic to a loop in $B$. After modifying $\alpha$ by a free homotopy we may take it to be a composition of paths $\alpha_{1}, \ldots, \alpha_{2 n}$ for some $n>0$ such that each $\alpha_{i}$ is a path in $E_{(-1)^{i}}$ which has its 
endpoints in $B$ and is not fixed-endpoint homotopic in $N$ to a path in $B$. The map of pairs $\alpha_{i}:(I, \partial I) \rightarrow\left(E_{(-1)^{i}}, B_{(-1)^{i}}\right)$ must be homotopic to a homeomorphism onto a fiber. Since the $I$-bundles $E_{+}$and $E_{-}$are essential in $M_{\tilde{F}}$ the path $\alpha_{i}$ is not fixed-endpoint homotopic in $M$ to a path in $\tilde{F}$. It follows that $N$ is $\pi_{1}$-injective in $M$.

Since $M$ is a simple knot manifold, every component of $\partial N$ is boundary parallel. Furthermore, since $N$ has a large semi-fiber, it cannot be homeomorphic to $S^{1} \times$ $S^{1} \times I$. Thus $C=\overline{M-N}$ is a collar on $\partial M$. The surface $A=\overline{\tilde{F}-B}$ is a $\pi_{1^{-}}$ injective subsurface of $\tilde{F}$ without disk component which is properly embedded in $C$. In particular $A$ is $\pi_{1}$-injective in $C$ and hence each component of $A$ is an annulus. Furthermore, by Wa, either $A$ has a boundary parallel component or $A$ is vertical in the sense that it is mapped by some homeomorphism of $C$ onto $S^{1} \times S^{1} \times I$ which maps $A$ to $X \times S^{1} \times I$, where $X$ is a finite subset of $S^{1}$. Assume that $A$ has a boundary parallel component $A_{0}$. Then $A_{0} \subset \tilde{F}$ is isotopic relative to $\partial A_{0}$ to an annulus $A_{0}^{\prime}$ in $\partial N$. But the torus $\partial N$ is a union of essential annuli which are components of the frontiers of the $I$-pairs $\left(E_{\epsilon}, B_{\epsilon}\right)$. Thus the inclusion map of the annulus $A_{0}^{\prime}$ can be regarded as a reduced homotopy between the inclusion maps of the two boundary components of $A_{0}$. In particular, $A_{0}^{\prime}$ is not homotopic into $\tilde{F}$. This is a contradiction, hence $A$ is vertical. It follows easily that the semi-fibration of $N$ can be extended over $C$ to obtain a semi-fibration of $M$ with semi-fiber $\tilde{F}$.

\subsection{Bounding the length of a reduced homotopy.}

Theorem 5.4.1. Let $F$ be a connected essential surface in a simple knot manifold $M$. Suppose that $F$ is not a semi-fiber. Let $g$ and $m$ denote respectively the genus and number of boundary components of $F$. Then any reduced homotopy in the pair $(M, F)$ having a large time-0 map has length at most $8 g+3 m-8$.

For the proof of this theorem we will need the following construction.

5.4.2. Suppose that $F$ is any connected, non-separating, essential surface in $M$, and let $\tilde{F}$ denote the boundary of a regular neighborhood $N$ of $F$. It is clear that $\tilde{F}$ has a unique transverse orientation such that $\tilde{F}$ is a splitting surface and $M_{(\tilde{F})}^{+}=N$. We shall call $\tilde{F}$, equipped with this transverse orientation, a splitting surface associated to $F$.

If $F$ is any connected, separating, essential surface in $M$, we refer to $F$ itself, equipped with either transverse orientation, as a splitting surface associated to $F$.

\subsection{3.}

Proof of Theorem 5.4.1. Let $\tilde{F}$ be a splitting surface associated to $F$. Since $F$ is not a semi-fiber, it is clear that $\tilde{F}$ is not a semi-fiber. We define the subsurfaces $\Phi_{k}^{\epsilon}$ for $k \geq 0$ and $\epsilon \in\{ \pm 1\}$ as in Subsection 5.2. Let $H$ be a reduced homotopy of length $l$ in the pair $(M, F)$ starting on the $\epsilon$ side. Let us set $\tilde{l}=l$ if $F$ separates $M$, and $\tilde{l}=2 l$ if $F$ does not separate $M$. Then the homotopy $H$ determines a reduced homotopy $\tilde{H}$ of length $\tilde{l}$ in the pair $(M, \tilde{F})$ such that $\tilde{H}_{0}$ is large. This is obvious if $F$ separates; if $F$ does not separate, it follows from the fact that the two components of $\tilde{F}$ cobound a product. Let $\tilde{g}$ and $\tilde{m}$ denote, respectively, the total genus and number of boundary components of $\tilde{F}$. We have $\tilde{g}=g$ and $\tilde{m}=m$ in the separating case, and $\tilde{g}=2 g$ and $\tilde{m}=2 m$ in the non-separating case. Hence 
it suffices to show that the length $\tilde{l}$ of $\tilde{H}$ is at most $8 \tilde{g}+3 \tilde{m}-8$ in the separating case, and is at most $8 \tilde{g}+3 \tilde{m}-16$ in the non-separating case.

Set $n=\left[\frac{\tilde{l}+1}{2}\right]$, and consider the subsurfaces

$$
\Phi_{1}^{\epsilon} \supset \Phi_{3}^{\epsilon} \supset \ldots \supset \Phi_{2 n-1}^{\epsilon} \supset \Phi_{2 n+1}^{\epsilon}
$$

of $\tilde{F}$. Since the reduced homotopy $\tilde{H}$ has length $\tilde{l} \geq 2 n-1$, it follows from Proposition 5.2.8 that $\Phi_{2 n-1}^{\epsilon} \neq \emptyset$. Since $\tilde{F}$ is not a semi-fiber, it now follows from Proposition 5.3.9 that $\Phi_{2 i-1}^{\epsilon}$ is not a regular neighborhood of $\Phi_{2 i+1}^{\epsilon}$ for $i=1, \ldots, n$. Furthermore, by Corollary 5.3.8, each of these surfaces has even Euler characteristic. Using these facts, we will show that $n \leq 4 \tilde{g}+3 \tilde{m} / 2-4$ in the separating case, and that $n \leq 4 \tilde{g}+3 \tilde{m} / 2-8$ in the non-separating case; this implies the desired conclusion, since $\tilde{l} \leq 2 n$.

If $A$ is any large subsurface of $\tilde{F}$, then we will set $c(A)=\operatorname{genus}(A)-3 \chi(A) / 2-$ $|A|$. Note that $c(A)$ is always non-negative, and is an integer if $A$ has even Euler characteristic. We have $c(\tilde{F})=4 \tilde{g}+3 \tilde{m} / 2-4$ in the separating case, and $c(\tilde{F})=4 \tilde{g}+$ $3 \tilde{m} / 2-8$ in the non-separating case. Hence it suffices to show that $n \leq c(\tilde{F})$. Thus the proof reduces to the following general claim: if $A$ and $B$ are large subsurfaces of $\tilde{F}$, each of which has even Euler characteristic, and if $B$ is contained in the interior of $A$, then $c(B)<c(A)$ unless $A$ is a regular neighborhood of $B$.

To prove the claim, it suffices to show that if $A_{0}$ is a component of $A$, and if we set $B_{0}=B \cap A_{0}$, then $c\left(B_{0}\right)<c\left(A_{0}\right)$ unless $A_{0}$ is a regular neighborhood of $B_{0}$. Note that we have genus $\left(B_{0}\right) \leq \operatorname{genus}\left(A_{0}\right)$ and, since $A$ and $B$ are large, $\chi\left(B_{0}\right) \geq \chi\left(A_{0}\right)$. Thus we need only consider the two cases where $B_{0}=\emptyset$ and where $B_{0}$ is connected. The case $B_{0}=\emptyset$ is easy because $c(A)>0$ for any nonempty large subsurface $A$. For the case where $B_{0}$ is connected we may assume that $\operatorname{genus}\left(B_{0}\right)=\operatorname{genus}\left(A_{0}\right)$ and $\chi\left(B_{0}\right)=\chi\left(A_{0}\right)$, and we must show that $A_{0}$ is a regular neighborhood of $B_{0}$. Since $A$ and $B$ are large, no component of $\overline{A_{0}-B_{0}}$ is a disk. Thus the condition $\chi\left(B_{0}\right)=\chi\left(A_{0}\right)$ implies that each component of $\overline{A_{0}-B_{0}}$ is an annulus. None of these annuli can separate $A_{0}$ since $B_{0}$ is connected. On the other hand, since $\operatorname{genus}\left(B_{0}\right)=\operatorname{genus}\left(A_{0}\right)$ there cannot exist a simple closed curve in $A_{0}$ which has non-zero intersection number with a core curve of an annulus component of $\overline{A_{0}-B_{0}}$. It follows that each component of $\overline{A_{0}-B_{0}}$ is a collar on a boundary component of $A_{0}$ and hence that $A_{0}$ is a regular neighborhood of $B_{0}$. This completes the proof of the claim, and of the theorem.

\section{BoundARY SLOPES OF ESSENTIAL SURFACES AND SINGULAR SURFACES}

Most of the work in this section is devoted to proving Theorem 6.2.1, which is a refinement of Theorem 5.4.1 and gives a bound on the length of a reduced homotopy whose time-0 map is an essential path. Combining Theorem 6.2.1 with the results in Section 3 we obtain a proof of Theorem 6.2.2 and its Corollaries 6.2.3, 6.2.4 and 6.2.5. These results give bounds on $\Delta(\alpha, \beta)$, where $\beta$ is a boundary slope and $\alpha$ is either another boundary slope, a very small filling slope or a Seifert-fibered filling slope.

We will need the following definition in this section.

Definition 6.0.1. Let $\tilde{F}$ be a splitting surface in a simple knot manifold $M$. We say that $\tilde{F}$ admits a long rectangle if there exists a reduced homotopy $H: I \times I \rightarrow$ 
$M$ in the pair $(M, \tilde{F})$ having length at least $|\partial \tilde{F}|$ in the pair $(M, \tilde{F})$ such that $H_{t}(\partial I) \subset \partial M$ for all $t \in I$ and $H_{0}$ is an essential path.

\subsection{Reduced homotopies and outer subsurfaces.}

6.1.1. Hypotheses. Throughout Subsection 6.1 we will assume that $M$ is a simple knot manifold and that $\tilde{F}$ is a splitting surface in $M$ which admits a long rectangle.

We will define $\Phi_{ \pm 1}$ and $\tau_{ \pm 1}$ as in Subsection 5.2. For every $k \geq 0$ we fix subsurfaces $\Phi_{k}^{ \pm 1}$ of $\tilde{F}$ for which the conclusions of Proposition 5.2.8 hold and homeomorphisms $h_{k}^{ \pm 1}$ for which the conclusions of Proposition 5.3.1 hold.

Lemma 6.1.2. For each $\epsilon \in\{ \pm 1\}$, every component of $\partial \tilde{F}$ is isotopic in $\tilde{F}$ to a unique boundary component of $\Phi_{1}^{\epsilon}$. Furthermore, if $c$ and $c^{\prime}$ are components of $\partial \tilde{F}$ which cobound an annulus component of $M_{\tilde{F}}^{\epsilon} \cap \partial M$, then the boundary components of $\Phi_{1}^{\epsilon}$ which are isotopic to $c$ and $c^{\prime}$ are interchanged by $\tau_{\epsilon}$.

Proof. Set $m=|\partial \tilde{F}|$. The existence of a long rectangle means that there is a reduced homotopy $H$ of length $m$, such that $H_{t}(\partial I) \subset \partial M$ for all $t \in I$ and $f=H_{0}$ is an essential path. As in Subsection 3.4 we extend $f$ to an admissible pair of glasses $\hat{f}: \Gamma \rightarrow \tilde{F}$, and extend $H$ to a length- $m$ reduced homotopy $\hat{H}$ with $\hat{H}_{0}=\hat{f}$. By construction the homotopy $\hat{H}$ has the property that for each of the "rims" $\bar{l}_{i}$, we have $\hat{H}_{t}\left(\bar{l}_{i}\right) \subset \partial M$ for all $t \in[0,1]$.

Write $\hat{H}$ as a composition of essential basic homotopies $\hat{H}^{1}, \ldots, \hat{H}^{m}$. Because the $\hat{H}^{i}$ are essential homotopies, $\hat{H}^{i}$ maps $\bar{l}_{1} \times I$ to an annulus component $A_{i}$ of $M_{\tilde{F}} \cap \partial M$ and maps the two components of $\bar{l}_{1} \times \partial I$ to distinct components of $\partial A_{i}$. Since $\hat{H}$ is reduced, the annuli $A_{i}$ and $A_{i+1}$ are on opposite sides of their common boundary component $\hat{H}_{1}^{i}\left(\bar{l}_{1}\right)=\hat{H}_{0}^{i+1}\left(\bar{l}_{1}\right)$ for each $i=1, \ldots, m-1$. Since $m$ is the number of components of $\tilde{F} \cap \partial M$, it follows that the annuli $A_{i}$ are distinct and that every annulus component of $M_{\tilde{F}} \cap \partial M$ appears as one of the $A_{i}$. If we set $A_{0}=A_{m}$, then for each component $c$ of $\partial \tilde{F}$ there is some $i$ with $0 \leq i<m$ such that $c$ is the common boundary curve of the two annuli $A_{i}$ and $A_{i+1}$, one of which is contained in $M_{\tilde{F}}^{+}$and the other in $M_{\tilde{F}}^{-}$.

To prove the first assertion of the lemma let $c$ be a component of $\partial \tilde{F}$ and let $\epsilon \in\{ \pm 1\}$ be given. The curve $c$ is a boundary component of some annulus $A_{j} \subset$ $M_{\tilde{F}}^{\epsilon} \cap \partial M$. Lemma 5.2.6 implies that $\hat{H}_{0}^{j}$ is homotopic to a map from $\Gamma$ to $\Phi_{1}^{\epsilon}$. In particular $c$ is homotopic in $\tilde{F}$ to a (singular) curve in $\Phi_{1}^{\epsilon}$. Since $c$ is a boundary component of $\tilde{F}$, it follows that $c$ is homotopic to a boundary component of $\Phi_{1}^{\epsilon}$.

To prove the second assertion, suppose that $c$ and $c^{\prime}$ are boundary curves of $\tilde{F}$ which cobound an annulus component $A$ of $M_{\tilde{F}}^{\epsilon} \cap \partial M$. Then we have $A=A_{j}$ for some $j$. Let $\gamma$ be the boundary component of $\Phi_{1}^{\epsilon}$ which is homotopic to $c$. Lemma 5.2.6 implies that $\hat{H}_{0}^{j}$ is homotopic to a map $g: \Gamma \rightarrow \Phi_{1}^{\epsilon}$ such that $g\left(\bar{l}_{1}\right)=\gamma$. Applying Lemma 5.2.7 to the homotopy $H^{j}$, with $g$ in place of $f$ and $\Gamma$ in place of $K$, we conclude that $c^{\prime}=\hat{H}_{1}^{j}\left(\bar{l}_{1}\right)$ is homotopic in $\tilde{F}$ to $\tau_{\epsilon}(\gamma)$. The assertion follows immediately.

Lemma 6.1.3. If $\epsilon \in\{ \pm 1\}$ and if $\gamma$ is a simple closed curve in $\Phi_{k}^{\epsilon}$ which is isotopic in $\tilde{F}$ to a component of $\partial \tilde{F}$, then $h_{k}^{\epsilon}(\gamma)$ is also isotopic to some component of $\partial \tilde{F}$.

Proof. For $k=0$ the assertion is trivial. To prove the lemma for $k=1$, we may assume that $\gamma$ is a component of $\partial \Phi_{k}^{\epsilon}$. Let $c$ denote the component of $\partial \tilde{F}$ which is 
isotopic in $\tilde{F}$ to $\gamma$, and let $A$ denote the annulus component of $M_{\tilde{F}}^{\epsilon} \cap \partial M$ having $c$ as a boundary curve. If $c^{\prime}$ denotes the other boundary curve of $A$, then it follows from Lemma 6.1.2 that $c^{\prime}$ is isotopic in $\tilde{F}$ to a boundary component $\gamma^{\prime}$ of $\Phi_{1}^{\epsilon}$ and that $h_{1}^{\epsilon}(\gamma)=\tau_{\epsilon}(\gamma)=\gamma^{\prime}$. This proves the assertion in this case.

Now assume that $k>1$ and that the assertion holds with $k$ replaced by $k-1$, both for $\epsilon=1$ and $\epsilon=-1$. Suppose we are given $\epsilon \in\{ \pm 1\}$ and a simple closed curve $\gamma \subset \Phi_{k}^{\epsilon} \subset \Phi_{k-1}^{\epsilon}$ which is isotopic in $\tilde{F}$ to a component of $\partial \tilde{F}$. We will apply Proposition 5.3.4 with $i=k-1$ and $j=1$. According to Proposition 5.3.4, the map $h_{k-1}^{\epsilon} \mid \Phi_{k}^{\epsilon}$ is homotopic to a map $g_{k-1}^{\epsilon}: \Phi_{k-1}^{\epsilon} \rightarrow \Phi_{1}^{-\epsilon}$ such that $h_{k}^{\epsilon}$ is homotopic to $h_{1}^{(-1)^{k-1} \epsilon} \circ g_{k-1}^{\epsilon}$. Now $g_{k-1}^{\epsilon}(\gamma)$ is homotopic to $h_{k-1}^{\epsilon}(\gamma)$ which, by the induction hypothesis, is homotopic to some component of $\partial \tilde{F}$. By the case $k=1$ of the proposition we know that $h_{1}^{(-1)^{k-1} \epsilon}\left(h_{k-1}^{\epsilon}(\gamma)\right)$ is homotopic to a component of $\partial \tilde{F}$. Since $h_{k}^{\epsilon}$ is homotopic to $h_{1}^{(-1)^{k-1} \epsilon} \circ g_{k-1}^{\epsilon}$ this shows that $h_{k}^{\epsilon}(\gamma)$ is homotopic to a component of $\partial \tilde{F}$.

Recall from Subsection 4.6 that if $A$ is a subsurface of a compact orientable surface $S$, then the outer part of $A$ is denoted $\dot{A}$.

Lemma 6.1.4. For either $\epsilon \in\{ \pm 1\}$ and for any large subsurface $A$ of $\Phi_{k}^{\epsilon}$, the outer part of $h_{k}^{\epsilon}(A)$ is $h_{k}^{\epsilon}(\dot{A})$.

Proof. Set $B=h_{k}^{\epsilon}(A)$. It follows from Lemma 6.1.3 that $h_{k}^{\epsilon}(\dot{A}) \subset \dot{B}$. Let $g: \Phi_{k}^{(-1)^{k+1} \epsilon} \rightarrow \Phi_{k}^{\epsilon}$ denote the inverse of $h_{k}^{\epsilon}$, so that $g(B)=A$. According to Proposition 5.3.3 the map $g$ is isotopic to $h_{k}^{(-1)^{k+1} \epsilon}$ as a map from $\Phi_{k}^{(-1)^{k+1} \epsilon}$ to $\Phi_{k}^{\epsilon}$. It therefore follows from Lemma 6.1.2 that $g(\dot{B}) \subset \dot{A}$, i.e. that $\dot{B} \subset h_{k}^{\epsilon}(\dot{A})$.

6.1.5. We now consider the outer parts of the surfaces $\Phi_{k}^{\epsilon}$ which, according to our conventions, are denoted $\dot{\Phi}_{k}^{\epsilon}$. Note that since

$$
\tilde{F}=\Phi_{0}^{\epsilon} \supset \Phi_{1}^{\epsilon} \supset \Phi_{2}^{\epsilon} \supset \cdots
$$

for $\epsilon \in\{ \pm 1\}$, it follows from Subsection 4.6 that

$$
\tilde{F}=\dot{\Phi}_{0}^{\epsilon} \supset \dot{\Phi}_{1}^{\epsilon} \supset \dot{\Phi}_{2}^{\epsilon} \supset \cdots .
$$

It follows from Lemma 6.1.4 that $h_{k}^{\epsilon}$ restricts to a homeomorphism from $\dot{\Phi}_{k}^{\epsilon}$ to $\dot{\Phi}_{k}^{(-1)^{k+1} \epsilon}$. This homeomorphism will be denoted by $\dot{h}_{k}^{\epsilon}$. In particular the involution $\tau_{\epsilon}=h_{1}^{\epsilon}$ of $\Phi_{1}^{\epsilon}$ restricts to an involution $\dot{\tau}_{\epsilon}=\dot{h}_{1}^{\epsilon}$ of $\dot{\Phi}_{1}^{\epsilon}$. It also follows from Lemma 6.1.4 that if $A$ is any large subsurface of $\Phi_{k}^{\epsilon}$, then $\dot{h}_{k}^{\epsilon}(\dot{A})$ is the outer part of $h_{k}^{\epsilon}(A)$. Note also that, according to Proposition 5.3.1, if $\tilde{F}$ is given a consistent orientation, $\dot{h}_{k}^{\epsilon}: \dot{\Phi}_{k}^{\epsilon} \rightarrow \tilde{F}$ is orientation reversing if $k$ is odd and orientation preserving if $k$ is even.

The following six results, 6.1.6-6.1.11, are analogues of 5.3.4-5.3.9. (Of course these results, unlike their counterparts in Section 4, depend on the hypothesis stated at the beginning of the section that $\tilde{F}$ admits a long rectangle.)

Proposition 6.1.6. Let $i$ and $j$ be non-negative integers, and set $k=i+j$. Then for each $\epsilon \in\{ \pm 1\}$, the map $\dot{h}_{i}^{\epsilon} \mid \dot{\Phi}_{k}^{\epsilon}$ is homotopic in $\tilde{F}$ to an embedding $\dot{g}_{i}^{\epsilon}: \dot{\Phi}_{k}^{\epsilon} \rightarrow$ $\dot{\Phi}_{j}^{(-1)^{i} \epsilon}$ such that $\dot{h}_{j}^{(-1)^{i} \epsilon} \circ \dot{g}_{i}^{\epsilon}$ is homotopic in $\tilde{F}$ to $\dot{h}_{k}^{\epsilon}$. 
Proof. By Proposition 5.3.4 we have that $h_{i}^{\epsilon} \mid \Phi_{k}^{\epsilon}$ is homotopic in $\tilde{F}$ to an embedding $g_{i}^{\epsilon}: \Phi_{k}^{\epsilon} \rightarrow \Phi_{j}^{(-1)^{i} \epsilon}$ such that $h_{j}^{(-1)^{i} \epsilon} \circ g_{i}^{\epsilon}$ is homotopic in $\tilde{F}$ to $h_{k}^{\epsilon}$. Set $\dot{g}_{i}^{\epsilon}=g_{i}^{\epsilon} \mid \dot{\Phi}_{k}^{\epsilon}$, and set $A=\dot{g}_{i}^{\epsilon}\left(\dot{\Phi}_{k}^{\epsilon}\right)$. To complete the proof it suffices to show that $A \subset \dot{\Phi}_{j}^{(-1)^{i} \epsilon}$.

Since $h_{j}^{(-1)^{i} \epsilon} \circ g_{i}^{\epsilon}$ is homotopic to $h_{k}^{\epsilon}$, the subsurface $h_{j}^{(-1)^{i} \epsilon}(A)$ is homotopic into $h_{k}^{\epsilon}\left(\dot{\Phi}_{k}^{\epsilon}\right)$ which, by Subsection 6.1 .5 , is equal to $\dot{\Phi}_{k}^{(-1)^{k+1} \epsilon}$. In particular $h_{j}^{(-1)^{i} \epsilon}(A)$ is homotopic into $\dot{\Phi}_{j}^{(-1)^{k+1} \epsilon}$. Since $h_{j}^{(-1)^{i} \epsilon}(A)$ is a large subsurface of $h_{j}^{(-1)^{i} \epsilon}\left(\Phi_{j}^{(-1)^{i} \epsilon}\right)=$ $\Phi_{j}^{(-1)^{k+1} \epsilon}$, and since $\dot{\Phi}_{j}^{(-1)^{k+1} \epsilon}$ is a union of components of $\Phi_{j}^{(-1)^{k+1} \epsilon}$, it follows that $h_{j}^{(-1)^{i} \epsilon}(A)$ is contained in $\dot{\Phi}_{j}^{(-1)^{k+1} \epsilon}$, which by Subsection 6.1 .5 is equal to $h_{j}^{(-1)^{i} \epsilon}\left(\dot{\Phi}_{j}^{(-1)^{i} \epsilon}\right)$. We therefore have $A \subset \dot{\Phi}_{j}^{(-1)^{i} \epsilon}$, as required.

Proposition 6.1.7. Let $i$ and $j$ be non-negative integers, and set $k=i+j$. Then for each $\epsilon \in\{ \pm 1\}$ the subsurface $\dot{h}_{i}^{\epsilon}\left(\dot{\Phi}_{k}^{\epsilon}\right)$ is isotopic in $\tilde{F}$ to $\dot{\Phi}_{i}^{(-1)^{i+1} \epsilon} \dot{\Lambda}_{\mathcal{L}} \dot{\Phi}_{j}^{(-1)^{i} \epsilon}$.

Proof. We may assume by Proposition 5.3.5 that $h_{i}^{\epsilon}\left(\Phi_{k}^{\epsilon}\right)=\Phi_{i}^{(-1)^{i+1} \epsilon} \wedge_{\mathcal{L}} \Phi_{j}^{(-1)^{i} \epsilon}$. It follows from Subsection 6.1.5 that $\dot{h}_{i}^{\epsilon}\left(\dot{\Phi}_{k}^{\epsilon}\right)$ is the outer part of $h_{i}^{\epsilon}\left(\Phi_{k}^{\epsilon}\right)$ and is therefore equal to the surface $\Phi_{i}^{(-1)^{i+1} \epsilon} \dot{\Lambda}_{\mathcal{L}} \Phi_{j}^{(-1)^{i} \epsilon}$. By Lemma 4.7 this surface is isotopic in $\tilde{F}$ to $\dot{\Phi}_{i}^{(-1)^{i+1} \epsilon} \dot{\wedge}_{\mathcal{L}} \dot{\Phi}_{j}^{(-1)^{i} \epsilon}$.

Lemma 6.1.8. For any non-negative integer $k$ and for each $\epsilon \in\{ \pm 1\}$ the subsurface $\dot{h}_{k}^{\epsilon}\left(\dot{\Phi}_{2 k+1}^{\epsilon}\right)$ is isotopic in $\tilde{F}$ to a subsurface $A$ of $\dot{\Phi}_{1}^{(-1)^{k} \epsilon}$ with the property that $\dot{\tau}_{(-1)^{k} \epsilon}(A)$ is a subsurface of $\dot{\Phi}_{1}^{(-1)^{k} \epsilon}$ which is isotopic to $A$ in $\dot{\Phi}_{1}^{(-1)^{k} \epsilon}$.

Proof. By Lemma 5.3.6 we know that $h_{k}^{\epsilon}\left(\Phi_{2 k+1}^{\epsilon}\right)$ is isotopic in $\tilde{F}$ to a subsurface $A_{0}$ of $\Phi_{1}^{(-1)^{k} \epsilon}$ with the property that $\tau_{(-1)^{k} \epsilon}\left(A_{0}\right)$ is isotopic to $A_{0}$ in $\Phi_{1}^{(-1)^{k} \epsilon}$. It follows from Subsection 6.1.5 that $\dot{h}_{k}^{\epsilon}\left(\dot{\Phi}_{2 k+1}^{\epsilon}\right)$ is isotopic in $\tilde{F}$ to $\dot{A}_{0}$. It also follows from Subsection 6.1.5 that $\dot{\tau}_{(-1)^{k} \epsilon}\left(\dot{A}_{0}\right)=h_{1}^{(-1)^{k} \epsilon}\left(\dot{A}_{0}\right)=\dot{h}_{1}^{(-1)^{k} \epsilon}\left(\dot{A}_{0}\right)$ is equal to the outer part of $h_{1}^{(-1)^{k} \epsilon}\left(A_{0}\right)=\dot{\tau}_{(-1)^{k} \epsilon}\left(A_{0}\right)$. Since $\dot{\tau}_{(-1)^{k} \epsilon}\left(A_{0}\right)$ is isotopic to $A_{0}$ it follows that $\dot{\tau}_{(-1)^{k} \epsilon}\left(\dot{A}_{0}\right)$ is isotopic in $\tilde{F}$ to $\dot{A}_{0}$. Since the subsurfaces $\dot{\tau}_{(-1)^{k} \epsilon}\left(\dot{A}_{0}\right)$ and $\dot{A}_{0}$ of $\dot{\Phi}_{1}^{\epsilon}$ are isotopic in $\tilde{F}$, it follows from Lemma 4.1 that they are isotopic in $\dot{\Phi}_{1}^{(-1)^{k} \epsilon}$. Thus we may take $A=\dot{A}_{0}$.

Proposition 6.1.9. For any non-negative integer $k$ and for each $\epsilon \in\{ \pm 1\}$ the surface $\dot{h}_{k}^{\epsilon}\left(\dot{\Phi}_{2 k+1}^{\epsilon}\right)$ is isotopic in $\tilde{F}$ to a subsurface of $\dot{\Phi}_{1}^{(-1)^{k} \epsilon}$ which is invariant under the free involution $\dot{\tau}_{(-1)^{k} \epsilon}$. In particular, $\dot{\Phi}_{2 k+1}^{\epsilon}$ admits a free involution.

Proof. This is an immediate consequence of Lemmas 6.1.8 and 4.5.

Corollary 6.1.10. For each $\epsilon \in\{ \pm 1\}$ and each odd integer $k>0$, the Euler characteristic $\chi\left(\dot{\Phi}_{k}^{\epsilon}\right)$ is even.

Proposition 6.1.11. Let $k$ be a non-negative integer and let $\epsilon \in\{ \pm 1\}$ be given. If $\dot{\Phi}_{k}^{\epsilon}$ and $\dot{\Phi}_{k+2}^{\epsilon}$ are isotopic in $\tilde{F}$, then either $\dot{\Phi}_{k}^{\epsilon}=\emptyset$ or $\tilde{F}$ is a semi-fiber.

Proof. This is formally identical with the proof of Proposition 5.3.9. All occurrences of $\Phi, h, \tau$ and $\wedge_{\mathcal{L}}$ are replaced by $\dot{\Phi}, \dot{h}, \dot{\tau}$ and $\dot{\Lambda}_{\mathcal{L}}$, respectively. References to 
Propositions 5.3.5 and 5.3.7 are replaced by references to Proposition 6.1.7 and 6.1 .9 .

6.2. The distance bounds. The next result is a strengthened version of Theorem 5.4.1 that applies to a reduced homotopy whose time- 0 map is an essential path.

Theorem 6.2.1. Let $F$ be a connected essential surface in a simple knot manifold $M$. Suppose that $F$ is not a semi-fiber. Set $g=\operatorname{genus}(F)$ and $m=|\partial F|$. Let $H$ be any reduced homotopy in the pair $(M, F)$ such that $H_{0}$ is an essential path in $F$ and $H_{t}(\partial I) \subset \partial M$ for each $t \in I$. Then the length of $H$ is at most $4 g+3 m-4$.

Proof. The proof will be similar to that of Theorem 5.4.1. Let $\tilde{F}$ be a splitting surface associated to $F$. Since $F$ is not a semi-fiber, it follows that $\tilde{F}$ is not a semi-fiber.

Let $l$ denote the length of $H$. We may assume $l>m$. Set $\tilde{l}=l$ if $F$ is separating and $\tilde{l}=2 l$ if $F$ is non-separating. As in the proof of Theorem 5.4.1, the homotopy $H$ determines a homotopy $\tilde{H}$ of length $\tilde{l}$ in the pair $(M, \tilde{F})$ such that $\tilde{H}_{0}$ is an essential path. Let $\tilde{g}$ and $\tilde{m}$ denote, respectively, the total genus and number of boundary components of $\tilde{F}$. We have $\tilde{m}=m$ and $\tilde{g}=g$ in the separating case and $\tilde{m}=2 m, \tilde{g}=2 g$ in the non-separating case. It therefore suffices to prove that $\tilde{l} \leq 4 \tilde{g}+3 \tilde{m}-4$ if $F$ is separating and $\tilde{l} \leq 4 \tilde{g}+3 \tilde{m}-8$ if $F$ is non-separating.

Since $l>m$ we also have $\tilde{l}>\tilde{m}$. In particular this means that $\tilde{F}$ admits a long rectangle and hence that the hypotheses stated in Subsection 6.1.1 hold. We define the subsurfaces $\Phi_{k}^{ \pm 1}$ and $\dot{\Phi}_{k}^{ \pm 1}$ for $k \geq 0$ as in Subsections 6.1.1 and 6.1.5.

Let us say that a subsurface $A$ of $\tilde{F}$ is allowable if $A$ is a large subsurface with even Euler characteristic and if $A$ is an outer subsurface (see Subsection 4.6). If $A$ is any large subsurface of $\tilde{F}$, let $\nu(A)$ denote the number of components of $\partial \tilde{F}$ which are homotopic into $A$. Note that $\nu(A)>0$ for any non-empty allowable subsurface A. We set

$$
c(A)=\operatorname{genus}(A)-\frac{\chi(A)}{2}-|A|+\nu(A)=2 \operatorname{genus}(A)+\frac{|\partial A|}{2}-2|A|+\nu(A) .
$$

Note that $c(A)$ is non-negative and integer-valued if $A$ is allowable. Moreover, $c(A)>0$ if $A$ is non-empty and allowable.

Set $n=\left[\frac{\tilde{l}+1}{2}\right]$ and define $\epsilon \in\{ \pm 1\}$ by the condition that the homotopy $\tilde{H}$ starts on the $\epsilon$-side. Consider the subsurfaces

$$
\dot{\Phi}_{1}^{\epsilon} \supset \dot{\Phi}_{3}^{\epsilon} \supset \ldots \supset \dot{\Phi}_{2 n-1}^{\epsilon} \supset \dot{\Phi}_{2 n+1}^{\epsilon} .
$$

Since the hypotheses stated in Subsection 6.1.1 hold, Corollary 6.1.10 implies that each of these surfaces has even Euler characteristic, and, in view of the definition of the $\dot{\Phi}_{k}^{\epsilon}$, it follows that each of these surfaces is allowable. On the other hand, since the reduced homotopy $\tilde{H}$ has length $\tilde{l} \geq 2 n-1$, it follows from Subsection 3.4 and Proposition 5.2.8 that there is an admissible pair of glasses $\gamma: \Gamma \rightarrow \tilde{F}$ which is homotopic in $\tilde{F}$ to a map from $\Gamma$ to $\Phi_{2 n-1}^{\epsilon}$. In particular there is a map $\alpha: S^{1} \rightarrow \partial \tilde{F}$ which is homotopic in $\tilde{F}$ to a map from $S^{1}$ to a component $A$ of $\Phi_{2 n-1}^{\epsilon}$. It follows that $A$ must be an outer component of $\Phi_{2 n-1}^{\epsilon}$, and hence that $\dot{\Phi}_{2 n-1}^{\epsilon} \neq \emptyset$. Since $\tilde{F}$ is not a semi-fiber and since the hypotheses stated in Subsection 6.1.1 hold, we may apply Proposition 6.1.11 to conclude that $\dot{\Phi}_{2 i-1}^{\epsilon}$ is not a regular neighborhood of $\dot{\Phi}_{2 i+1}^{\epsilon}$ for $i=1, \ldots, n$. We will show that in this situation $n \leq 2 \tilde{g}+3 \tilde{m} / 2-2$ if 
$F$ is separating and $n \leq 2 \tilde{g}+3 \tilde{m} / 2-4$ if $F$ is non-separating. Since $\tilde{l} \leq 2 n$ this will imply the theorem.

We have $c(\tilde{F})=2 \tilde{g}+3 \tilde{m} / 2-2$ if $F$ is separating and $c(\tilde{F})=2 \tilde{g}+3 \tilde{m} / 2-4$ if $F$ is non-separating. Hence it will suffice to show that if $A$ and $B$ are two allowable subsurfaces with $B \subset$ int $A$, then $c(B)<c(A)$ unless $A$ is a regular neighborhood of $B$.

As in the proof of Theorem 5.4.1 it suffices to show, for a component $A_{0}$ of $A$ and the subsurface $B_{0}=B \cap A_{0}$, that $c\left(B_{0}\right)<c\left(A_{0}\right)$ if $A_{0}$ is not a regular neighborhood of $B_{0}$. We have that genus $\left(B_{0}\right) \leq \operatorname{genus}\left(A_{0}\right)$, that $\chi\left(B_{0}\right) \geq \chi\left(A_{0}\right)$, and $\nu\left(B_{0}\right) \leq \nu\left(A_{0}\right)$. Thus we need only consider the two cases where $\left|B_{0}\right| \leq\left|A_{0}\right|=$ 1, i.e. where $B_{0}$ is empty and where $B_{0}$ is connected. The case $B_{0}=\emptyset$ is easy since $A_{0} \neq \emptyset$ implies $c\left(A_{0}\right)>0$. For the case where $B_{0}$ is connected we observe that if $c\left(B_{0}\right)=c\left(A_{0}\right)$, then we have genus $\left(B_{0}\right)=\operatorname{genus}\left(A_{0}\right)$ and $\chi\left(B_{0}\right)=\chi\left(A_{0}\right)$; it then follows as in the proof of Theorem 5.4.1 that $A_{0}$ is a regular neighborhood of $B_{0}$.

We are now ready to state and prove one of the main results of this paper. Recall that the function $N(s, n, v)$ was defined in Subsection 3.7.

Theorem 6.2.2. Let $M$ be a simple knot manifold, and let $F \subset M$ be an essential bounded surface with boundary slope $\beta$ which is not a semi-fiber. Let $(S, X, h)$ be a singular surface which is well positioned with respect to $F$ and has boundary slope $\alpha$. Set $s=$ genus $S, n=|\partial S-X|, v=|X|, g=$ genus $F$ and $m=|\partial F|$. Then

$$
\Delta(\alpha, \beta) \leq\left(\frac{4 g-3}{m}+3\right) N(s, n, v) .
$$

Proof. According to Proposition 3.9, there exists an essential homotopy $H: I \times I \rightarrow$ $M$ having length

$$
l \geq \frac{m \Delta(\alpha, \beta)}{N(s, n, v)}-1
$$

such that $H_{0}$ is an essential path in $F$ and $H_{t}(\partial I) \subset \partial M$ for all $t \in I$. By Theorem 6.2.1 we have that

$$
l \leq 4 g+3 m-4 .
$$

The conclusion follows from the inequalities (1) and (2).

Corollary 6.2.3. Let $M$ be a simple knot manifold, and let $F \subset M$ be an essential bounded surface with boundary slope $\beta$ which is not a semi-fiber. Set $g=$ genus $F$ and $m=|\partial F|$. Let $\alpha$ be a slope in $\partial M$. If $M(\alpha)$ is very small, or more generally if $F \subset M \subset M(\alpha)$ is not $\pi_{1}$-injective in $M(\alpha)$, then

$$
\Delta(\alpha, \beta) \leq \frac{20 g-15}{m}+15 .
$$

Proof. We invoke Corollary 2.3 to obtain a singular surface $(S, X, h)$, well positioned with respect to $F$, such that genus $S=0$ and $|X|=1$. The conclusion now follows from Theorem 6.2 .2 because for any $v \geq 1$ we have $N(0,1, v) \leq 5$.

Corollary 6.2.4. Let $M$ be a simple knot manifold, and let $F \subset M$ be an essential bounded surface with boundary slope $\beta$ which is not a semi-fiber. Let $\alpha$ be a slope 
in $\partial M$. Set $g=$ genus $F$ and $m=|\partial F|$. If $M(\alpha)$ is a Seifert fibered space or if there exists a $\pi_{1}$-injective map from $S^{1} \times S^{1}$ to $M$, then

$$
\Delta(\alpha, \beta) \leq \frac{24 g-18}{m}+18 .
$$

Proof. We invoke Corollary 2.6 to obtain a singular surface $(S, X, h)$, well positioned with respect to $F$, such that either genus $S=0$ and $|X|=1$, or genus $S=1$ and $|X|=0$. The conclusion now follows from Theorem 6.2 .2 because for any $v \geq 1$ we have $N(0,1, v) \leq 5$ and $N(1,0, v)=6$.

Corollary 6.2.5. Let $M$ be a simple knot manifold. Suppose, for $i=1,2$, that $F_{i} \subset M$ is an essential bounded surface of genus $g_{i}$ with boundary slope $\beta_{i}$. Let $m_{i}=\left|\partial F_{i}\right|$. If $F_{1}$ is not a semi-fiber, then we have

$$
\Delta\left(\beta_{1}, \beta_{2}\right) \leq\left(\frac{4 g_{1}-3}{m_{1}}+3\right)\left(\left[\frac{12 g_{2}-12}{m_{2}}\right]+6\right) .
$$

Proof. We apply Proposition 2.1 with $F=F_{1}$ and $S=F_{2}$. This gives a singular surface $\left(F_{2}, \partial F_{2}, h\right)$ which is well positioned with respect to $F_{1}$. Theorem 6.2.2 then implies that

$$
\Delta\left(\beta_{1}, \beta_{2}\right) \leq\left(\frac{4 g_{1}-3}{m_{1}}+3\right) N\left(g_{2}, 0, m_{2}\right) .
$$

Note that since $M$ is a simple knot manifold, the surface $F_{2}$ cannot be a disk or an annulus. It then follows from Subsection 3.7 that

$$
N(s, n, v)=\left[\frac{12 g_{2}-12}{m_{2}}\right]+6 .
$$

Ian Agol has informed us that a slightly stronger estimate follows from the techniques in his paper $\mathrm{A}$. By combining his Theorem 5.1 with the proof of his Theorem 8.1 he can show under the hypotheses of Corollary 6.2.5 that

$$
\Delta\left(\beta_{1}, \beta_{2}\right) \leq \frac{36}{3.35}\left(\frac{2 g_{1}-2}{m_{1}}+1\right)\left(\frac{2 g_{2}-2}{m_{2}}+1\right) .
$$

In particular the coefficient of $g_{1} g_{2} / m_{1} m_{1}$ is less than 43 for this estimate, while in the estimate provided by Corollary 6.2.5 the corresponding coefficient is 48. Agol's methods depend on the rigorous computational results of Cao and Meyerhoff [CM].

Corollary 6.2.6. Let $M$ be a simple knot manifold, and let $F \subset M$ be an essential bounded surface with boundary slope $\beta$ which is not a semi-fiber. Set $g=$ genus $F$ and $m=|\partial F|$. If $\alpha$ is the boundary slope of an essential planar surface in $M$, then

$$
\Delta(\alpha, \beta) \leq \frac{20 g-15}{m}+15 .
$$

Proof. If $M(\alpha)$ is reducible, then there is an essential planar surface $F_{2}$ with boundary slope $\alpha$. The result follows from Corollary 6.2 .5 by taking $F=F_{1}, \beta=\beta_{1}$, and $\alpha=\beta_{2}$.

Still another corollary to Theorem 6.2.2 can be obtained by using Corollary 2.5. The reader is invited to formulate the statement. 


\section{Tight SURfaCES}

The goal of this section is to prove Theorem 7.4.2, which provides a major improvement on conclusion of Theorem 6.2.1 in the special case where $F$ is planar, i.e. $g=0$. This leads to corresponding improvements to the corollaries of the previous section in cases where the planarity assumption hold.

The techniques used in this section make use of some variants of the surfaces $\dot{\Phi}_{k}^{\epsilon}$ which are denoted $\breve{\Phi}_{k}^{\epsilon}$. We start with some preliminaries which are needed for the definition of these surfaces.

7.1. Perfect surfaces. In this subsection $S$ will denote a compact orientable surface of negative Euler characteristic. If $A$ is a subsurface of $S$ we will denote the frontier of $A$ by $\operatorname{Fr} A$.

Definition 7.1.1. A subsurface $A$ of $S$ will be said to be perfect if

(i) $A$ is $\pi_{1}$-injective;

(ii) $A$ contains $\partial S$; and

(iii) every component of $A$ contains a component of $\partial S$.

Thus if $A$ is a perfect subsurface of $S$ then each component of $A$ is either a large subsurface of $S$ which contains a component of $\partial S$ or a regular neighborhood of a component of $\partial S$.

Suppose that $A$ is a large outer subsurface of a compact orientable surface $S$. Let $A^{\prime}$ denote a subsurface in the non-ambient isotopy class of $A$ such that for every component $C$ of $\partial A^{\prime}$, either $C \subset \partial S$, or $C \subset$ int $S$ and $C$ is not parallel to any component of $\partial S$. The surface $A^{\prime}$ is unique up to ambient isotopy. We define a perfection of $A$, denoted $\mathcal{P}(A)$, to be a surface of the form $A^{\prime} \cup N$, where $N$ is a regular neighborhood of the union of all components of $\partial S$ which are not contained in $\partial A^{\prime}$, and $N \cap A^{\prime}=\emptyset$. Note that $\mathcal{P}(A)$ is a perfect surface and that the ambient isotopy class of $\mathcal{P}(A)$ is uniquely determined by the non-ambient isotopy class of $A$. Moreover, if $c$ is a component of $\partial S$ and if $C$ is the component containing $c$, then no other component of $\mathcal{P}(A)$ contains a curve isotopic to $c$.

7.1.2. There is an obvious inverse operation to perfection: if $B$ is a perfect subsurface of $S$, then $\mathcal{L}(B)$ is a large outer subsurface of $S$. It is clear that if $A$ is any large outer subsurface of $S$, then $\mathcal{L}(\mathcal{P}(A))$ is (non-ambiently) isotopic to $A$, and that if $B$ is any perfect subsurface of $S$, then $B$ is ambiently isotopic to $\mathcal{P}(\mathcal{L}(B)$ ). Thus we have a natural bijective correspondence between non-ambient isotopy classes of large outer subsurfaces of $S$ and ambient isotopy classes of perfect subsurfaces of $S$.

The following lemma will be needed in Subsection 7.2.

Lemma 7.1.3. Let $P$ be a perfect subsurface of $S$ and suppose that $\tau$ is a free involution of $P$. Suppose that $A \subset P$ is a large outer subsurface of $S$ which is invariant under $\tau$. Then $A$ has a perfection which is contained in $P$ and invariant under $\tau$.

Proof. By replacing $A$ with a slightly smaller $\tau$-invariant surface we may assume that $A$ is contained in int $S$. Let $A^{\prime}$ be the union of $A$ with all of the annuli in $S$ which have one boundary component in $\partial A$ and one boundary component in $\partial S$. Since $P$ is perfect, $A$ is contained in $P$ and invariant under $\tau$. Thus $\partial S-\partial A^{\prime}$ is also invariant under $\tau$. We define the required perfection of $A$ to be the union of $A^{\prime}$ with a suitably small $\tau$-invariant regular neighborhood of $\partial S-\partial A^{\prime}$. 
7.1.4. If $A$ and $B$ are two perfect subsurfaces of $S$, then we define the perfect intersection of $A$ and $B$, denoted $A \wedge_{\mathcal{P}} B$, to be $\mathcal{P}\left(\mathcal{L}(A) \wedge_{\mathcal{L}} \mathcal{L}(B)\right)$. Observe that a perfect subsurface of $S$ is homotopic into $A \wedge_{\mathcal{P}} B$ if and only if it is homotopic into both $A$ and $B$. It follows from this together with Lemma 4.1, that $A \wedge_{\mathcal{P}} B$ is isotopic to $B \wedge_{\mathcal{P}} A$ and that $\left(A \wedge_{\mathcal{P}} B\right) \wedge_{\mathcal{P}} C$ is isotopic to $A \wedge_{\mathcal{P}}\left(B \wedge_{\mathcal{P}} C\right)$.

Now let $A$ and $B$ be large outer subsurfaces of $S$. It follows from the definition of perfect intersection and the bijective correspondence described in Definition 7.1.1 that

$$
\mathcal{P}(A) \wedge_{\mathcal{P}} \mathcal{P}(B)=\mathcal{P}\left(A \wedge_{\mathcal{L}}^{\cdot} B\right) .
$$

Proposition 7.1.5. Let $P$ and $Q$ be perfect surfaces of $S$. Then there exist (perfect) surfaces $P_{1}$ and $Q_{1}$ which are ambiently isotopic to $P$ and $Q$ respectively, such that the frontiers of $P_{1}$ and $Q_{1}$ meet transversely and $P \wedge_{\mathcal{P}} Q$ is ambiently isotopic to the union of all components of $P_{1} \cap Q_{1}$ which meet $\partial S$.

Proof. Set $A=\mathcal{L}(P)$ and $B=\mathcal{L}(Q)$. According to Proposition 4.2, $A$ and $B$ are non-ambiently isotopic to surfaces $A_{0} \subset$ int $S$ and $B_{0} \subset$ int $S$ such that $\partial A_{0}$ and $\partial B_{0}$ meet transversely and $\mathcal{L}\left(A_{0} \cap B_{0}\right)$ is isotopic to $A \wedge_{\mathcal{L}} B$. Let $A_{1}$ be the union of $A_{0}$ with all of the annular components of $\overline{S-A_{0}}$ which meet $\partial S$. It is clear that $A_{1}$ is ambiently isotopic to $A$. In the same way, using $B_{0}$, we define a subsurface $B_{1}$ which is ambiently isotopic to $B$. We claim that $\mathcal{L}\left(A_{1} \cap B_{1}\right)$ is isotopic to $A \wedge_{\mathcal{L}} B$. According to Proposition 4.2 and Definition 4.3 it suffices to show that property (*) of Proposition 4.2 holds with $C$ replaced by $\mathcal{L}\left(A_{1} \cap B_{1}\right)$. The "only if" part of $(*)$ is clear because $A_{1}$ is isotopic to $A$ and $B_{1}$ is isotopic to $B$. Since the "if" part of Proposition 4.2 holds with $C$ replaced by $\mathcal{L}\left(A_{0} \cap B_{0}\right)$, the claim will follow once we show that $\mathcal{L}\left(A_{0} \cap B_{0}\right) \subset \mathcal{L}\left(A_{1} \cap B_{1}\right)$. Since $A_{0} \cap B_{0} \subset A_{1} \cap B_{1}$, it suffices to show that $A_{1} \cap B_{1}$ is $\pi_{1}$-injective. But since $\chi(S)<0$, any homotopically trivial simple closed curve $\gamma \subset A_{1} \cap B_{1}$ bounds a unique disk $D$ in $S$; since $A_{1}$ and $B_{1}$ are $\pi_{1}$-injective we must have $D \subset A_{1}$ and $D \subset B_{1}$ and hence $D \subset A_{1} \cap B_{1}$. This proves the claim.

Thus we may take $A \wedge_{\mathcal{L}} B$ to be equal to $\mathcal{L}\left(A_{1} \cap B_{1}\right)$. Let $W$ denote the union of the large components of $A_{1} \cap B_{1}$ that meet $\partial S$. We next assert that $W$ is $A \wedge_{\mathcal{L}} B$. To prove this it suffices to show that every large outer component $X$ of $A_{1} \cap B_{1}$ contains a component of $\partial S$. By the definition of an outer component (see Subsection 4.6), $X$ contains a closed curve $\gamma \subset$ int $S$ which is the frontier of an annulus $\alpha \subset S$. Since $\gamma \subset A_{1}$ and $\gamma \subset B_{1}$, it follows from the construction of $A_{1}$ and $B_{1}$ that $\alpha$ is contained in both $A_{1}$ and $B_{1}$, and hence in $X$. In particular, $X$ contains a component of $\partial S$ as required.

Now let $N$ be regular neighborhood of $\partial S$. We may assume $N$ to be chosen so that each component of $N$ is disjoint from the frontiers of $A_{1}$ and $B_{1}$. We set $P_{1}=A_{1} \cup N$ and $Q_{1}=B_{1} \cup N$. It is clear that $P_{1}$ and $Q_{1}$ are isotopic to $P$ and $Q$, respectively. It is also clear that the union $Z$ of the components of $P_{1} \cap Q_{1}$ which meet $\partial S$ is equal to $W \cup N$. Since $W$ is $A \wedge_{\mathcal{L}} B$, we have that $Z=\mathcal{P}(W)=\mathcal{P}\left(A \wedge_{\mathcal{L}}^{\dot{L}} B\right)$. But by definition we have that $P \wedge_{\mathcal{P}} Q=\mathcal{P}\left(A \wedge_{\mathcal{L}}^{\dot{L}} B\right)$. This completes the proof.

7.1.6. Suppose that $P$ and $Q$ are perfect subsurfaces of $S$. Let $C$ be a component of the perfect intersection $P \wedge_{\mathcal{P}} Q$. Since $C$ is isotopic to a subsurface of $P$, there must be a component $P_{0}$ of $P$ such that $C$ is isotopic to a subsurface of $P_{0}$. We will say in this situation that $C$ is isotopically contained in $P_{0}$. Let $c$ be a component of 
$\partial S$ which is contained in $C$. Then, since there is a unique component of $P$ which contains a curve isotopic to $c$, it follows that $P_{0}$ is the unique component of $P$ which contains a surface isotopic to $C$. Thus each component of $P \wedge_{\mathcal{P}} Q$ is isotopically contained in a unique component of $P$.

Definition 7.1.7. Let $A \subset S$ be a perfect surface. A component $A_{0}$ of $A$ said to be tight if $A_{0}$ is planar and the frontier of $A_{0}$ in $S$ is a simple closed curve. We define the size of a tight component $A_{0}$ of $A$ to be the number of components of $\partial S$ which are contained in $A_{0}$. We will denote the size of $A_{0}$ by $s\left(A_{0}\right)$.

Proposition 7.1.8. Let $P$ and $Q$ be perfect subsurfaces of $S$ and let $P_{0}$ be a tight component of $P$. Assume that every tight component of $Q$ has size at least $s\left(P_{0}\right)$. Then every component of $P \wedge_{\mathcal{P}} Q$ which is isotopically contained in $P_{0}$ is tight and has size at most $s\left(P_{0}\right)$. Furthermore if $P_{0}$ contains only one component of $P \wedge_{\mathcal{P}} Q$ then this component is isotopic to $P_{0}$.

Proof. By Proposition 7.1.5 we may assume that $P$ and $Q$ have been chosen within their isotopy classes so that the frontiers of $P$ and $Q$ meet transversely and $Z=$ $P \wedge_{\mathcal{P}} Q$ is the union of all components of $P \cap Q$ which meet $\partial S$.

Consider first the case in which $P_{0}$ contains at least one component of $\operatorname{Fr} Q$. Note that since $Q$ is perfect, the components of $\operatorname{Fr} Q$ are homotopically non-trivial simple closed curves in $S$. Since $P_{0}$ is planar and has connected frontier, every homotopically non-trivial simple closed curve $\gamma$ in int $P_{0}$ is the frontier in $S$ of a unique subsurface $W_{\gamma}$ of $P_{0}$; the non-triviality of $\gamma$ implies that $W_{\gamma}$ is not a disk and hence that $W_{\gamma} \cap \partial S \neq \emptyset$. Among all components of $\operatorname{Fr} Q$ contained in $P_{0}$ we choose one, $\gamma_{0}$, such that $W_{\gamma_{0}}$ is minimal with respect to inclusion. The minimality implies that $W_{\gamma_{0}}$ is either a component of $Q$ or of $\overline{S-Q}$. But since $Q$ is perfect, we have $\partial S \subset Q$ and hence $W_{\gamma_{0}} \cap Q \supset W_{\gamma_{0}} \cap \partial S \neq \emptyset$. Hence $W_{\gamma_{0}}$ must be a component of $Q$. Since $W_{\gamma_{0}}$ is contained in the planar surface $P_{0}$ and has connected frontier, it is in fact a tight component of $Q$ with $s\left(W_{\gamma_{0}}\right) \leq s\left(P_{0}\right)$. On the other hand the hypothesis of the proposition implies that $s\left(W_{\gamma_{0}}\right) \geq s\left(P_{0}\right)$. It now follows that $P_{0}$ is a regular neighborhood of $W_{\gamma_{0}}$. Clearly $W_{\gamma_{0}}$ is a component of $P \cap Q$ and hence of $Z$. Since the annulus $\overline{P_{0}-W_{\gamma_{0}}}$ is disjoint from $\partial S$, it follows that $W_{\gamma_{0}}$ is the only component of $P_{0} \cap Q$ which meets $\partial S$. Hence $W_{\gamma_{0}}$ is the only component of $Z$ which is contained in $P_{0}$. Since $W_{\gamma_{0}}$ is isotopic to its regular neighborhood $P_{0}$, both conclusions of the proposition are established in this case.

There remains the case in which each component of $P_{0} \cap \operatorname{Fr} Q$ is a properly embedded arc in $P_{0}$ having both endpoints on $F r P_{0}$. It follows that any component of $P_{0} \cap Q$ is a planar surface whose frontier in $S$ is a simple closed curve. In particular, if $C$ is a component of $P_{0} \cap Q$ which contains a component of $\partial S$, then $C$ is a tight component of $Z$. It is also clear that $s(C) \leq s\left(P_{0}\right)$. To prove the last assertion of the proposition in this case, assume that $C$ is the only tight component of $Z$ which is contained in $P_{0}$. Then the frontier in $P_{0}$ of $C$ is a collection of properly embedded arcs which are parallel to subarcs of $\mathrm{Fr} P_{0}$. It then follows that $C$ is isotopic to $P_{0}$.

Proposition 7.1.9. Suppose that $S$ is planar and let $P \neq S$ be a perfect subsurface of $S$. Then $P$ has a tight component.

Proof. Since $P \neq S$ we have $\operatorname{Fr} P \neq \emptyset$. Since $P$ is perfect, each component of $\operatorname{Fr} P$ is a homotopically non-trivial curve in $S$ and hence is the frontier of two planar 
subsurfaces of $S$, neither of which is a disk. Among all subsurfaces $A$ of $S$ such that $\operatorname{Fr} A$ consists of a single component of $\operatorname{Fr} S$ we choose one, say $A_{0}$, which is minimal with respect to inclusion. Since $A_{0}$ is not a disk, we have $A_{0} \cap \partial S \neq \emptyset$. The minimality implies that either $A_{0}$ is a component of $P$ or of $\overline{S-P}$. But since $P$ is perfect, we have $\partial S \subset P$ and hence $A_{0} \cap P \supset A_{0} \cap \partial S \neq \emptyset$. Thus $A_{0}$ is a component of $P$ and by definition is tight.

We record here a simple lemma that will be needed in the next subsection.

Lemma 7.1.10. Let $A$ be an oriented annulus, and let $\alpha$ be a component of $\partial A$. Let $f$ and $g$ be two embeddings of $A$ into an orientable surface $F$. Suppose that $f(\alpha)=g(\alpha)=c$, where $c$ is a component of $\partial F$, and that $f$ and $g$ carry the orientation of $A$ to the same orientation of $F$. Then $f$ and $g$ are homotopic.

\subsection{Reduced homotopies and perfect subsurfaces.}

7.2.1. Throughout Subsection 7.2 we will assume that $M$ is a simple knot manifold and that $\tilde{F}$ is a splitting surface in $M$ which admits a long rectangle. Since this is the same assumption that was made in Proposition 6.1.1, the results of Subsection 6.1 may be applied in this subsection.

We will fix $\dot{\Phi}_{k}^{ \pm 1}, \dot{h}_{k}^{ \pm 1}$ and $\dot{\tau}_{ \pm 1}$ as in Subsection 6.1.5. Recall that the surfaces $\dot{\Phi}_{k}^{\epsilon}$ are only defined up to non-ambient isotopy. Here we suppose each surface $\dot{\Phi}_{k}^{\epsilon}$ to have been normalized within its non-ambient isotopy class so that if $C$ is a boundary component of $\dot{\Phi}_{k}^{\epsilon}$, then either $C \subset \partial \tilde{F}$, or $C \subset$ int $\tilde{F}$ and $C$ is not parallel to any component of $\partial \tilde{F}$. (It is clear that the surfaces $\dot{\Phi}_{k}^{\epsilon}$ can be chosen to have this property in addition to having the nestedness property stated in Subsection 6.1.5.)

By definition $\dot{\Phi}_{k}^{\epsilon}$ is a large outer subsurface for each $\epsilon \in\{ \pm 1\}$ and $k \geq 0$. Using the notation of Definition 7.1.1 we set

$$
\breve{\Phi}_{k}^{\epsilon}=\mathcal{P}\left(\dot{\Phi}_{k}^{\epsilon}\right) .
$$

Because of the way that the surfaces $\dot{\Phi}_{k}^{\epsilon}$ have been normalized, $\mathcal{P}\left(\dot{\Phi}_{k}^{\epsilon}\right)$ is the disjoint union of $\dot{\Phi}_{k}^{\epsilon}$ with $\mathcal{A}_{k}^{\epsilon}$, where $\mathcal{A}_{k}^{\epsilon}$ is a regular neighborhood of the union of all components of $\partial \tilde{F}$ which are not contained in $\dot{\Phi}_{k}^{ \pm 1}$.

We may assume that the regular neighborhoods $\mathcal{A}_{k}^{\epsilon}$ have been chosen so that $\breve{\Phi}_{k}^{\epsilon} \supset \mathcal{A}_{k+1}^{\epsilon}$. This means that for each $\epsilon \in\{ \pm 1\}$ we have

$$
\tilde{F}=\breve{\Phi}_{0}^{\epsilon} \supset \breve{\Phi}_{1}^{\epsilon} \supset \breve{\Phi}_{2}^{\epsilon} \supset \cdots .
$$

Note also that by Subsection 7.1.2 we have $\mathcal{L}\left(\breve{\Phi}_{k}^{\epsilon}\right)=\dot{\Phi}_{k}^{\epsilon}$ for each $\epsilon \in\{ \pm 1\}$ and each $k \geq 0$.

We denote by $m$ the number of boundary components of $\tilde{F}$. Since $\tilde{F}$ is a splitting surface the integer $m$ is even. We index the components of $\partial \tilde{F}$ as $c_{t}$, where $t$ ranges over $\mathbb{Z} / m \mathbb{Z}$. If $q$ is an integer we will denote the image of $q$ in $\mathbb{Z} / m \mathbb{Z}$ by $\bar{q}$.

We assume that the indexing of components of $\partial \tilde{F}$ has been done in such a way that for each $t \in \mathbb{Z} / m \mathbb{Z}$ the curves $c_{t}$ and $c_{t+1}$ cobound an annulus $R_{t} \subset \partial M$ whose interior is disjoint from $\partial \tilde{F}$. We may assume further that the indexing is done in such a way that $R_{\bar{q}} \subset M_{\tilde{F}}^{+}$for every even integer $q$ and $R_{\bar{q}} \subset M_{\tilde{F}}^{-}$for every odd integer $q$. 
7.2.2. For every integer $k \geq 0$ and $\epsilon \in\{ \pm 1\}$ we define a permutation $\sigma_{k}^{\epsilon}$ of $\mathbb{Z} / m \mathbb{Z}$ by

$$
\sigma_{k}^{\epsilon}(\bar{q})=\overline{q+(-1)^{q} \epsilon k}
$$

(Since $m$ is even, the coefficient $(-1)^{q}$ is determined by the congruence class $\bar{q}$.)

We observe that if $i$ and $j$ are non-negative integers with $i+j=k$, then for each $\epsilon \in\{ \pm 1\}$ we have

$$
\sigma_{k}^{\epsilon}=\sigma_{j}^{(-1)^{i} \epsilon} \circ \sigma_{i}^{\epsilon}
$$

We also observe that for each $k \geq 0$ and each $\epsilon \in\{ \pm 1\}$ we have

$$
\left(\sigma_{k}^{\epsilon}\right)^{-1}=\sigma_{k}^{(-1)^{k} \epsilon}
$$

From (1) and (2) it follows that for each $k \geq 0$ and each $\epsilon \in\{ \pm 1\}$ we have

$$
\sigma_{2 k+1}^{\epsilon}=\left(\sigma_{k}^{\epsilon}\right)^{-1} \circ \sigma_{1}^{(-1)^{k} \epsilon} \circ \sigma_{k}^{\epsilon}
$$

It is clear from (7.2.2.3) that $\sigma_{k}^{\epsilon}$ is a free involution if $k$ is odd.

Lemma 7.2.3. Let $k$ be a positive integer and fix $\epsilon \in\{ \pm 1\}$. If a component $c_{t}$ of $\partial \tilde{F}$ is contained in $\dot{\Phi}_{k}^{\epsilon}$ for some $t \in \mathbb{Z} / m \mathbb{Z}$, then $\dot{h}_{k}^{\epsilon}\left(c_{t}\right)=c_{\sigma_{k}^{\epsilon}(t)}$.

Proof. The lemma is trivial in the case $k=0$.

Consider the case $k=1$. By Lemma 6.1.2, together with our normalization of $\dot{\Phi}_{1}^{\epsilon}$, we know that every component of $\partial \tilde{F}$ is contained in $\dot{\Phi}_{1}^{\epsilon}$ and that $h_{1}^{\epsilon}=\tau_{\epsilon}$ interchanges two components $c$ and $c^{\prime}$ of $\partial \tilde{F}$ if and only if $c$ and $c^{\prime}$ cobound an annulus component of $M_{\tilde{F}}^{\epsilon} \cap M$. The definition of $\sigma_{1}^{\epsilon}$ thus implies that for every $t \in \mathbb{Z} / m \mathbb{Z}$ we have $h_{1}^{\epsilon}\left(c_{t}\right)=\tau_{\epsilon}\left(c_{t}\right)=c_{\sigma_{1}^{\epsilon}(t)}$.

Now, arguing inductively, we assume that $k>1$, that $c_{t}$ is a component of $\partial \tilde{F}$ contained in $\dot{\Phi}_{k}^{\epsilon} \subset \dot{\Phi}_{k-1}^{\epsilon}$ and that $\dot{h}_{k-1}^{\epsilon}\left(c_{t}\right)=c_{\sigma_{k-1}^{\epsilon}(t)}$. Applying Proposition 6.1.6 with $i=k-1$ and $j=1$ we see $\dot{h}_{k-1}^{\epsilon} \mid \dot{\Phi}_{k}^{\epsilon}$ is homotopic in $\tilde{F}$ to an embedding $\dot{g}_{k-1}^{\epsilon}: \dot{\Phi}_{k}^{\epsilon} \rightarrow \dot{\Phi}_{1}^{(-1)^{k-1} \epsilon}$ such that $\dot{h}_{k}^{\epsilon}$ is homotopic to $\dot{h}_{1}^{(-1)^{k-1} \epsilon} \circ \dot{g}_{k-1}^{\epsilon}$. Since $\dot{h}_{k-1}^{\epsilon}\left(c_{t}\right)=c_{\sigma_{k-1}^{\epsilon}(t)}$, the curve $\dot{g}_{k-1}^{\epsilon}\left(c_{t}\right) \subset \dot{\Phi}_{1}^{(-1)^{k-1} \epsilon}$ is homotopic in $\tilde{F}$ to the boundary component $c_{\sigma_{k-1}^{\epsilon}}(t)$ of $\tilde{F}$. It follows from the normalization of $\Phi_{1}^{-\epsilon}$ in Subsection 7.2.1 that $c_{\sigma_{k-1}^{\epsilon}(t)}$ is a boundary component of $\Phi_{1}^{(-1)^{k-1} \epsilon}$, and that $\dot{g}_{k-1}^{\epsilon}\left(c_{t}\right)$ is homotopic in $\Phi_{1}^{(-1)^{k-1} \epsilon}$ to $c_{\sigma_{k-1}^{\epsilon}}(t)$. Hence $\dot{h}_{k}^{\epsilon}\left(c_{t}\right)$ is homotopic in $\tilde{F}$ to $\dot{h}_{1}^{(-1)^{k-1} \epsilon}\left(c_{\sigma_{k-1}^{\epsilon}(t)}\right)$ which by the case $k=1$ of the lemma is equal to

$$
c_{\sigma_{1}^{(-1)^{k-1}} \epsilon_{\odot \sigma_{k-1}^{\epsilon}(t)}}=c_{\sigma_{k}^{\epsilon}(t)} .
$$

Since the boundary component $\dot{h}_{k}^{\epsilon}\left(c_{t}\right)$ of $\dot{h}_{k}^{\epsilon}\left(\dot{\Phi}_{k}^{\epsilon}\right)=\dot{\Phi}_{k}^{(-1)^{k+1} \epsilon}$ is homotopic to the component $c_{\sigma_{k}^{\epsilon}(t)}$ of $\partial \tilde{F}$, it follows from the normalization of $\dot{\Phi}_{k}^{(-1)^{k+1} \epsilon}$ that $\dot{h}_{k}^{\epsilon}\left(c_{t}\right)=c_{\sigma_{k}^{\epsilon}(t)}$.

Lemma 7.2.4. For each $\epsilon \in\{ \pm 1\}$ and each $k \geq 0$, there exists a homeomorphism $\breve{h}_{k}^{\epsilon}: \breve{\Phi}_{k}^{\epsilon} \rightarrow \breve{\Phi}_{k}^{(-1)^{k+1} \epsilon}$ such that

(1) the restriction of $\breve{h}_{k}^{\epsilon}$ to $\mathcal{L}\left(\breve{\Phi}_{k}^{\epsilon}\right)=\dot{\Phi}_{k}^{\epsilon}$ is $\dot{h}_{k}^{\epsilon}$;

(2) if $\tilde{F}$ is given a consistent orientation, $\breve{h}_{k}^{\epsilon}: \breve{\Phi}_{k}^{\epsilon} \rightarrow \tilde{F}$ reverses orientation if $k$ is odd and preserves orientation if $k$ is even; and 
(3) for each $t \in \mathbb{Z} / m \mathbb{Z}$ we have $\breve{h}_{k}^{\epsilon}\left(c_{t}\right)=c_{\sigma_{k}^{\epsilon}(t)}$.

The homeomorphism $\breve{h}_{k}^{\epsilon}$ is determined up to isotopy by the properties (1)-(3). Furthermore we may choose $\breve{h}_{1}^{\epsilon}$ within its isotopy class so that it is a free involution.

Proof. Fix an orientation of $\breve{\Phi}_{k}^{\epsilon}$ which is induced from a consistent orientation of $\tilde{F}$. By Lemma 7.2.3 we know that if $c_{t} \subset \dot{\Phi}_{k}^{\epsilon}$, then $\dot{h}_{k}^{\epsilon}\left(c_{t}\right)=c_{\sigma_{k}^{\epsilon}(t)}$. In particular, the correspondence $c_{t} \rightarrow c_{\sigma_{k}^{\epsilon}(t)}$ restricts to a bijection between the components of $\partial \tilde{F} \cap \dot{\Phi}_{k}^{\epsilon}$ and those of $\partial \tilde{F} \cap \dot{\Phi}_{k}^{(-1)^{k+1} \epsilon}$. It therefore also restricts to a bijection between the components of $\partial \tilde{F}-\partial \dot{\Phi}_{k}^{\epsilon}$ and those of $\partial \tilde{F}-\partial \dot{\Phi}_{k}(-1)^{k+1} \epsilon$. Now $\breve{\Phi}_{k}^{\epsilon}$ is the union of $\dot{\Phi}_{k}^{\epsilon}$ with the regular neighborhood $\mathcal{A}_{k}^{\epsilon}$ of $\partial \tilde{F}-\partial \dot{\Phi}_{k}^{\epsilon}$, and $\breve{\Phi}_{k}^{(-1)^{k+1} \epsilon}$ is the union of $\dot{\Phi}_{k}^{(-1)^{k+1} \epsilon}$ with the regular neighborhood $\mathcal{A}_{k+1}^{(-1)^{k+1} \epsilon}$ of $\partial \tilde{F}-\partial \dot{\Phi}_{k}^{(-1)^{k+1} \epsilon}$. Let $f_{k}^{\epsilon}$ be a homeomorphism from $\mathcal{A}_{k}^{\epsilon}$ to $\mathcal{A}_{k+1}^{(-1)^{k+1} \epsilon}$ which maps each component $c_{t}$ of $\partial \tilde{F} \cap \mathcal{A}_{k}^{\epsilon}$ to the component $c_{\sigma_{k}^{\epsilon}(t)}$ of $\partial \tilde{F} \cap \mathcal{A}_{k+1}^{(-1)^{k+1} \epsilon}$. Since each component of $\mathcal{A}_{k}^{\epsilon}$ is an annulus we may choose $f_{k}^{\epsilon}$ to be an orientation-reversing embedding of $\mathcal{A}_{k}^{\epsilon}$ into $\tilde{F}$ if $k$ is odd, and an orientation-preserving embedding if $k$ is even. These conditions determine $f_{k}$ up to isotopy. We define $\breve{h}_{k}^{\epsilon}$ to be the homeomorphism whose restriction to $\dot{\Phi}_{k}^{\epsilon}$ is $\dot{h}_{k}^{\epsilon}$ and whose restriction to $\mathcal{A}_{k}^{\epsilon}$ is $f_{k}$. Conditions (1) and (3) hold by construction. To see that condition (2) holds it suffices to observe that by Subsection 6.1 .5 the embedding $\dot{h}_{k}^{\epsilon}: \dot{\Phi}_{k}^{\epsilon} \rightarrow \tilde{F}$ reverses orientation if $k$ is odd and preserves orientation if $k$ is even, and that $f_{k}^{\epsilon}$ has the same property by construction.

Since we have observed that $f_{k}^{\epsilon}$ is determined up to isotopy by its stated properties, it follows that $\breve{h}_{k}^{\epsilon}$ is determined up to isotopy by conditions (1)-(3). Finally, since $\sigma_{1}^{\epsilon}$ is a free involution it is clear that we may choose $f_{1}^{\epsilon}$ within its isotopy class so that it is a free involution. Since $\dot{h}_{1}^{\epsilon}$ is a free involution by Subsection 6.1.5 it follows that $\breve{h}_{1}^{\epsilon}$ is a free involution.

7.2.5. For the rest of Subsection 7.2 we will fix homeomorphisms $\breve{h}_{k}^{\epsilon}$ satisfying the conclusions of Lemma 7.2.4. The free involution $\breve{h}_{1}^{\epsilon}$ will sometimes be denoted $\breve{\tau}_{\epsilon}$. Note that $\breve{\tau}_{\epsilon}$ is an extension of the free involution $\dot{\tau}_{\epsilon}$ defined in Subsection 6.1.5.

Lemma 7.2.6. Let $k$ and $i$ be integers with $k \geq i \geq 0$. Then $\breve{h}_{i}^{\epsilon}\left(\breve{\Phi}_{k}^{\epsilon}\right)$ is ambiently isotopic to $\mathcal{P}\left(\dot{h}_{i}^{\epsilon}\left(\dot{\Phi}_{k}^{\epsilon}\right)\right)$.

Proof. Since $\breve{\Phi}_{k}^{\epsilon}$ and $\breve{\Phi}_{i}^{\epsilon}$ are perfect surfaces, and since $\breve{h}_{i}^{\epsilon}: \breve{\Phi}_{i}^{\epsilon} \rightarrow \tilde{F}$ is an embedding which maps $\partial \tilde{F}$ onto $\partial \tilde{F}$ (see Lemma 7.2.3), it follows that $\breve{h}_{i}^{\epsilon}\left(\breve{\Phi}_{k}^{\epsilon}\right)$ is a perfect subsurface of $\tilde{F}$. On the other hand, by Subsections 7.1.2 and 7.2.1 we have $\dot{\Phi}_{k}^{\epsilon}=$ $\mathcal{L}\left(\breve{\Phi}_{k}^{\epsilon}\right)$. Since $\breve{h}_{i}^{\epsilon}$ is a homeomorphism, $\dot{h}_{i}^{\epsilon}\left(\dot{\Phi}_{k}^{\epsilon}\right)=\breve{h}_{i}^{\epsilon}\left(\dot{\Phi}_{k}^{\epsilon}\right)=\mathcal{L}\left(\breve{h}_{i}^{\epsilon}\left(\breve{\Phi}_{k}^{\epsilon}\right)\right)$. Since $\breve{h}_{i}^{\epsilon}\left(\breve{\Phi}_{k}^{\epsilon}\right)$ is perfect, it follows from Subsection 7.1.2 that $\breve{h}_{i}^{\epsilon}\left(\breve{\Phi}_{k}^{\epsilon}\right)$ is ambiently isotopic to $\mathcal{P}\left(\dot{h}_{i}^{\epsilon}\left(\dot{\Phi}_{k}^{\epsilon}\right)\right)$.

The next four results, Propositions 7.2.7 - 7.2.10, are analogues for the surfaces $\breve{\Phi}_{k}^{\epsilon}$ of Propositions 6.1.6, 6.1.7, 6.1.9 and 6.1.11.

Proposition 7.2.7. Let $i$ and $j$ be non-negative integers, and set $k=i+j$. Then for each $\epsilon \in\{ \pm 1\}$, the map $\breve{h}_{i}^{\epsilon} \mid \breve{\Phi}_{k}^{\epsilon}$ is homotopic in $\tilde{F}$, rel $\partial \tilde{F}$, to an embedding $\breve{g}_{i}^{\epsilon}: \breve{\Phi}_{k}^{\epsilon} \rightarrow \breve{\Phi}_{j}^{(-1)^{i} \epsilon}$ such that $\breve{h}_{j}^{(-1)^{i} \epsilon} \circ \breve{g}_{i}^{\epsilon}$ is homotopic in $\tilde{F}$ to $\breve{h}_{k}^{\epsilon}$. 
Proof. Let $\dot{g}_{i}^{\epsilon}: \dot{\Phi}_{k}^{\epsilon} \rightarrow \dot{\Phi}_{j}^{(-1)^{i} \epsilon}$ be given by Proposition 6.1.6. Let $c_{t}$ be a boundary component of $\tilde{F}$ that is contained in $\dot{\Phi}_{k}^{\epsilon}$. We have $\dot{h}_{i}^{\epsilon}\left(c_{t}\right)=c_{\sigma_{i}^{\epsilon}(t)}$. Since $\dot{g}_{i}^{\epsilon}$ is homotopic to $\dot{h}_{i}^{\epsilon}$, the map $\dot{g}_{i}^{\epsilon} \mid c_{t}: c_{t} \rightarrow \dot{\Phi}_{j}^{(-1)^{i} \epsilon}$ is homotopic in $\tilde{F}$ to $\dot{h}_{i}^{\epsilon} \mid c_{t}$. Because of the way that the surface $\dot{\Phi}_{j}^{(-1)^{i} \epsilon}$ has been normalized, this means that $c_{\sigma_{i}^{\epsilon}(t)}$ is a boundary curve of $\dot{\Phi}_{j}^{(-1)^{i} \epsilon}$ and that $\dot{g}_{i}^{\epsilon} \mid c_{t}$ is isotopic to $h_{i}^{\epsilon} \mid c_{t}$ in $\dot{\Phi}_{j}^{(-1)^{i} \epsilon}$. Therefore, after modifying $\dot{g}_{i}^{\epsilon}$ by a non-ambient isotopy, we may assume that, for each $c_{t}$ contained in $\partial \dot{\Phi}_{k}^{\epsilon}$, we have $\dot{g}_{i}^{\epsilon}\left(c_{t}\right)=c_{\sigma_{i}^{\epsilon}(t)}$ and $\dot{g}_{i}^{\epsilon}\left|c_{t}=\dot{h}_{i}^{\epsilon}\right| c_{t}$. Since the maps $\dot{g}_{i}^{\epsilon}$ and $\dot{h}_{i}^{\epsilon}$ are homotopic and agree on $\partial \tilde{F} \cap \dot{\Phi}_{k}^{\epsilon}$, they are homotopic rel $\partial \tilde{F}$.

The homeomorphism $\breve{h}_{i}^{\epsilon}$ maps $\mathcal{A}_{k}^{\epsilon}$ to a regular neighborhood of a collection of boundary curves of $\tilde{F}$. Since $\dot{g}_{i}^{\epsilon}$ agrees with $\breve{h}_{i}^{\epsilon}$ on $\dot{\Phi}_{k}^{\epsilon} \cap \partial \tilde{F}$, each boundary component of $\breve{h}_{i}^{\epsilon}\left(\mathcal{A}_{k}^{\epsilon}\right) \cap \partial \tilde{F}$ is disjoint from the image of $\dot{g}_{i}^{\epsilon}$. Thus $\breve{h}_{i}^{\epsilon} \mid \mathcal{A}_{k}^{\epsilon}$ is isotopic rel $\partial \tilde{F}$ to an embedding $f: \mathcal{A}_{k}^{\epsilon} \rightarrow \dot{\Phi}_{j}^{(-1)^{i} \epsilon}$ whose image is disjoint from the image of $\dot{g}_{i}^{\epsilon}$.

We define $\breve{g}_{i}^{\epsilon}$ so that $\breve{g}_{i}^{\epsilon} \mid \mathcal{A}_{k}^{\epsilon}=f$ and $\breve{g}_{i}^{\epsilon} \mid \dot{\Phi}_{k}^{\epsilon}=\dot{g}_{i}^{\epsilon}$. Then $\breve{g}_{i}^{\epsilon}$ is homotopic rel $\partial \tilde{F}$ to $\breve{h}_{i}^{\epsilon} \mid \breve{\Phi}_{k}^{\epsilon}$. Moreover we have $\breve{g}_{i}^{\epsilon}\left(c_{t}\right)=c_{\sigma_{i}^{\epsilon}(t)}$ for every $t \in \mathbb{Z} / m \mathbb{Z}$. Since $\sigma_{k}^{\epsilon}=\sigma_{j}^{(-1)^{i} \epsilon} \circ \sigma_{i}^{\epsilon}$ by (7.2.2.1), we have $\breve{h}_{j}^{(-1)^{i} \epsilon} \circ \breve{g}_{i}^{\epsilon}\left(c_{t}\right)=\breve{h}_{k}^{\epsilon}\left(c_{t}\right)$ for every $t \in \mathbb{Z} / m \mathbb{Z}$.

Let $\tilde{F}$ be given a consistent orientation. Since the embeddings $\breve{g}_{i}^{\epsilon}$ and $\breve{h}_{i}^{\epsilon} \mid \breve{\Phi}_{k}^{\epsilon}$ of $\breve{\Phi}_{k}^{\epsilon}$ into $\tilde{F}$ are homotopic rel $\partial \tilde{F}$, they both reverse orientation if $i$ is odd and preserve orientation if $i$ is even. In particular, since $i+j=k$, the embeddings $\breve{h}_{j}^{(-1)^{i} \epsilon} \circ \breve{g}_{i}^{\epsilon} \mid \mathcal{A}_{k}$ and $\breve{h}_{k}^{\epsilon} \mid \mathcal{A}_{k}$ are either both orientation preserving or both orientation reversing. Applying Lemma 7.1.10 to the restrictions of $\breve{h}_{j}^{(-1)^{i} \epsilon} \circ \breve{g}_{i}^{\epsilon}$ and $h_{k}^{\epsilon}$ to each component of $\mathcal{A}_{k}^{\epsilon}$ we conclude that $\breve{h}_{j}^{(-1)^{i} \epsilon} \circ \breve{g}_{i}^{\epsilon} \mid \mathcal{A}_{k}^{\epsilon}$ is homotopic to $\breve{h}_{k}^{\epsilon} \mid \mathcal{A}_{k}^{\epsilon}$. On the other hand, according to Proposition 6.1.6, $\dot{h}_{j}^{(-1)^{i} \epsilon} \circ \dot{g}_{i}^{\epsilon}$ is homotopic to $\dot{h}_{k}^{\epsilon}$. Hence $\breve{h}_{j}^{(-1)^{i} \epsilon} \circ \breve{g}_{i}^{\epsilon}$ is homotopic to $h_{k}^{\epsilon}$.

Proposition 7.2.8. Let $i$ and $j$ be non-negative integers, and set $k=i+j$. Then for each $\epsilon \in\{ \pm 1\}$ the subsurface $\breve{h}_{i}^{\epsilon}\left(\breve{\Phi}_{k}^{\epsilon}\right)$ is ambiently isotopic in $\tilde{F}$ to the perfect intersection $\breve{\Phi}_{i}^{(-1)^{i+1} \epsilon} \wedge_{\mathcal{P}} \breve{\Phi}_{j}^{(-1)^{i} \epsilon}$.

Proof. By definition we have

$$
\breve{\Phi}_{i}^{(-1)^{i+1} \epsilon} \wedge_{\mathcal{P}} \breve{\Phi}_{j}^{(-1)^{i} \epsilon}=\mathcal{P}\left(\mathcal{L}\left(\breve{\Phi}_{i}^{(-1)^{i+1} \epsilon}\right) \dot{\wedge}_{\mathcal{L}} \mathcal{L}\left(\breve{\Phi}_{j}^{(-1)^{i} \epsilon}\right)\right)=\mathcal{P}\left(\dot{\Phi}_{i}^{(-1)^{i+1} \epsilon} \dot{\wedge}_{\mathcal{L}} \dot{\Phi}_{j}^{(-1)^{i} \epsilon}\right) .
$$

Combining this with Proposition 6.1.7 we conclude that $\breve{\Phi}_{i}^{(-1)^{i+1} \epsilon} \wedge_{\mathcal{P}} \breve{\Phi}_{j}^{(-1)^{i} \epsilon}$ is equal to $\mathcal{P}\left(\dot{h}_{i}^{\epsilon}\left(\dot{\Phi}_{k}^{\epsilon}\right)\right)$ which, according to Lemma 7.2 .6 , is ambiently isotopic to $\breve{h}_{i}^{\epsilon}\left(\breve{\Phi}_{k}^{\epsilon}\right)$.

Proposition 7.2.9. For any non-negative integer $k$ and for each $\epsilon \in\{ \pm 1\}$ the surface $\breve{h}_{k}^{\epsilon}\left(\breve{\Phi}_{2 k+1}^{\epsilon}\right)$ is ambiently isotopic in $\tilde{F}$ to a subsurface of $\breve{\Phi}_{1}^{(-1)^{k} \epsilon}$ which is invariant under the free involution $\breve{\tau}_{(-1)^{k} \epsilon}$. In particular, $\breve{\Phi}_{2 k+1}^{\epsilon}$ admits a free involution which maps $c_{t}$ to $c_{\sigma_{2 k+1}^{\epsilon}(t)}$ for each $t \in \mathbb{Z} / m \mathbb{Z}$, and is orientation-reversing as an embedding of $\breve{\Phi}_{2 k+1}^{\epsilon}$ into $\tilde{F}$, if $\tilde{F}$ is given a consistent orientation.

Proof. According to Proposition 6.1.9 there is a subsurface $A$ of $\dot{\Phi}_{1}^{(-1)^{k} \epsilon} \subset \breve{\Phi}_{1}^{(-1)^{k} \epsilon}$ which is invariant under $\dot{\tau}_{(-1)^{k} \epsilon}=\breve{\tau}_{(-1)^{k} \epsilon} \mid \dot{\Phi}_{1}^{(-1)^{k} \epsilon}$ and isotopic to $\dot{h}_{k}^{\epsilon}\left(\dot{\Phi}_{2 k+1}^{\epsilon}\right)$. We 
apply Lemma 7.1 .3 , taking $S=\tilde{F}, P=\breve{\Phi}_{1}^{(-1)^{k} \epsilon}$, and $\tau=\breve{\tau}_{(-1)^{k} \epsilon}$. We conclude that $A$ has a perfection $Q \subset \breve{\Phi}_{1}^{(-1)^{k} \epsilon}$ which is invariant under $\breve{\tau}_{(-1)^{k} \epsilon}$. Since $Q$ is a perfection of $\dot{h}_{k}^{\epsilon}\left(\dot{\Phi}_{2 k+1}^{\epsilon}\right)$, it follows from Lemma 7.2.6 that $Q$ is ambiently isotopic to $\breve{h}_{k}^{\epsilon}\left(\breve{\Phi}_{2 k+1}^{\epsilon}\right)$. This completes the proof of the first assertion.

Let $h: \breve{\Phi}_{2 k+1}^{\epsilon} \rightarrow \tilde{F}$ be an embedding which is ambiently isotopic to $\breve{h}_{k}^{\epsilon} \mid \breve{\Phi}_{2 k+1}^{\epsilon}$ and maps $\breve{\Phi}_{2 k+1}^{\epsilon}$ onto $Q$. We define a free involution $\tau$ of $\breve{\Phi}_{2 k+1}^{\epsilon}$ by $\tau=h^{-1} \circ \breve{\tau}_{(-1)^{k} \epsilon} \circ h$. Thus $\tau\left(c_{t}\right)=c_{s}$ where $s=\left(\sigma_{k}^{\epsilon}\right)^{-1} \circ \sigma_{1}^{(-1)^{k} \epsilon} \circ \sigma_{k}^{\epsilon}(t)$. It follows from (7.2.2.1) that $s=\sigma_{2 k+1}^{\epsilon}(t)$.

Let $\tilde{F}$ be given a consistent orientation. Lemma 7.2.4 implies that $h$ preserves orientation if $k$ is odd, that $h$ reverses orientation if $k$ is even, and that $\breve{\tau}_{(-1)^{k} \epsilon}$ reverses orientation. It follows that $\tau$ is orientation-reversing as an embedding of $\breve{\Phi}_{2 k+1}^{\epsilon}$ into $\tilde{F}$.

Proposition 7.2.10. Let $k$ be a non-negative integer and let $\epsilon \in\{ \pm 1\}$ be given. If $\breve{\Phi}_{k}^{\epsilon}$ and $\breve{\Phi}_{k+2}^{\epsilon}$ are isotopic in $\tilde{F}$, then either $\breve{\Phi}_{k}^{\epsilon}$ is a regular neighborhood of $\partial \tilde{F}$ or $\tilde{F}$ is a semi-fiber.

Proof. By definition we have that $\breve{\Phi}_{k}^{\epsilon}=\mathcal{P}\left(\dot{\Phi}_{k}^{\epsilon}\right)$ and $\breve{\Phi}_{k+2}^{\epsilon}=\mathcal{P}\left(\dot{\Phi}_{k+2}^{\epsilon}\right)$. Thus it follows from Subsection 7.1.2 that $\breve{\Phi}_{k}^{\epsilon}$ is isotopic to $\breve{\Phi}_{k+2}^{\epsilon}$ if and only if $\dot{\Phi}_{k}^{\epsilon}$ is isotopic to $\dot{\Phi}_{k+2}^{\epsilon}$, and that $\breve{\Phi}_{k}^{\epsilon}$ is a regular neighborhood of $\partial \tilde{F}$ if and only if $\dot{\Phi}_{k}^{\epsilon}$ is empty. The result therefore follows from Proposition 6.1.11.

7.3. Very tight surfaces. In this subsection we assume that $M$ is a simple knot manifold, and that $\tilde{F}$ is a splitting surface for $M$ which admits a long rectangle.

For each integer $k \geq 0$ and each $\epsilon \in\{ \pm 1\}$ we will define the perfect subsurfaces $\breve{\Phi}_{k}^{\epsilon}=\mathcal{A}_{k}^{\epsilon} \cup \dot{\Phi}_{k}^{\epsilon}$, with $\tilde{F}=\breve{\Phi}_{0}^{\epsilon} \supset \breve{\Phi}_{1}^{\epsilon} \supset \breve{\Phi}_{2}^{\epsilon} \supset \cdots$, as in Subsection 7.2.

We set $m=|\partial \tilde{F}|$ and we assume that the components of $\partial \tilde{F}$ have been indexed by elements of $\mathbb{Z} / m \mathbb{Z}$ as described in Subsection 7.2. We also define the permutations $\sigma_{k}^{\epsilon}$ as in Subsection 7.2, and for each integer $k \geq 0$ and $\epsilon \in\{ \pm 1\}$ we fix a homeomorphism $\breve{h}_{k}^{\epsilon}$ satisfying the conclusions of Lemma 7.2.4.

For $\epsilon \in\{ \pm 1\}$ we denote by $\mathcal{T}^{\epsilon}$ the set of tight components of $\breve{\Phi}_{1}^{\epsilon}$. We define $s_{0}$ to be the infimum of $s(C)$ as $C$ ranges over $\mathcal{T}^{+} \cup \mathcal{T}^{-}$. Thus, if $\mathcal{T}^{+}=\mathcal{T}^{-}=\emptyset$, then $s_{0}=+\infty$ and otherwise $s_{0}$ is a strictly positive integer. We will say that a component of a perfect subsurface of $\tilde{F}$ is very tight if it is tight and has size at most $s_{0}$. (In particular, if $\mathcal{T}^{+}=\mathcal{T}^{-}=\emptyset$, then any tight component of a perfect subsurface is very tight.)

If $A$ is a perfect subsurface of $\tilde{F}$, then we define $V T(A)$ to be the union of the very tight components of $A$.

Lemma 7.3.1. If $\epsilon \in\{ \pm 1\}$ and $k>0$ is odd, then $\left|V T\left(\breve{\Phi}_{k}^{\epsilon}\right)\right|$ is even.

Proof. We give $\breve{\Phi}_{k}^{\epsilon}$ the orientation inherited from a consistent orientation of $\tilde{F}$. Since $k$ is odd, Proposition 7.2.9 implies that the surface $\breve{\Phi}_{k}^{\epsilon}$ admits an orientationreversing free involution $\tau$ which permutes the components of $\partial \tilde{F}$. It follows that if $T$ is a very tight component of $\breve{\Phi}_{k}^{\epsilon}$, then $\tau(T)$ is also a very tight component of $\breve{\Phi}_{k}^{\epsilon}$, and that $\tau$ maps the (connected) frontier of $T$ to the frontier of $\tau(T)$. Since a free orientation-reversing involution of an oriented surface cannot leave any boundary 
component invariant, we conclude that no tight component of $\breve{\Phi}_{k}^{\epsilon}$ can be invariant under $\tau$. Thus the number of tight components must be even.

Lemma 7.3.2. Let $\epsilon \in\{ \pm 1\}$ and let $k$ be a non-negative integer. Let $T$ be a very tight component of $\breve{\Phi}_{k}^{\epsilon}$. Then every component of $\breve{\Phi}_{k+1}^{\epsilon}$ which is contained in $T$ is very tight. Moreover if $T$ contains exactly one component $X$ of $\breve{\Phi}_{k+1}^{\epsilon}$, then $T^{\prime}$ is ambiently isotopic to $T$.

Proof. Since $\partial \tilde{F} \subset \breve{\Phi}_{k}^{\epsilon}$ is invariant under the homeomorphism $\breve{h}_{k}^{\epsilon}$, the component $\breve{h}_{k}^{\epsilon}(T)$ of $\breve{\Phi}_{k}^{(-1)^{k+1} \epsilon}$ is very tight. According to Proposition $7.2 .8, \breve{h}_{k}^{\epsilon}\left(\breve{\Phi}_{k+1}^{\epsilon}\right)$ is ambiently isotopic to the perfect intersection $\breve{\Phi}_{k}^{(-1)^{k+1} \epsilon} \wedge_{\mathcal{P}} \breve{\Phi}_{1}^{-\epsilon}$.

Thus if $X$ is a component of $\breve{\Phi}_{k+1}^{\epsilon}$ which is contained in $T$, then $\breve{h}_{k}^{\epsilon}(X)$ is

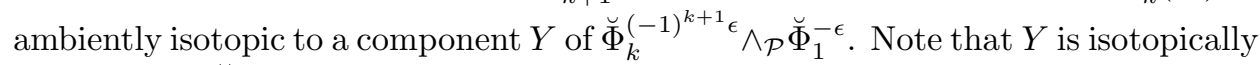
contained in $\breve{h}_{k}^{\epsilon}(T)$. According to the definition of $s_{0}$, every tight component of $\breve{\Phi}_{1}^{-\epsilon}$ has size at least $s_{0}$, and $s(T) \leq s_{0}$ by the definition of a very tight subsurface. Applying Proposition 7.1.8 with $P=\breve{\Phi}_{k}^{(-1)^{k+1} \epsilon}, P_{0}=\breve{h}_{k}^{\epsilon}(T)$, and $Q=\breve{\Phi}_{1}^{-\epsilon}$, we conclude that if a component of $\breve{\Phi}_{k}^{(-1)^{k+1} \epsilon} \wedge_{\mathcal{P}} \breve{\Phi}_{1}^{-\epsilon}$ is isotopically contained in $\breve{h}_{k}^{\epsilon}(T)$, then it is tight of size at most $s(T)$, and therefore is very tight. This shows that $Y$ is very tight.

Now suppose that $T$ contains exactly one component $X$ of $\breve{\Phi}_{k+1}^{\epsilon}$. Again $\breve{h}_{k}^{\epsilon}(X)$ is ambiently isotopic to a component $Y$ of $\breve{\Phi}_{k}^{(-1)^{k+1} \epsilon} \wedge_{\mathcal{P}} \breve{\Phi}_{1}^{-\epsilon}$. Since $X=\breve{\Phi}_{k+1}^{\epsilon} \cap T$, and since $\partial \tilde{F} \subset \breve{\Phi}_{k+1}^{\epsilon}$, we have $T \cap \partial \tilde{F}=X \cap \partial \tilde{F}$. Since $\partial \tilde{F}$ is invariant under the homeomorphism $\breve{h}_{k}^{\epsilon}$, we have $\breve{h}_{k}^{\epsilon}(T) \cap \partial \tilde{F}=h_{k}^{\epsilon}(X) \cap \partial \tilde{F}=Y \cap \partial \tilde{F}$. But any component of $\breve{\Phi}_{k}^{(-1)^{k+1} \epsilon} \wedge_{\mathcal{P}} \breve{\Phi}_{1}^{-\epsilon}$ which is isotopically contained in $\breve{h}_{k}^{\epsilon}(T)$ must contain some component of $\breve{h}_{k}^{\epsilon}(T) \cap \partial \tilde{F} \subset Y$. Thus $Y$ is the only component of $\breve{\Phi}_{k}^{(-1)^{k+1} \epsilon} \wedge_{\mathcal{P}} \breve{\Phi}_{1}^{-\epsilon}$ which is isotopically contained in $\breve{h}_{k}^{\epsilon}(T)$. Proposition 7.1.8 now implies that $\breve{h}_{k}^{\epsilon}(T)$ is ambiently isotopic to $Y$, and hence to $\breve{h}_{k}^{\epsilon}(X)$. Applying the inverse of the homeomorphism $\breve{h}_{k}^{\epsilon}$, we conclude that $X$ is ambiently isotopic to $T$.

Lemma 7.3.3. Let $\epsilon \in\{ \pm 1\}$ be given and let $l \geq k \geq 0$ be integers. Then each very tight component of $\breve{\Phi}_{k}^{\epsilon}$ contains at least one very tight component of $\breve{\Phi}_{l}^{\epsilon}$. In particular $\left|V T\left(\breve{\Phi}_{l}^{\epsilon}\right)\right| \geq\left|V T\left(\breve{\Phi}_{k}^{\epsilon}\right)\right|$.

Proof. It suffices to consider the case $l=k+1$. Since $\breve{\Phi}_{k}^{\epsilon} \supset \breve{\Phi}_{k+1}^{\epsilon} \supset \partial \tilde{F}$, and since each component of $\breve{\Phi}_{k}^{\epsilon}$ meets $\partial \tilde{F}$, each component of $\breve{\Phi}_{k}^{\epsilon}$ must contain at least one component of $\breve{\Phi}_{k+1}^{\epsilon}$. The assertion therefore follows from Lemma 7.3.2.

Lemma 7.3.4. Let $\epsilon \in\{ \pm 1\}$ be given and let $l \geq k$ be non-negative integers. If $\left|V T\left(\breve{\Phi}_{l}^{\epsilon}\right)\right|=\left|V T\left(\breve{\Phi}_{k}^{\epsilon}\right)\right|$, then $\operatorname{VT}\left(\breve{\Phi}_{l}^{\epsilon}\right)$ is isotopic to $\operatorname{VT}\left(\breve{\Phi}_{k}^{\epsilon}\right)$.

Proof. If $l>k$ and $\left|V T\left(\breve{\Phi}_{l}^{\epsilon}\right)\right|=\left|V T\left(\breve{\Phi}_{k}^{\epsilon}\right)\right|$, then by Lemma 7.3 .3 we have

$$
\left|V T\left(\breve{\Phi}_{k}^{\epsilon}\right)\right|=\left|V T\left(\breve{\Phi}_{k+1}^{\epsilon}\right)\right|=\cdots=\left|V T\left(\breve{\Phi}_{l}^{\epsilon}\right)\right| .
$$

It thus suffices to show that if $\left|V T\left(\breve{\Phi}_{k+1}^{\epsilon}\right)\right|=\left|V T\left(\breve{\Phi}_{k}^{\epsilon}\right)\right|$, then $V T\left(\breve{\Phi}_{k+1}^{\epsilon}\right)$ is isotopic to $V T\left(\breve{\Phi}_{k}^{\epsilon}\right)$. 
By Lemma 7.3.3, if $\left|V T\left(\breve{\Phi}_{k+1}^{\epsilon}\right)\right|=\left|V T\left(\breve{\Phi}_{k}^{\epsilon}\right)\right|$, then each very tight component of $\breve{\Phi}_{k}^{\epsilon}$ contains exactly one very tight component of $\breve{\Phi}_{k+1}^{\epsilon}$, and each very tight component of $\breve{\Phi}_{k+1}^{\epsilon}$ is contained in a very tight component of $\breve{\Phi}_{k}^{\epsilon}$. Thus Lemma 7.3.2 implies that $\operatorname{VT}\left(\breve{\Phi}_{k+1}^{\epsilon}\right)$ is isotopic to $\operatorname{VT}\left(\breve{\Phi}_{k}^{\epsilon}\right)$.

Lemma 7.3.5. Let $\epsilon \in\{ \pm 1\}$ and $t \in \mathbb{Z} / m \mathbb{Z}$ be given, and let $k \geq 0$ be an integer. If $c_{\sigma_{2}^{\epsilon}(t)}$ is contained in $\operatorname{VT}\left(\breve{\Phi}_{k}^{\epsilon}\right)$, then $c_{t}$ is contained in $V T\left(\breve{\Phi}_{k+2}^{\epsilon}\right)$.

Proof. Let $C$ be the component of $\breve{\Phi}_{k+2}^{\epsilon}$ which contains $c_{t}$. Then $\breve{h}_{2}^{\epsilon}(C)$ is the component of $\breve{h}_{2}^{\epsilon}\left(\Phi_{k+2}^{\epsilon}\right)$ which contains $c_{\sigma_{2}^{\epsilon}(t)}$. Since the embedding $\breve{h}_{2}^{\epsilon}$ maps $\partial \tilde{F}$ to $\partial \tilde{F}$, the subsurface $C$ is very tight if and only if $\breve{h}_{2}^{\epsilon}(C)$ is very tight. According to Proposition 7.2.8, $\breve{h}_{2}^{\epsilon}\left(\Phi_{k+2}^{\epsilon}\right)$ is isotopic to the perfect intersection $\breve{\Phi}_{2}^{-\epsilon} \wedge_{\mathcal{P}} \breve{\Phi}_{k}^{\epsilon}$. By Subsection 7.1.6 $\breve{h}_{2}^{\epsilon}(C)$ is isotopically contained in a unique component $C^{\prime}$ of $\breve{\Phi}_{k}^{\epsilon}$, which must be the component of $\breve{\Phi}_{k}^{\epsilon}$ that contains $c_{\sigma_{2}^{\epsilon}(t)}$. Thus $C^{\prime}$ is very tight. By Proposition 7.1.8, every component of $\breve{\Phi}_{2}^{-\epsilon} \wedge_{\mathcal{P}} \breve{\Phi}_{k}^{\epsilon}$ which is contained in $C^{\prime}$ is very tight. This shows that $\breve{h}_{2}^{\epsilon}(C)$ is very tight, as required.

Lemma 7.3.6. Let $\epsilon \in\{ \pm 1\}$ be given and let $k>0$ be an odd integer. If $\left|V T\left(\breve{\Phi}_{k+2}^{\epsilon}\right)\right|=\left|V T\left(\breve{\Phi}_{k}^{\epsilon}\right)\right|>0$, then $\breve{\Phi}_{k+2}^{\epsilon}$ is ambiently isotopic to $\breve{\Phi}_{k}^{\epsilon}$.

Proof. Assume that $\left|V T\left(\breve{\Phi}_{k+2}^{\epsilon}\right)\right|=\left|V T\left(\breve{\Phi}_{k}^{\epsilon}\right)\right|>0$.

By Lemma 7.3.4 we have that $V T\left(\breve{\Phi}_{k+2}^{\epsilon}\right)$ is isotopic to $V T\left(\breve{\Phi}_{k}^{\epsilon}\right)$. By Lemma 7.3.5 this implies that if $c_{\sigma_{2}^{\epsilon}(t)}$ is contained in $V T\left(\breve{\Phi}_{k}^{\epsilon}\right)$, then so is $c_{t}$. Since $V T\left(\breve{\Phi}_{k}^{\epsilon}\right) \neq \emptyset$ it follows that either $c_{\bar{q}} \subset V T\left(\breve{\Phi}_{k}^{\epsilon}\right)$ for every even integer $q$, or else $c_{\bar{q}} \subset V T\left(\breve{\Phi}_{k}^{\epsilon}\right)$ for every odd integer $q$. But $V T\left(\breve{\Phi}_{k}^{\epsilon}\right)$ is invariant under the free involution $\tau_{k}^{\epsilon}$, which maps each boundary curve $c_{\bar{q}}$ to a boundary curve $c_{\bar{r}}$, where $q$ and $r$ have opposite parity. Thus every boundary component of $\tilde{F}$ is contained in $V T\left(\breve{\Phi}_{k}^{\epsilon}\right)$. Since every component of $\breve{\Phi}_{k}^{\epsilon}$ contains a component of $\partial \tilde{F}$, it follows that $V T\left(\breve{\Phi}_{k}^{\epsilon}\right)=\breve{\Phi}_{k}^{\epsilon}$. Since $\breve{\Phi}_{k+2}^{\epsilon} \subset \breve{\Phi}_{k}^{\epsilon}$, and since Lemma 7.3.2 implies that every component of $\breve{\Phi}_{k+2}^{\epsilon}$ which is contained in a tight component of $\breve{\Phi}_{k}^{\epsilon}$ is tight, we conclude that $V T\left(\breve{\Phi}_{k+2}^{\epsilon}\right)=\breve{\Phi}_{k+2}^{\epsilon}$. Hence $\breve{\Phi}_{k}^{\epsilon}$ is ambiently isotopic to $\breve{\Phi}_{k+2}^{\epsilon}$.

Lemma 7.3.7. Suppose that $\tilde{F}$ is not a semi-fiber. Let $\epsilon \in\{ \pm 1\}$ be given and let $k>0$ be an odd integer. If $\left|V T\left(\breve{\Phi}_{k+2}^{\epsilon}\right)\right|=\left|V T\left(\breve{\Phi}_{k}^{\epsilon}\right)\right|>0$, then $\breve{\Phi}_{k}^{\epsilon}$ is a regular neighborhood of $\partial \tilde{F}$.

Proof. This is an immediate consequence of Proposition 7.2.10 and Lemma 7.3.6.

Proposition 7.3.8. Suppose that $\tilde{F}$ is not a semi-fiber. Let $p>0$ be an odd integer and suppose that either $\breve{\Phi}_{p}^{+}$or $\breve{\Phi}_{p}^{-}$has a tight component. Then either $\breve{\Phi}_{p+m-2}^{+}$or $\breve{\Phi}_{p+m-2}^{-}$is a regular neighborhood of $\partial \tilde{F}$.

Proof. We can assume without loss of generality that $p$ is the smallest odd integer such that either $\breve{\Phi}_{p}^{+}$or $\breve{\Phi}_{p}^{-}$has a tight component. We claim that either $\breve{\Phi}_{p}^{+}$or $\breve{\Phi}_{p}^{-}$ has a very tight component. If $p=1$, then, by the definition of $s_{0}$, either $\breve{\Phi}_{1}^{+}$or $\breve{\Phi}_{1}^{-}$has a component of size $s_{0}$, which is very tight by the definition of a very tight 
component. If $p>1$, then $s_{0}=\infty$ and any tight component is very tight, so the claim is true in this case as well.

Now fix $\epsilon \in\{ \pm 1\}$ such that $\breve{\Phi}_{p}^{\epsilon}$ has a very tight component. It follows from Lemma 7.3.1 that $\left|V T\left(\breve{\Phi}_{p}^{\epsilon}\right)\right| \geq 2$. We will show that $\breve{\Phi}_{p+m-2}^{\epsilon}$ is a regular neighborhood of $\partial \tilde{F}$. If there is an even integer $k$ with $0<k<m-2$ such that $\breve{\Phi}_{p+k}^{\epsilon}$ is a regular neighborhood of $\partial \tilde{F}$, then the conclusion holds because $\partial \tilde{F} \subset \breve{\Phi}_{p+m-2}^{\epsilon} \subset \breve{\Phi}_{p+k}^{\epsilon}$.

Now suppose that there is no even integer $k$ with $0<k<m-2$ such that $\breve{\Phi}_{p+k}^{\epsilon}$ is a regular neighborhood of $\partial \tilde{F}$. Since $\tilde{F}$ is not a semi-fiber, for all even $k$ with $0<k<m-2$ we have that $\left|V T\left(\breve{\Phi}_{p+k+2}^{\epsilon}\right)\right|>\left|V T\left(\breve{\Phi}_{p+k}^{\epsilon}\right)\right|$ by Lemma 7.3 .3 and Lemma 7.3.7, and hence, by Lemma 7.3.1, that $\left|V T\left(\breve{\Phi}_{p+k+2}^{\epsilon}\right)\right| \geq\left|V T\left(\breve{\Phi}_{p+k}^{\epsilon}\right)\right|+2$. Since $\left|V T\left(\breve{\Phi}_{p}^{\epsilon}\right)\right| \geq 2$ it follows that $\left|V T\left(\breve{\Phi}_{p+m-2}^{\epsilon}\right)\right| \geq m$.

In particular $\breve{\Phi}_{p+m-2}$ has at least $m$ tight components. Each tight component has size at least 1 , and the sum of the sizes is at most $m=|\partial \tilde{F}|$. Thus $\breve{\Phi}_{p+m-2}$ has $m$ tight components of size exactly 1 , which are therefore regular neighborhoods of components of $\partial \tilde{F}$. Since $\breve{\Phi}_{p+m-2}^{\epsilon}$ is perfect it must be a regular neighborhood of $\partial \tilde{F}$.

Corollary 7.3.9. Suppose that $\tilde{F}$ is not a semi-fiber. Let $p>0$ be an odd integer and suppose that either $\breve{\Phi}_{p}^{+}$or $\breve{\Phi}_{p}^{-}$has a tight component. Then both $\breve{\Phi}_{p+m-1}^{+}$and $\breve{\Phi}_{p+m-1}^{-}$are regular neighborhoods of $\partial \tilde{F}$.

Proof. By Proposition 7.3.8 we know that, for some $\epsilon \in\{ \pm 1\}, \breve{\Phi}_{p+m-2}^{\epsilon}$ is a regular neighborhood of $\partial \tilde{F}$. Since the $\breve{\Phi}_{k}^{\epsilon}$ are nested perfect surfaces, $\breve{\Phi}_{p+m-1}^{\epsilon}$ is also a regular neighborhood of $\partial \tilde{F}$. By Proposition 7.2 .8 the subsurface $\breve{\Phi}_{p+m-1}^{-\epsilon}$ is mapped homeomorphically by $\breve{h}_{1}^{-\epsilon}$ to $\breve{\Phi}_{1}^{-\epsilon} \wedge_{\mathcal{P}} \breve{\Phi}_{p+m-2}^{\epsilon}$, which is isotopically contained in $\breve{\Phi}_{p+m-2}^{\epsilon}$. Since $\partial \tilde{F}$ is invariant under $\breve{h}_{1}^{-\epsilon}$ it follows that $\breve{\Phi}_{p+m-1}^{-\epsilon}$ is a regular neighborhood of $\partial \tilde{F}$.

\subsection{Planar essential surfaces and their boundary slopes.}

Theorem 7.4.1. Let $F$ be an essential planar surface in a simple knot manifold $M$. Suppose that $F$ is not a semi-fiber. Set $m=|\partial F|$ and let $H$ be any reduced homotopy in the pair $(M, F)$ such that $H_{0}$ is an essential path in $F$ and $H_{t}(\partial I) \subset \partial M$ for each $t \in I$. Then the length of $H$ is at most $m-1$.

Proof. We shall assume that the length of $H$ is at least $m$ and derive a contradiction. Let $\tilde{F}$ be the splitting surface associated to $F$ (see Subsection 5.4.2). Set $\tilde{m}=$ $|\partial \tilde{F}|$, so $\tilde{m}=m$ if $F$ is separating and $\tilde{m}=2 m$ if $F$ is non-separating. The homotopy $H$ determines a reduced homotopy $\tilde{H}$ in the pair $(M, \tilde{F})$ of length at least $\tilde{m}$ such that $\tilde{H}_{0}$ is an essential path in $\tilde{F}$ and $\tilde{H}_{t}(\partial I) \subset \partial M$ for each $t \in I$. In particular, $\tilde{F}$ admits a long rectangle. Thus the assumptions of Subsections 6.1 and 7.2 hold in our situation and we may use the notation and apply the results from those subsections.

Since the surface $F$ is not a semi-fiber, it follows that $\tilde{F}$ is also not a semi-fiber and hence, by Proposition 5.3.9, that there exists $\epsilon \in\{ \pm 1\}$ such that $\tilde{F}$ is not a regular neighborhood of $\Phi_{1}^{\epsilon}$. This implies that $\breve{\Phi}_{1}^{\epsilon}$ is a proper subsurface of $\tilde{F}$. Thus Proposition 7.1.9 implies that $\breve{\Phi}_{1}^{\epsilon}$ has a tight component. We conclude from 
Proposition 7.3.8 that $\breve{\Phi}_{\tilde{m}}^{+}$and $\breve{\Phi}_{\tilde{m}}^{-}$are regular neighborhoods of $\partial \tilde{F}$. Hence $\dot{\Phi}_{\tilde{m}}^{+}$ and $\dot{\Phi}_{\tilde{m}}^{-}$are empty.

On the other hand, since the reduced homotopy $\tilde{H}$ has length at least $\tilde{m}$, it follows from Subsection 3.4 and Proposition 5.2.8 that there is an admissible pair of glasses $\gamma: \Gamma \rightarrow \tilde{F}$ which is homotopic in $\tilde{F}$ to a map from $\Gamma$ to $\Phi_{\tilde{m}}^{\epsilon}$. In particular there is a map $\alpha: S^{1} \rightarrow \partial \tilde{F}$ which is homotopic in $\tilde{F}$ to a map from $S^{1}$ to a component $A$ of $\Phi_{\tilde{m}}^{\epsilon}$. It follows that $A$ must be an outer component of $\Phi_{\tilde{m}}^{\epsilon}$, and hence that $\dot{\Phi}_{\tilde{m}}^{\epsilon} \neq \emptyset$. This contradiction completes the proof.

Theorem 7.4.2. Let $M$ be a simple knot manifold and let $F \subset M$ be an essential planar surface with boundary slope $\beta$ which is not a semi-fiber. Let $(S, X, h)$ be a singular surface which is well positioned with respect to $F$ and has boundary slope $\alpha$. Set $s=$ genus $S, n=|\partial S-X|, v=|X|$. Then

$$
\Delta(\alpha, \beta) \leq N(s, n, v) .
$$

Proof. Set $m=|\partial F|$. According to Proposition 3.9, there exists an essential homotopy $H: I \times I \rightarrow M$ having length

$$
l \geq \frac{m \Delta(\alpha, \beta)}{N(s, n, v)}-1,
$$

such that $H_{0}$ is an essential path in $F$ and $H_{t}(\partial I) \subset \partial M$ for all $t \in I$. By Theorem 7.4.1 we have that

$$
l \leq m-1 .
$$

The conclusion follows from inequalities (1) and (2).

Corollary 7.4.3. Let $M$ be a simple knot manifold, and let $F \subset M$ be an essential planar surface with boundary slope $\beta$ which is not a semi-fiber. Let $\alpha$ be a slope in $\partial M$. If $M(\alpha)$ is very small, or more generally if $F \subset M \subset M(\alpha)$ is not $\pi_{1}$-injective in $M(\alpha)$, then

$$
\Delta(\alpha, \beta) \leq 5 .
$$

Proof. We invoke Corollary 2.3 to obtain a singular surface $(S, X, h)$, well positioned with respect to $F$, such that genus $S=0$ and $|X|=1$. The conclusion now follows from Theorem 7.4.2 because for any $v \geq 1$ we have $N(0,1, v) \leq 5$.

Corollary 7.4.4. Let $M$ be a simple knot manifold. Suppose that $M(\beta)$ is a reducible manifold which is not homeomorphic to $S^{1} \times S^{2}$ or $P^{3} \# P^{3}$ and that $M(\alpha)$ is very small. Then

$$
\Delta(\alpha, \beta) \leq 5 .
$$

Proof. Since $M(\beta)$ is reducible, $M$ contains an essential planar surface $F$ with boundary slope $\beta$. If $F$ is a semi-fiber, then $M(\beta)$ is homeomorphic to either $S^{1} \times S^{2}$ or $P^{3} \# P^{3}$. Thus the corollary follows from Corollary 7.4.3.

Corollary 7.4.5. Let $M$ be a simple knot manifold, and let $F \subset M$ be an essential planar surface with boundary slope $\beta$ which is not a semi-fiber. Let $\alpha$ be a slope in $\partial M$. If $M(\alpha)$ is a Seifert fibered space or if there exists a $\pi_{1}$-injective map from $S^{1} \times S^{1}$ to $M(\alpha)$, then

$$
\Delta(\alpha, \beta) \leq 6 .
$$


Proof. We invoke Corollary 2.6 to obtain a singular surface $(S, X, h)$, well positioned with respect to $F$, such that either genus $S=0$ and $|X|=1$, or genus $S=1$ and $|X|=0$. The conclusion now follows from Theorem 7.4.2 because for any $v \geq 1$ we have $N(0,1, v) \leq 5$ and $N(1,0, v)=6$.

Corollary 7.4.6. Let $M$ be a simple knot manifold. Suppose that $M(\beta)$ is a reducible manifold which is not homeomorphic to $S^{1} \times S^{2}$ or $P^{3} \# P^{3}$ and that $M(\alpha)$ is Seifert fibered. Then

$$
\Delta(\alpha, \beta) \leq 6 .
$$

Proof. As in the proof of Corollary 7.4.4 we apply Corollary 7.4.5 to the planar surface obtained by intersecting a reducing sphere for $M(\beta)$ with $M$.

The following corollary to Theorem 7.4.2 is a special case of a result of Gordon and Litherland [GLi Proposition 6.1], which has the same upper bound, but with a strict inequality and without the assumption that the essential planar surface is not a semi-fiber.

Corollary 7.4.7. Let $M$ be a simple knot manifold, and let $F \subset M$ be an essential planar surface with boundary slope $\beta$ which is not a semi-fiber. Suppose that $S \subset M$ is an essential bounded surface of genus $g$ with boundary slope $\alpha$. Set $m=|\partial S|$. Then we have

$$
\Delta(\alpha, \beta) \leq\left[\frac{12 g-12}{m}\right]+6 .
$$

Proof. We apply Proposition 2.1 to obtain a singular surface $\left(S^{\prime}, \partial S^{\prime}, h\right)$ which is well positioned with respect to $F$. Theorem 7.4.2 then implies that

$$
\Delta(\alpha, \beta) \leq N(g, 0, m)
$$

Note that since $M$ is a simple knot manifold, the surface $S^{\prime}$ cannot be a disk or an annulus. It then follows from Subsection 3.7 that

$$
N(s, n, v)=\left[\frac{12 g-12}{m}\right]+6 .
$$

\section{SEIFERT FIBERED SURGERIES}

According to Corollary 7.4.6, if $M$ is a simple knot manifold and if $\alpha$ and $\beta$ are slopes such that $M(\alpha)$ is Seifert fibered while $M(\beta)$ is reducible but is not $S^{1} \times S^{2}$ or $P^{3} \# P^{3}$, then $\Delta(\alpha, \beta) \leq 6$. In fact we know of no examples where $\Delta(\alpha, \beta)>3$. In this section, building on Corollary 7.4.6, we prove a result, Proposition 8.4, which gives restrictions on the possible Seifert fibrations of $M(\alpha)$ in the cases where $\Delta(\alpha, \beta)>3$, which shows that this situation is not generic. The proof uses the character variety of $M$ and some observations from algebraic number theory. We will see that in the generic situation, 3 is an upper bound for the distance between $\alpha$ and $\beta$. A similar result, Proposition 8.7 applies to the case where $M(\alpha)$ is a Seifert fibered space that contains an incompressible torus, $M(\alpha)$ is Seifert fibered and $\Delta(\alpha, \beta)>5$. Here, in place of Corollary 7.4.6 we use a theorem of Agol [A] and Lackenby [La] which implies that $\Delta(\alpha, \beta) \leq 10$ in this situation. 
For any integer $n \geq 1$, we set $\zeta_{n}=e^{\frac{2 \pi i}{n}}$.

Lemma 8.1. Let $m, n \geq 1$ be integers. If $\zeta_{n}+\bar{\zeta}_{n} \in \mathbb{Q}\left(\zeta_{m}\right)$, then one of the following three conditions holds:

(i) $n \in\{1,2,3,4,6\}$.

(ii) $n$ divides $m$.

(iii) $\frac{n}{2}$ is an odd integer dividing $m$.

Proof. Without loss of generality we take $n \notin\{1,2,3,4,6\}$, so $\zeta_{n}+\bar{\zeta}_{n} \notin \mathbb{Q}$. Let $d=\operatorname{gcd}(n, m)$. By hypothesis

$$
\zeta_{n}+\bar{\zeta}_{n} \in \mathbb{Q}\left(\zeta_{m}\right) \cap \mathbb{Q}\left(\zeta_{n}\right)=\mathbb{Q}\left(\zeta_{d}\right)
$$

$([\mathrm{FT}, \mathrm{VI} .2 .8])$ so that $\zeta_{n}+\bar{\zeta}_{n} \in \mathbb{Q}\left(\zeta_{d}\right)_{\mathbb{R}}$. Thus

$$
\mathbb{Q}\left(\zeta_{n}\right)_{\mathbb{R}}=\mathbb{Q}\left(\zeta_{n}+\bar{\zeta}_{n}\right) \subset \mathbb{Q}\left(\zeta_{d}\right)_{\mathbb{R}}
$$

But clearly $\mathbb{Q}\left(\zeta_{d}\right)_{\mathbb{R}} \subset \mathbb{Q}\left(\zeta_{n}\right)_{\mathbb{R}}$ and therefore $\mathbb{Q}\left(\zeta_{d}\right)_{\mathbb{R}}=\mathbb{Q}\left(\zeta_{n}\right)_{\mathbb{R}}$. Since $\zeta_{n}+\bar{\zeta}_{n} \notin \mathbb{Q}$, we have $d, n>2$. Moreover, since $\left[\mathbb{Q}\left(\zeta_{k}+\bar{\zeta}_{k}\right): \mathbb{Q}\right]=\frac{\phi(k)}{2}$ if $k>2($ cf. [FT, Theorem $44]$ ), we have $\phi(n)=\phi(d)$. Finally since $d \mid n$, either $d=n$ or $d$ is odd and $2 d=n$. In other words either $n$ divides $m$ or $n$ is even, $\frac{n}{2}$ is odd and $n$ divides $2 m$.

In what follows we let

$$
\Delta(a, b, c)=\left\langle x, y \mid x^{a}, y^{b},(x y)^{c}\right\rangle
$$

denote the $(a, b, c)$ triangle group.

Lemma 8.2. Let $\rho: \Delta(a, b, c) \rightarrow P S L_{2}(\mathbb{C})$ be a homomorphism and suppose that the image of $\rho$ contains an element of order $n<\infty$. Then either $n \in\{1,2,3\}$ or $n$ divides the least common multiple of $a, b, c$.

Proof. There are matrices $A, B, C \in S L_{2}(\mathbb{C})$ whose orders divide $2 a, 2 b, 2 c$, respectively, so that $\rho(x)= \pm A, \rho(y)= \pm B, \rho(x y)= \pm C$. Then $\operatorname{trace}(A)=\zeta_{2 a}^{j}+$ $\bar{\zeta}_{2 a}^{j}, \operatorname{trace}(B)=\zeta_{2 b}^{k}+\bar{\zeta}_{2 b}^{k}$ and trace $(C)=\zeta_{2 c}^{l}+\bar{\zeta}_{2 c}^{l}$ for some integers $j, k, l$. Since the trace of any word in $A, B$ is an integral polynomial in the traces of $A, B$ and $C$ [CS, proof of proposition 1.4.1], such a trace lies in the field $\mathbb{Q}\left(\zeta_{2 a}, \zeta_{2 b}, \zeta_{2 c}\right)=\mathbb{Q}\left(\zeta_{h}\right)$ where $h=2 \operatorname{lcm}(a, b, c)[\mathrm{FT}, \mathrm{VI} .2 .8]$.

Let $W \in S L_{2}(\mathbb{C})$ be a matrix of order $2 n$ whose image $[W]$ in $\operatorname{PSL}(2, \mathbb{C})$ is an element of order $n$ in the image of $\rho$. Then $\operatorname{trace}(W)=\zeta_{2 n}^{m}+\bar{\zeta}_{2 n}^{m}$ for some $m$ relatively prime to $2 n$. Fix a word $w$ so that the element $w(x, y) \in \Delta(a, b, c)$ satisfies $[W]=\rho(w(x, y))=[w(A, B)]$. Then by the previous paragraph we have

$$
\zeta_{2 n}^{m}+\bar{\zeta}_{2 n}^{m}=\operatorname{trace}(W) \in\{ \pm \operatorname{trace}(w(A, B))\} \subset \mathbb{Q}\left(\zeta_{h}\right) .
$$

As $m$ is relatively prime to $2 n$ we have

$$
\zeta_{2 n}+\bar{\zeta}_{2 n} \in \mathbb{Q}\left(\zeta_{2 n}^{m}\right)_{\mathbb{R}}=\mathbb{Q}\left(\zeta_{2 n}^{m}+\bar{\zeta}_{2 n}^{m}\right) \subset \mathbb{Q}\left(\zeta_{h}\right) .
$$

Lemma 8.1 now yields the desired conclusion.

Lemma 8.3. Let $M$ be a simple knot manifold. Fix slopes $\alpha$ and $\beta$ on $\partial M$. Suppose that $M(\beta)$ is a connected sum of two lens spaces whose fundamental groups have orders $p, q \geq 2$, and that $M(\alpha)$ is a Seifert fibered space whose base orbifold has the form $S^{2}(a, b, c)$ where $a, b, c \geq 2$. If $\Delta(\alpha, \beta)>3$, then $\Delta(\alpha, \beta)$ divides lcm $(a, b, c)$. 
Proof. Fix a point on $\partial M$ so that we have homomorphisms $H_{1}(\partial M) \cong \pi_{1}(\partial M) \rightarrow$ $\pi_{1}(M)$. In this way each slope $r$ on $\partial M$ determines an element $\gamma(r)$ of $\pi_{1}(M)$ well defined up to taking an inverse.

There is a curve $X_{0}$ contained in the $P S L_{2}(\mathbb{C})$-character variety of $\pi_{1}(M)$ containing the character of an irreducible representation and consisting of characters $\chi_{\rho}$ of representations $\rho: \pi_{1}(M) \rightarrow P S L_{2}(\mathbb{C})$ which factor through $\pi_{1}(M(\beta)) \cong$ $\mathbb{Z} / p * \mathbb{Z} / q$ BZ, Example 3.2]. For each slope $r$ let $f_{r}: X_{0} \rightarrow \mathbb{C}$ be the regular function $f_{r}\left(\chi_{\rho}\right)=\operatorname{trace}(\rho(\gamma(r)))^{2}-4$. Evidently $f_{\beta}$ is identically zero. We claim that for each $r \neq \beta$ and ideal point $x$ of $X_{0}, f_{r}$ has a pole at $x$. If this were not the case, there would be a closed essential surface $S \subset M$ which remains essential in either $M(\beta)$ or $M(\alpha)$ [BZ, Proposition 4.10]. But $S$ compresses in both $M(\beta)$ and $M(\alpha)$. This is obvious for $M(\beta)$, while if $S$ is essential in $M(\alpha)$, then $S$ is a fiber in some realization of $M(\alpha)$ as a surface bundle over the circle (see eg. Ja VI.34]) and so it is non-separating in $M$. But then $b_{1}(M) \geq 2$, contrary to the fact that $b_{1}(M(\beta))=0$. Thus $S$ compresses in $M(\alpha)$ and therefore $f_{r}$ has a pole at $x$.

Let $r$ be a slope so that $\Delta(r, \beta)=1$. From the previous paragraph there is a character $\chi_{\rho} \in X_{0}$ at which $f_{r}$ takes the value $\left(e^{\pi i / \Delta(\alpha, \beta)}+e^{-\pi i / \Delta(\alpha, \beta)}\right)^{2}$. The representation $\rho$ may be taken to factor through $\pi_{1}(M(\beta))$ and to have a non-diagonalisable image (cf. the method of proof of [CGLS] Lemma 1.5.10]). Since $\Delta(\alpha, \beta)>1$, $\rho(\gamma(r))$ has order $\Delta(\alpha, \beta)$, while by construction $\rho(\gamma(\beta))= \pm I$. It follows that $\rho(\gamma(\alpha))= \pm I$ and therefore $\rho$ factors through a representation $\rho_{1}: \pi_{1}(M(\alpha)) \rightarrow$ $P S L_{2}(\mathbb{C})$. In fact, $\rho$ further factors through $\pi_{1}^{\text {orb }}\left(S^{2}(a, b, c)\right) \cong \Delta(a, b, c)$. To see this, first observe that since $b_{1}(M)=1, \chi_{\rho}$ is a non-trivial character $[\mathrm{B}$, Proposition $2.8]$ and therefore $[\mathrm{BB}$, Lemma 3.1] implies that $\rho$ factors as claimed. In conclusion we have produced a homomorphism $\Delta(a, b, c) \rightarrow P S L_{2}(\mathbb{C})$ which contains an element of order $\Delta(\alpha, \beta)>3$ in its image. Apply Lemma 8.2 to see that $\Delta(\alpha, \beta)$ divides $l c m(a, b, c)$.

Proposition 8.4. Let $M$ be a simple knot manifold and fix slopes $\alpha$ and $\beta$ on $\partial M$. Suppose that $M(\beta)$ is reducible, but not homeomorphic to $S^{1} \times S^{2}$ or $P^{3} \# P^{3}$, and that $M(\alpha)$ is a Seifert fibered manifold. If $\Delta(\alpha, \beta)>3$, then

(i) $M(\beta)$ is a connected sum of two lens spaces.

(ii) $M(\alpha)$ admits a Seifert structure whose base orbifold is the 2-sphere with exactly three exceptional fibers whose orders $a, b, c$ are either a Platonic or hyperbolic triple.

(iii) $\Delta(\alpha, \beta)$ is equal to 4,5 or 6 and divides $\operatorname{lcm}(a, b, c)$.

Proof. We first show that $\Delta(\alpha, \beta) \leq 1$ when $b_{1}(M) \geq 2$. In this case, according to $\mathrm{Ga}$, the slope $\beta$ is the unique degenerating slope for a closed non-separating essential surface $S_{*}$ (which is Thurston norm minimizing in the homology class it represents) of genus larger than 1 in $M$, i.e. $S_{*}$ will remain incompressible in $M(\delta)$ for any slope $\delta$ except for $\delta=\beta$. Hence the irreducible manifold $M(\alpha)$ cannot be very small. If it is Seifert fibered we have $\Delta(\alpha, \beta) \leq 1$ by [BGZ Proposition 5.1].

We may therefore assume that $b_{1}(M)=1$. If $M(\alpha)$ contains an embedded incompressible torus, then $\Delta(\alpha, \beta) \leq 3$ by $[\mathrm{O}$, [Wu. If $M(\alpha)$ is geometrically atoroidal, then it admits a Seifert structure with three or fewer exceptional fibers and whose base orbifold has the 2-sphere for underlying space. When there are no more than two exceptional fibers it is known that $\Delta(\alpha, \beta) \leq 1$ [BZ. Theorem 1.2(1)], while if the base orbifold of $M(\alpha)$ has the form $S^{2}(a, b, c)$ where $a, b, c \geq 2$ it is known that $\Delta(\alpha, \beta) \leq 3$ if $(a, b, c)$ is a Euclidean triple $[\mathrm{B}$, Theorem $\mathrm{C}]$. Thus 
(ii) holds. By CGLS, Theorem 2.0.3] $M(\beta)$ is a connected sum of two lens spaces, so (i) holds. Finally (iii) is a consequence of Corollary 7.4.6 and Lemma 8.3.

Corollary 8.5. Let $M$ be a simple knot manifold and fix slopes $\alpha$ and $\beta$ on $\partial M$. If $M(\beta)$ is reducible, though not $S^{1} \times S^{2}$ or $P^{3} \# P^{3}$, and $M(\alpha)$ is a Seifert fibered space, then $\Delta(\alpha, \beta) \leq 5$ unless perhaps $M(\beta) \cong P^{3} \# L(p, q)$ and $M(\alpha)$ is a small Seifert manifold with base orbifold $S^{2}(a, b, c)$ where $(a, b, c)$ is a hyperbolic triple and 6 divides $l \mathrm{~cm}(a, b, c)$.

Proof. The corollary follows from Theorem 7.4.6, the previous proposition and the fact that $\Delta(\alpha, \beta) \leq 5$ if $(a, b, c)$ is a Platonic triple [BZ, Theorem 1.2(2)].

There is another situation where we can use the same method to sharpen the known distance bounds.

Lemma 8.6. Let $M$ be a simple knot manifold and fix slopes $\alpha$ and $\beta$ on $\partial M$. Suppose that $M(\beta)$ is a Seifert fibered manifold which contains an embedded incompressible torus and that $M(\alpha)$ admits a Seifert fibration whose base orbifold has the form $S^{2}(a, b, c)$, where $a, b, c \geq 2$. If $\Delta(\alpha, \beta)>5$, then $\Delta(\alpha, \beta)$ divides $\operatorname{lcm}(a, b, c)$.

Proof. By [BGZ, Theorems 1.1 and 1.7] we may assume that $b_{1}(M)=1$ and that $M(\beta)$ has base orbifold a Klein bottle, $S^{2}(2,2,2,2)$, or $P^{2}(p, q)$ for some integers $p, q \geq 2$. In each case there is a curve $X_{0} \subset X\left(\pi_{1}(M)\right)$ containing the character of an irreducible representation and consisting of characters of representations $\rho: \pi_{1}(M) \rightarrow P S L_{2}(\mathbb{C})$ which factor through $\pi_{1}(M(\beta))$ [BZ Lemma 8.7]. If for some slope $r \neq \beta$ on $\partial M$ and ideal point $x$ of $X_{0}, f_{r}$ is finite at $x$, then there is a closed, essential surface $S \subset M$ which is incompressible in at least one of $M(\beta)$ and $M(\alpha)$ [BZ, Proposition 4.10]. It was shown in [BZ Claim, page 786] that $S$ compresses in $M(\beta)$, so it must be essential in $M(\alpha)$. But this would imply that $b_{1}(M) \geq 2$, contrary to our assumptions (cf. the proof of Lemma 8.3). Thus for each slope $r \neq \beta$ on $\partial M$, the function $f_{r}$ has a pole at each ideal point $x$ of $X_{0}$. We now proceed as in the last paragraph of the proof of Lemma 8.3 to see that $\Delta(\alpha, \beta)$ divides $l c m(a, b, c)$.

Proposition 8.7. Let $M$ be a simple knot manifold and fix slopes $\alpha$ and $\beta$ on $\partial M$. Suppose that $M(\beta)$ is a Seifert fibered manifold that contains an embedded incompressible torus, but is not homeomorphic to the union of two twisted I-bundles over Klein bottles, and that $M(\alpha)$ is a Seifert fibered manifold. If $\Delta(\alpha, \beta)>5$, then

(i) $M(\beta)$ admits a Seifert fibration over $P^{2}$ with exactly two exceptional fibers whose orders are $p$ and $q$ for some integers $p>q \geq 2$.

(ii) $M(\alpha)$ admits a Seifert fibration over the 2-sphere with exactly three exceptional fibers whose orders a,b,c form either a hyperbolic triple or the Euclidean triple $2,3,6$.

(iii) $\Delta(\alpha, \beta) \in\{6,7,8,9,10\}$ and $\Delta(\alpha, \beta)$ divides $\operatorname{lcm}(a, b, c)$.

Proof. By [BGZ, Theorems 1.1 and 1.7] we may assume that $b_{1}(M)=1$ and that $M(\beta)$ has base orbifold of the form $K$, the Klein bottle, $S^{2}(2,2,2,2)$, or $P^{2}(p, q)$ for some integers $p, q \geq 2$. Since $M(\beta)$ is not the union of two twisted $I$-bundles over the Klein bottle, its base orbifold must be of the form $P^{2}(p, q)$ for some integers $p>q \geq 2$. Thus (i) holds. 
Next observe that if $M(\alpha)$ contains an incompressible torus, then it is shown in Go2] that $\Delta(\alpha, \beta) \leq 5$. Thus $M(\alpha)$ admits a Seifert structure whose base orbifold $\mathcal{B}$ is a 2 -sphere with three or fewer cone points. Theorem 1.5 of [BZ] shows that $\mathcal{B}=S^{2}(a, b, c)$, where $(a, b, c)$ is a Euclidean or hyperbolic triple. The former possibility is ruled out as in the proof of Theorem $\mathrm{C}$ of $[\mathrm{B}]$ unless $a, b, c$ is the Euclidean triple 2,3,6. Finally by $\mathrm{A}$ or $\mathrm{La}, \Delta(\alpha, \beta) \in\{6,7,8,9,10\}$ and the previous lemma shows that it divides $\operatorname{lcm}(a, b, c)$.

\section{REFERENCES}

[A] Agol, Ian, "Bounds on exceptional Dehn filling," Geom. Topol. 4 (2000), 431-449 (electronic). MR2001j:57019

[BB] L. Ben Abdelghani and S. Boyer, "A calculation of the Culler-Shalen seminorms associated to small Seifert Dehn fillings," Proc. Lond. Math. Soc. 83 (2001), 235-256. MF,2002e:57002

[B] S. Boyer, "On the local structure of $S L_{2}(\mathbb{C})$-character varieties at reducible characters," Top. Appl. 121 (2002), 383-413. MR2003e:57025

[BGZ] S. Boyer, C. McA. Gordon, and X. Zhang, "Dehn fillings of large hyperbolic 3-manifolds", J. Differential Geom. 58 (2001), no. 2, 263-308. MR2003j:57025

[BZ] S. Boyer, and X. Zhang, "On Culler-Shalen seminorms and Dehn filling," Ann. of Math. (2) 148 (1998), 737-801. MR2000d:57028

[CGLS] M. Culler, C. McA. Gordon, J. Luecke, and P. B. Shalen, "Dehn surgery on knots," Ann. of Math. (2) 125 (1987), 237-300. MR88a:57026

$[\mathrm{CM}] \quad$ C. Cao and R. Meyerhoff, "The orientable cusped hyperbolic 3-manifolds of minimum volume," Invent. Math. 146 (2001), 451-478. MR2002i:57016

[CL] D. Cooper and D. Long, "Virtually Haken Dehn-filling." J. Differential G eom. 52 (1999), 173-187. MR2001a:57025

[CS] M. Culler and P. B. Shalen, "Varieties of group representations and splittings of 3manifolds," Ann. of Math. (2) 117 (1983), 109-146. MR84k:57005

[E] D. B. A. Epstein, "Curves on 2-manifolds and isotopies," Acta Math. 115 (1966), 83-107. MR.35:4938

[FT] A. Frolich and M. Taylor, Algebraic Number Theory, Cambridge studies in advanced mathematics 27, Cambridge University Press, 1991. MR 94d:11078

[Ga] D. Gabai, "Foliations and the topology of 3-manifolds II," J. Diff. Geom. 26 (1987) 461-478. MR89a:57014a

[Go1] C. MacA. Gordon, "Dehn filling: a survey." Knot theory (Warsaw, 1995), Banach Center Publ., 42, Polish Acad. Sci., Warsaw, 1998, 129-144. MR99e:57028

[Go2] C. MacA. Gordon, "Toroidal Dehn surgeries on knots in lens spaces." Math. Proc. Cambridge Philos. Soc. 125 (1999), 433-440. MF.99h:57006

[GLi] C. McA. Gordon and Litherland, "Incompressible planar surfaces in 3-manifolds" Topology Appl. 18 (1984), 121-144. MR 86e:57013

[GL] C. McA. Gordon and J. Luecke, "Reducible manifolds and Dehn surgery," Topology 35 (1996), 385-409. MR97b:57013

[Ja] W. Jaco, Lectures on three-manifold topology, CBMS Regional Conference Series in Mathematics, no. 43, American Mathematical Society, Providence, R.I., 1980. MF 81k:57009

[Jo] K. Johannson, Homotopy equivalences of 3-manifolds with boundaries, Lecture Notes in Mathematics, no. 761. Springer, Berlin, 1979. MR82c:57005

[JS] W. Jaco and P. B. Shalen, "Seifert fibered spaces in 3-manifolds," Mem. Amer. Math. Soc. 21 (1979), no. 220. MR81c:57010

[La] M. Lackenby, "Hyperbolic Dehn surgery," Invent. Math. 140 (2000), 243-282. MF 2001m:57003

[Li] T. Li, "Immersed essential surfaces in hyperbolic 3-manifolds," Comm. An al. Geom. 10 (2002), 275-290. MR2003e:57028

[O] S. Oh, "Reducible and toroidal 3-manifolds obtained by Dehn fillings," Topology Appl. 75 (1997), 93-104. MR 98a:57027

[T] I. Torisu, Boundary slopes for knots. Osaka J. Math. 33 (1996), no. 1, 47-55. MF.97h:57024 
[Wa] F. Waldhausen, "On irreducible 3-manifolds which are sufficiently large," Ann. of Math. (2) 87 (1968), 56-88. MR36:7146

[Wu $\mathrm{Y}$. Wu, "Dehn fillings producing reducible manifolds and toroidal manifolds," Topology 37 (1998), 95-108. MR 98j:57033

Département de Mathématiques, Université du Québec, Montréal, P. O. Box 8888, Postal Station Centre-ville Montréal, Québec, Canada H3C 3P8

E-mail address: boyer@math.uqam.ca

Department of Mathematics, Statistics and Computer Science (M/C 249), University of Illinois at Chicago, 851 South Morgan Street, Chicago, Illinois 60607-7045

E-mail address: culler@math.uic.edu

Department of Mathematics, Statistics and Computer Science (M/C 249), University of Illinois at Chicago, 851 South Morgan Street, Chicago, Illinois 60607-7045

E-mail address: shalen@math.uic.edu

Department of Mathematics, SUNy at Buffalo, Buffalo, New York 14260-2900

E-mail address: xinzhang@math.buffalo.edu 